\title{
Synthesis and inverse virtual screening of new bi-cyclic structures towards cancer-relevant cellular targets
}

\author{
Letizia Crocetti ${ }^{1}$ - Giuseppe Floresta ${ }^{2,3} \cdot$ Shabnam Nazir $^{2} \cdot$ Claudia Vergelli $^{1} \cdot$ Amrit Bhogal $^{2} \cdot$ Claudio Biancalani $^{1}$. \\ Nicoletta Cesari ${ }^{1} \cdot$ Maria Paola Giovannoni ${ }^{1} \cdot$ Agostino Cilibrizzi $^{2}$ (I)
}

Received: 6 January 2022 / Accepted: 25 January 2022 / Published online: 11 February 2022

(c) The Author(s) 2022

\begin{abstract}
We report here synthetic approaches to access new classes of small molecules based on three heterocyclic scaffolds, i.e. 3,7-dihydropyrimido[4,5-d]pyridazine-4,8-dione, 1,8-naphthyridin-4(1H)-one and 4H-pyrido[1,2-a]pyrimidin-4-one. The bi-cyclic structure 3,7-dihydropyrimido[4,5-d]pyridazine-4,8-dione is a new heterocycle, described here for the first time. In silico methodologies of inverse virtual screening have been used to preliminary analyse the molecules, in order to explore their potential as hits for chemical biology investigations. Our computational study has been conducted with 43 synthetically accessible small molecules towards 31 cellular proteins involved in cancer pathogenesis. Binding energies were quantified using molecular docking calculations, allowing to define the relative affinities of the ligands for the cellular targets. Through this methodology, 16 proteins displayed effective interactions with distinct small molecules within the matrix. In addition, 23 ligands have demonstrated high affinity for at least one cellular protein, using as reference the co-crystallised ligand in the X-ray structure. The evaluation of ADME and drug score for selected hits also highlights that these new molecular series can serve as sources of lead candidates for further structure optimisation and biological studies.
\end{abstract}

Keywords Inverse virtual screening $\cdot$ Heterocycles $\cdot$ Scaffold diversity $\cdot$ Bi-cyclic scaffold $\cdot$ ADME assessment

\section{Introduction}

Heterocyclic chemistry is a key source of compounds for drug discovery due to the capability of the resulting molecules to imitate the structure of endogenous ligands and bind to a variety of biological targets $[1,2]$. In medicinal chemistry, heterocyclic structures offer the advantage of generating molecular libraries based on a specific core scaffold, permitting screening tests and structure-activity relationship (SAR) studies for the targets of interest [3]. In particular, fused heterocycles can be generated with nearly boundless

Agostino Cilibrizzi

agostino.cilibrizzi@kcl.ac.uk

1 Department of NEUROFARBA, University of Florence, Via Ugo Schiff 6, Sesto Fiorentino, FI 50019, Italy

2 Institute of Pharmaceutical Science, King's College London, Stamford Street, London SE1 9NH, UK

3 Department of Analytical, Environmental and Forensic Sciences, King's College London, 150 Stamford Street, London SE1 9NH, UK combinations, to result into novel bi- or polycyclic frameworks with diverse physico-chemical and biological properties. Overall, the combination of rings leads to rigid and sterically well-defined structures, ensuring a high degree of functional specialisation, as well as the capability to arrange substituents in three-dimensional space as required by nature for interactions with biological targets [4]. From cancer therapy to the treatment of infectious, parasitic and metabolic illnesses, heterocyclic drugs are often used to interfere with the actions of hormones, the neuronal transmission or the activity of enzymes, to name a few.

Scaffold diversity (i.e. variation of the nature of core scaffolds), appendage diversity (i.e. variation in structural moieties around a common scaffold), functional group diversity (i.e. variation of the functional groups present in the molecules) and stereochemical diversity (i.e. variation in the three-dimensional orientation of macromolecule-interacting residues) are determinant for late state chemical functionalisation in drug discovery [5]. Undoubtedly, the more diverse a molecule is, the more likely it can interact with a specific biological macromolecule in a distinctive way [6-9]. In particular, scaffold diversity demonstrates a key element 
to reach increased structural variation and investigate new areas of the chemical space for biological investigations [10]. In this regard, it has been reported that the overall shape diversity of small molecules is formally subordinate to the nature of the particular molecular scaffold, with the peripheral substituents having a lower impact [7]. Therefore, the exploitation of scaffold diversity represents a valid strategy to implement the whole structural diversity of small molecule libraries [10-12].

In the context of exploring scaffold diversity in biology and medicine, heterocycles can lead to increase molecular functionalisation, due to their potential in terms of structural isomerism. This can enhance the overall diversity within libraries, as well as the possibility of identifying hit candidates. Additionally, heterocycles containing nitrogen, oxygen or sulphur that can form hydrogen bonds with functional groups within the protein binding site offer wide opportunities to carry out drug optimisation strategies, aimed at tuning the affinity for a particular target, as well as the solubility, lipophilicity, polarity and the overall drug-like properties.

Computational methods such as structure-based and ligand-based screening are widely used in various drug discovery contexts, spanning from hit identification to lead optimisation stages $[13,14]$. However, these virtual screening approaches are somehow limited to the analysis of only one target at a time $[15,16]$. In contrast, inverse virtual screening (iVS) is a computational technique where libraries of compounds are simultaneously tested towards multiple potential targets, in order to identify hits with pharmacological activity [17-19]. Overall, iVS allows for a quicker analysis of target identification, potential side effects, toxicity, drug repositioning, receptor design and multitarget therapy.

Herein, we report the synthesis and iVS analysis of 43 new heterocyclic small-molecules towards a scaffold-guided structural diversity approach, to identify new hits for biological studies in the field of cancer therapy. Specifically, 31 cellular proteins that are responsible for promoting growth, proliferation, differentiation and metabolism in cancer cells [20, 21] were screened against the 43 novel heterocyclic small molecules based on pyrimido[4,5-d]pyridazine-4,8-dione, 1,8-naphthyridin-4-one and pyrido(1,2-a)pyrimidine scaffolds (Fig. 1). The pyrimido[4,5-d]pyridazine-4,8-dione is a new bi-cyclic structure, whose synthesis was not reported in the literature to date. This study indicates that most of the compounds within the library have potential to interact with the examined targets, representing a valid starting point to drive biological evaluation in a rapid and cost-effective fashion. The molecules were also screened via physiologically based pharmacokinetic (PBPK) analysis, to further assess permeability and absorption features as drug candidates.
Fig. 1 Schematic representation of the two-step design<smiles>O=c1cc[nH]c2ccccc12</smiles>

\section{Search for bioisosteric replacements}<smiles>Cc1nc2c(=O)[nH]nc(C)c2c(=O)[nH]1</smiles><smiles>Cc1ccc2c(=O)c(C)c[nH]c2n1</smiles><smiles>CCCCCCc1cnc2ccccn2c1=O</smiles>

\section{Ligand growing experiments}<smiles>[R]c1nn([R8])c(=O)c2nc([R])n([R3])c(=O)c12</smiles>

pyrimido[4,5-d]pyridazines<smiles>[R]C(=O)c1cn([R1])c2nc([R2])ccc2c1=O</smiles>

1,8-naphtyridin-4-ones<smiles>[R]N([R])C(=O)c1cnc2cccc(C)n2c1=O</smiles>

pyrido[1,2-a]pyrimidines 


\section{Materials and methods}

\section{General remarks}

Reagents and starting materials were obtained from commercial sources. Extracts were dried over $\mathrm{Na}_{2} \mathrm{SO}_{4}$ and the solvents were removed under reduced pressure. All reactions were monitored by thin layer chromatography (TLC) using commercial plates (Merck) pre-coated with silica gel $60 \mathrm{~F}-254$. Visualisation was performed by UV fluorescence $(\lambda \max =254 \mathrm{~nm})$ or by staining with iodine or potassium permanganate. Chromatographic separations were performed on silica gel columns by gravity (Kieselgel 40, 0.063-0.200 mm; Merck) or flash chromatography (Kieselgel 40, 0.040-0.063 mm; Merck). Yields refer to chromatographically and spectroscopically pure compounds, unless otherwise stated. When reactions were performed in anhydrous conditions, the mixtures were maintained under nitrogen atmosphere. Compounds were named following IUPAC rules as applied by BeilsteinInstitut AutoNom 2000 (4.01.305) or CA Index Name. The identity and purity of intermediates and final compounds were ascertained through TLC chromatography, NMR and mass spectrometry. ${ }^{1} \mathrm{H}$ NMR spectra were recorded on the Avance 400 instrument (Bruker Biospin Version 002 with SGU). Chemical shifts ( $\delta$ ) are reported in ppm to the nearest $0.01 \mathrm{ppm}$, using the solvent as internal standard. Coupling constants ( $J$ values) are given in $\mathrm{Hz}$ and were calculated using 'TopSpin 1.3' software rounded to the nearest $0.1 \mathrm{~Hz}$. Data are reported as follows: chemical shift, multiplicity (exch, exchange; br, broad; s, singlet; d, doublet; t, triplet; q, quartet; quin, quintet; sext, sextet; sept, septet; m, multiplet; or as a combination of these (e.g. dd, dt etc.)), integration, assignment and coupling constant(s). All melting points were determined on a microscope hot stage Büchi apparatus and are uncorrected.

\section{Chemistry}

General procedure for compounds 5c, 5e Hydrazine hydrate $(3 \mathrm{mmol})$ was added to a cooled $\left(0{ }^{\circ} \mathrm{C}\right)$ and stirred suspension of commercially available isoxazole derivatives $\mathbf{4 c , e}(2 \mathrm{mmol})$ and PPA $(50 \mathrm{mmol})$ in ethanol $(5 \mathrm{~mL})$, and the mixture was heated under stirring for $2-5 \mathrm{~h}$ at $80-90{ }^{\circ} \mathrm{C}$. After dilution with cold water $(20-30 \mathrm{~mL})$, pure compounds $\mathbf{5 c}$ and $\mathbf{5 e}$ were recovered by filtration under vacuum.

- 4-(3-Chlorophenyl)isoxazolo[3,4-d]pyridazin-7(6H)one $(5 \mathrm{c})$

Yield $=96 \%,(475 \mathrm{mg}) ; \mathrm{mp}=241-244{ }^{\circ} \mathrm{C}(\mathrm{EtOH})$. ${ }^{1} \mathrm{H}-\mathrm{NMR}\left(400 \mathrm{MHz}\right.$, DMSO-d $\left.{ }_{6}\right) \delta$ 7.55-7.65 (m, 2H, Ar), $7.90(\mathrm{~d}, 2 \mathrm{H}, \mathrm{Ar}, J=8.0 \mathrm{~Hz}), 10.35(\mathrm{~s}, 1 \mathrm{H}$, isox- azole), 12.90 (exch br s, 1H, NH). Anal. Calcd. for $\mathrm{C}_{11} \mathrm{H}_{6} \mathrm{ClN}_{3} \mathrm{O}_{2}$ : C 53.35, H 2.44, N 16.97. Found: C 53.21, H 2.44, N 16.92.

- 4-Pyridin-3-yl-isoxazolo[3,4-d]pyridazin-7(6H)-one (5e)

Yield $=69 \%,(463 \mathrm{mg}) ; \mathrm{mp}=227-228^{\circ} \mathrm{C}(\mathrm{EtOH})$. ${ }^{1} \mathrm{H}-\mathrm{NMR}\left(400 \mathrm{MHz}, \mathrm{DMSO}-\mathrm{d}_{6}\right) \delta$ 7.55-7.65 (m, 1H, $\mathrm{Ar}), 8.25(\mathrm{~d}, 1 \mathrm{H}, \mathrm{Ar}, J=8.4 \mathrm{~Hz}), 8.75(\mathrm{~d}, 1 \mathrm{H}, \mathrm{Ar}, J$ $=8.2 \mathrm{~Hz}), 9.05(\mathrm{~s}, 1 \mathrm{H}, \mathrm{Ar}), 10.35(\mathrm{~s}, 1 \mathrm{H}$, isoxazole $)$, 12.90 (exch br s, $1 \mathrm{H}, \mathrm{NH}$ ). Anal. Calcd. for $\mathrm{C}_{10} \mathrm{H}_{6} \mathrm{~N}_{4} \mathrm{O}_{2}$ : C 56.08, H 2.82, N 26.16. Found: C 56.23, H 2.81, N 26.23 .

General procedure for compounds 6a-e A suspension of isoxazolo pyridazinones 5a-e $(0.7-1.2 \mathrm{mmol})$ in $33 \%$ $\mathrm{NH}_{4} \mathrm{OH}(2.0-2.5 \mathrm{~mL})$ was stirred at $60{ }^{\circ} \mathrm{C}$ for $1-2 \mathrm{~h}$. After cooling, the precipitate was recovered by filtration under vacuum to give the pure intermediates 6a-d. For compound $\mathbf{6 e}$, the mixture was diluted with water, the suspension was extracted with $\mathrm{CH}_{2} \mathrm{Cl}_{2}(3 \times 15 \mathrm{~mL})$ and the solvent was evaporated in vacuo to afford the compound.

- 5-Amino-6-oxo-3-phenyl-1,6-dihydropyridazine4-carboxylic acid amide (6a)

Yield $=46 \%(74 \mathrm{mg}) ; \mathrm{mp} \geq 300{ }^{\circ} \mathrm{C}(\mathrm{EtOH}) .{ }^{1} \mathrm{H}-\mathrm{NMR}$ $\left(400 \mathrm{MHz}, \mathrm{DMSO}_{-} \mathrm{d}_{6}\right) \delta 6.40$ (exch br s, $\left.2 \mathrm{H}, \mathrm{NH}_{2}\right), 7.30$ 7.45 (m, 5H, Ar), 7.45-7.50 (exch br s, $2 \mathrm{H}, \mathrm{CONH}_{2}$ ), 12.80 (exch br s, $1 \mathrm{H}, \mathrm{NH}$ ). Anal. Calcd. for $\mathrm{C}_{11} \mathrm{H}_{10} \mathrm{~N}_{4} \mathrm{O}_{2}$ : C 57.39, H 4.38, N 24.34. Found: C 57.55, H 4.37, N 24.26.

- 5-Amino-3-(3-fluorophenyl)-6-oxo-1,6-dihydropyridazine4-carboxylic acid amide (6b)

Yield $=50 \%(119 \mathrm{mg}) ; \mathrm{mp}=285-287^{\circ} \mathrm{C}(\mathrm{EtOH}) .{ }^{1} \mathrm{H}-$ NMR $\left(400 \mathrm{MHz}\right.$, DMSO-d $\left.{ }_{6}\right) \delta 6.45$ (exch br s, $2 \mathrm{H}, \mathrm{NH}_{2}$ ), 7.20-7.30 (m, 2H, Ar), 7.35 (d, 1H, Ar, J=7.8 Hz), 7.45 (m, 1H, Ar), 7.50 (exch br s, 2H, $\mathrm{CONH}_{2}$ ), 12.10 (exch br s, $1 \mathrm{H}, \mathrm{NH}$ ). Anal. Calcd. for $\mathrm{C}_{11} \mathrm{H}_{9} \mathrm{FN}_{4} \mathrm{O}: \mathrm{C} 53.23, \mathrm{H}$ 3.65, N 22.57. Found: C 53.06, H 3.66, N 22.62.

- 5-Amino-3-(3-chlorophenyl)-6-oxo-1,6-dihydropyridazine4-carboxylic acid amide (6c)

Yield $=63 \%(183 \mathrm{mg}) ; \mathrm{mp}=257-260{ }^{\circ} \mathrm{C}(\mathrm{EtOH})$. ${ }^{1} \mathrm{H}-\mathrm{NMR}$ (400 MHz, DMSO-d 6 ) $\delta 6.45$ (exch br s, $2 \mathrm{H}, \mathrm{NH}_{2}$ ), 7.40-7.50 (m, 4H, Ar), 7.55 (exch br s, 2H, $\mathrm{CONH}_{2}$ ), 11.90 (exch br s, $1 \mathrm{H}, \mathrm{NH}$ ). Anal. Calcd. for $\mathrm{C}_{11} \mathrm{H}_{9} \mathrm{ClN}_{4} \mathrm{O}_{2}$ : C 49.92, H 3.43, N 21.17. Found: C 49.79, H 3.42, N 21.25.

- 5-Amino-6-oxo-3-m-tolyl-1,6-dihydropyridazine-4-carboxylic acid amide $(\boldsymbol{6 d})$

Yield $=60 \%(144 \mathrm{mg}) ; \mathrm{mp}=263-265^{\circ} \mathrm{C}(\mathrm{EtOH})$. ${ }^{1} \mathrm{H}-\mathrm{NMR}\left(400 \mathrm{MHz}\right.$, DMSO-d 6 ) $\delta 2.30\left(\mathrm{~s}, 3 \mathrm{H}, \mathrm{CH}_{3}\right)$, 6.40 (exch br s, 2H, $\mathrm{NH}_{2}$ ), 7.15-7.35 (m, 4H, Ar), 7.40 (exch br s, $2 \mathrm{H}, \mathrm{CONH}_{2}$ ), 12.80 (exch br s, $1 \mathrm{H}$, $\mathrm{NH})$. Anal. Calcd. for $\mathrm{C}_{12} \mathrm{H}_{12} \mathrm{~N}_{4} \mathrm{O}_{2}: \mathrm{C} 59.01, \mathrm{H} 4.95, \mathrm{~N}$ 22.94. Found: C 59.18, H 4.94, N 22.89. 
- 5-Amino-6-oxo-3-pyridin-3-yl-1,6-dihydropyridazine4-carboxylic acid amide (6e)

Yield $=50 \%(139 \mathrm{mg}) ; \mathrm{mp}=244-247{ }^{\circ} \mathrm{C}(\mathrm{EtOH})$. ${ }^{1} \mathrm{H}-\mathrm{NMR}\left(400 \mathrm{MHz}, \mathrm{DMSO}-\mathrm{d}_{6}\right) \delta 6.55$ (exch br s, $2 \mathrm{H}$, $\mathrm{NH}_{2}$ ), 7.40 (m, 1H, Ar), 7.50 (exch br s, $1 \mathrm{H}, \mathrm{CONH}_{2}$ ), 7.55 (exch br s, $\left.1 \mathrm{H}, \mathrm{CONH}_{2}\right), 7.85(\mathrm{~m}, 1 \mathrm{H}, \mathrm{Ar}), 8.55$ (m, 1H, Ar), 8.65 (s, 1H, Ar), 12.95 (exch br s, 1H, $\mathrm{NH})$. Anal. Calcd. for $\mathrm{C}_{10} \mathrm{H}_{9} \mathrm{~N}_{5} \mathrm{O}_{2}: \mathrm{C} 51.95, \mathrm{H} 3.92, \mathrm{~N}$ 30.29. Found: C 51.82, H 3.92, N 30.37.

\section{General procedure for compounds $7 \mathbf{b}-\mathbf{d}$ and $7 \mathbf{~ g - k} A$} mixture of the suitable 5-amino-1,6-dihydropyridazine4-carboxylic acid amide derivatives $\mathbf{6 a}, \mathbf{6 c - e}(0.5 \mathrm{mmol})$, $\mathrm{K}_{2} \mathrm{CO}_{3}(1 \mathrm{mmol})$ and the appropriate alkyl(aryl) halide $(0.75-1.25 \mathrm{mmol})$ was stirred in anhydrous DMF $(1-2 \mathrm{~mL})$ at $80{ }^{\circ} \mathrm{C}$ for $0.5-3 \mathrm{~h}$. For compound $7 \mathbf{j}$, the reaction was carried out at $100{ }^{\circ} \mathrm{C}$ for $7 \mathrm{~h}$. After cooling, the precipitate was recovered by filtration under vacuum to give the pure intermediates $\mathbf{7 b}-\mathbf{d}, \mathbf{7} \mathbf{g}$-i. For compounds $\mathbf{7} \mathbf{j}, \mathbf{k}$, the mixture was diluted with water, the suspension was extracted with $\mathrm{CH}_{2} \mathrm{Cl}_{2}(3 \times 15 \mathrm{~mL})$, and the solvent was evaporated in vacuo to afford the crude compounds. Subsequently, $\mathbf{7 j}, \mathbf{k}$ were purified by column chromatography using $\mathrm{CHCl}_{3}$ / $\mathrm{MeOH}$ 9:1 as eluent.

- 5-Amino-1-(4-nitrobenzyl)-6-oxo-3-phenyl-1,6dihydropyridazine-4-carboxylic acid amide $(7 \boldsymbol{b})$

Yield $=95 \%(173 \mathrm{mg}) ; \mathrm{mp}=213-217{ }^{\circ} \mathrm{C}(\mathrm{EtOH})$. ${ }^{1} \mathrm{H}-\mathrm{NMR}\left(400 \mathrm{MHz}, \mathrm{CDCl}_{3}\right) \delta 4.95$ (exch br s, $1 \mathrm{H}$, $\mathrm{CONH}_{2}$ ), 5.35 (exch br s, $\left.1 \mathrm{H}, \mathrm{CONH}_{2}\right), 5.40(\mathrm{~s}, 2 \mathrm{H}$, $\mathrm{CH}_{2}$ ), 7.50 (s, $\left.5 \mathrm{H}, \mathrm{Ar}\right), 7.60$ (d, $2 \mathrm{H}, \mathrm{Ar}, J=8.2 \mathrm{~Hz}$ ), 8.20 (d, $2 \mathrm{H}, \mathrm{Ar}, J=8.1 \mathrm{~Hz}$ ), 8.40 (exch br s, $2 \mathrm{H}, \mathrm{NH}_{2}$ ). Anal. Calcd. for $\mathrm{C}_{18} \mathrm{H}_{15} \mathrm{~N}_{5} \mathrm{O}_{4}$ : C 59.18, H 4.14, N 19.17. Found: C 59.35, H 4.15, N 19.11.

- 4-(5-Amino-4-carbamoyl-6-oxo-3-phenyl-6H-pyridazin1-ylmethyl)-benzoic acid ethyl ester (7c)

Yield $=97 \%(190 \mathrm{mg}) ; \mathrm{mp}=162-164{ }^{\circ} \mathrm{C}(\mathrm{EtOH})$. ${ }^{1} \mathrm{H}-\mathrm{NMR}\left(400 \mathrm{MHz}, \mathrm{CDCl}_{3}\right) \delta 1.40\left(\mathrm{t}, 3 \mathrm{H}, \mathrm{CH}_{2} \mathrm{CH}_{3}\right.$, $J=7.2 \mathrm{~Hz}), 4.35\left(\mathrm{q}, 2 \mathrm{H}, \mathrm{CH}_{2} \mathrm{CH}_{3}, J=7.2 \mathrm{~Hz}\right), 4.95$ (exch br s, $1 \mathrm{H}, \mathrm{CONH}_{2}$ ), 5.35 (exch br s, $1 \mathrm{H}, \mathrm{CONH}_{2}$ ), $5.40\left(\mathrm{~s}, 2 \mathrm{H}, \mathrm{CH}_{2}\right), 7.50-7.60(\mathrm{~m}, 7 \mathrm{H}, \mathrm{Ar}), 8.05(\mathrm{~d}$, $2 \mathrm{H}, \mathrm{Ar}$ ), 8.10 (exch br s, $2 \mathrm{H}, \mathrm{NH}_{2}$ ). Anal. Calcd. for $\mathrm{C}_{21} \mathrm{H}_{20} \mathrm{~N}_{4} \mathrm{O}_{4}$ : C 64.28, H 5.14, N 14.28. Found: C 64.11, H 5.16, N 14.24.

- 5-Amino-1-(4-hydroxymethylbenzyl)-6-oxo-3-phenyl1,6-dihydropyridazine-4-carboxylic acid amide (7d)

Yield $=77 \%(135 \mathrm{mg}) ; \mathrm{mp}=193-198{ }^{\circ} \mathrm{C}(\mathrm{EtOH})$. ${ }^{1} \mathrm{H}-\mathrm{NMR}\left(400 \mathrm{MHz}, \mathrm{DMSO}-\mathrm{d}_{6}\right) \delta 4.45\left(\mathrm{~d}, 2 \mathrm{H}, \mathrm{CH}_{2} \mathrm{OH}\right.$, $J=5.6 \mathrm{~Hz}$ ), 5.15 (exch br t, $1 \mathrm{H}, \mathrm{CH}_{2} \mathrm{OH}, J=5.6 \mathrm{~Hz}$ ), $5.25\left(\mathrm{~s}, 2 \mathrm{H}, \mathrm{NCH}_{2}\right), 6.55$ (exch br s, $2 \mathrm{H}, \mathrm{CONH}_{2}$ ), 7.257.50 (m, 9H, Ar), 7.65 (exch br s, $2 \mathrm{H}, \mathrm{NH}_{2}$ ). Anal. Calcd. for $\mathrm{C}_{19} \mathrm{H}_{18} \mathrm{~N}_{4} \mathrm{O}_{3}$ : C 65.13, H 5.18, N 15.99. Found: $\mathrm{C}$ 65.30, H 5.17, N 15.94.
- 5-Amino-3-(3-chlorophenyl)-1-cyclopropylmethyl-6-oxo1,6-dihydropyridazine-4-carboxylic acid amide $(7 \mathrm{~g})$

Yield $=60 \%(96 \mathrm{mg}) ; \mathrm{mp}=187-189{ }^{\circ} \mathrm{C}(\mathrm{EtOH}) .{ }^{1} \mathrm{H}-$ NMR (400 MHz, $\left.\mathrm{CDCl}_{3}\right) \delta 0.45-0.60\left(\mathrm{~m}, 4 \mathrm{H}, \mathrm{cC}_{3} \mathrm{H}_{5}\right)$, 1.35-1.45 (m, $\left.1 \mathrm{H}, \mathrm{cC}_{3} \mathrm{H}_{5}\right), 4.05\left(\mathrm{~d}, 2 \mathrm{H}, \mathrm{CH}_{2} \mathrm{cC}_{3} \mathrm{H}_{5}\right.$, $J=7.2 \mathrm{~Hz}$ ), 5.00 (exch br s, $1 \mathrm{H}, \mathrm{CONH}_{2}$ ), 5.40 (exch br s, $\left.1 \mathrm{H}, \mathrm{CONH}_{2}\right), 7.40-7.50(\mathrm{~m}, 3 \mathrm{H}, \mathrm{Ar}), 7.60(\mathrm{~s}$, $1 \mathrm{H}, \mathrm{Ar}$ ), 7.85 (exch br s, $2 \mathrm{H}, \mathrm{NH}_{2}$ ). Anal. Calcd. for $\mathrm{C}_{15} \mathrm{H}_{15} \mathrm{ClN}_{4} \mathrm{O}_{2}$ : C 56.52, $\mathrm{H} 4.74, \mathrm{~N}$ 17.58. Found: $\mathrm{C}$ 56.33, H 4.75, N 17.62 .

- 5-Amino-3-(3-chlorophenyl)-1-cyclohexylmethyl-6-oxo1,6-dihydropyridazine-4-carboxylic acid amide $(7 \mathrm{~h})$

Yield $=60 \%(108 \mathrm{mg}) ; \mathrm{mp}=186-188^{\circ} \mathrm{C}(\mathrm{EtOH}) .{ }^{1} \mathrm{H}-$ NMR (400 MHz, $\left.\mathrm{CDCl}_{3}\right) \delta 1.05-1.30\left(\mathrm{~m}, 5 \mathrm{H}, \mathrm{cC}_{6} \mathrm{H}_{11}\right)$, $1.60-1.80\left(\mathrm{~m}, 5 \mathrm{H}, \mathrm{cC}_{6} \mathrm{H}_{11}\right), 2.00\left(\mathrm{~m}, 1 \mathrm{H}, \mathrm{cC}_{6} \mathrm{H}_{11}\right), 4.05$ (d, $2 \mathrm{H}, \mathrm{CH}_{2}, J=7.2 \mathrm{~Hz}$ ), 4.95 (exch br s, $1 \mathrm{H}, \mathrm{CONH}_{2}$ ), 5.45 (exch br s, $1 \mathrm{H}, \mathrm{CONH}_{2}$ ), 7.40-7.50 (m, 3H, Ar), 7.60 (s, $1 \mathrm{H}, \mathrm{Ar}), 8.10$ (exch br s, $2 \mathrm{H}, \mathrm{NH}_{2}$ ). Anal. Calcd. for $\mathrm{C}_{18} \mathrm{H}_{21} \mathrm{ClN}_{4} \mathrm{O}_{2}$ : C 59.91, H 5.87, N 15.53. Found: $\mathrm{C}$ 59.75, H 5.85, N 15.57.

- 5-Amino-3-(3-chlorophenyl)-1-cyclohexyl-6-oxo1,6-dihydropyridazine-4-carboxylic acid amide (7i)

Yield $=30 \%(52 \mathrm{mg}) ; \mathrm{mp}=244-245{ }^{\circ} \mathrm{C}(\mathrm{EtOH})$. ${ }^{1} \mathrm{H}-\mathrm{NMR}\left(400 \mathrm{MHz}\right.$, DMSO-d $\left.{ }_{6}\right) \delta 1.10-1.25(\mathrm{~m}, 1 \mathrm{H}$, $\left.\mathrm{cC}_{6} \mathrm{H}_{11}\right), 1.35-1.45\left(\mathrm{~m}, 2 \mathrm{H}, \mathrm{cC}_{6} \mathrm{H}_{11}\right), 1.60-1.90(\mathrm{~m}$, $\left.7 \mathrm{H}, \mathrm{cC}_{6} \mathrm{H}_{11}\right), 4.80\left(\mathrm{~m}, 1 \mathrm{H}, \mathrm{cC}_{6} \mathrm{H}_{11}\right), 6.50$ (exch br s, $2 \mathrm{H}$, $\mathrm{CONH}_{2}$ ), 7.40-7.50 (m, 4H, Ar), 7.55-7.65 (exch br s, $2 \mathrm{H}, \mathrm{NH}_{2}$ ). Anal. Calcd. for $\mathrm{C}_{17} \mathrm{H}_{19} \mathrm{ClN}_{4} \mathrm{O}_{2}$ : C 58.87, $\mathrm{H}$ 5.52, N 16.16. Found: C 58.71, H 5.51, N 16.21.

- 5-Amino-1-cyclohexylmethyl-6-oxo-3-m-tolyl-1,6dihydropyridazine-4-carboxylic acid amide (7j)

Yield $=66 \%(112 \mathrm{mg}) ; \mathrm{mp}=166-167{ }^{\circ} \mathrm{C}(\mathrm{EtOH})$. ${ }^{1} \mathrm{H}-\mathrm{NMR}\left(400 \mathrm{MHz}\right.$, DMSO-d $\left.{ }_{6}\right) \delta 0.95-1.30(\mathrm{~m}, 5 \mathrm{H}$, $\left.\mathrm{cC}_{6} \mathrm{H}_{11}\right), 1.55-1.75\left(\mathrm{~m}, 5 \mathrm{H}, \mathrm{cC}_{6} \mathrm{H}_{11}\right), 1.90(\mathrm{~m}, 1 \mathrm{H}$, $\left.\mathrm{cC}_{6} \mathrm{H}_{11}\right), 2.35\left(\mathrm{~s}, 1 \mathrm{H}, \mathrm{CH}_{3}\right), 3.95\left(\mathrm{~d}, 2 \mathrm{H}, \mathrm{CH}_{2} \mathrm{cC}_{6} \mathrm{H}_{11}\right.$, $J=7.2 \mathrm{~Hz}$ ), 6.45 (exch br s, 2H, $\mathrm{CONH}_{2}$ ), 7.15-7.35 (m, $4 \mathrm{H}, \mathrm{Ar}$ ), 7.35-7.45 (exch br s, $2 \mathrm{H}, \mathrm{NH}_{2}$ ). Anal. Calcd. for $\mathrm{C}_{19} \mathrm{H}_{24} \mathrm{~N}_{4} \mathrm{O}_{2}$ : C 67.04, H 7.11, N 16.46. Found: C 67.22, $\mathrm{H} 7.13, \mathrm{~N} 16.42$.

- 5-Amino-1-cyclohexylmethyl-6-oxo-3-pyridin-3-yl1,6-dihydropyridazine-4-carboxylic acid amide (7 k)

Yield $=28 \%(112 \mathrm{mg}) ; \mathrm{mp}=233-236{ }^{\circ} \mathrm{C}(\mathrm{EtOH})$. ${ }^{1} \mathrm{H}-\mathrm{NMR}\left(400 \mathrm{MHz}\right.$, DMSO-d $\left._{6}\right) \delta$ 0.95-1.25 (m, 5H, $\left.\mathrm{cC}_{6} \mathrm{H}_{11}\right), 1.55-1.75\left(\mathrm{~m}, 5 \mathrm{H}, \mathrm{cC}_{6} \mathrm{H}_{11}\right), 1.90(\mathrm{~m}, 1 \mathrm{H}$, $\left.\mathrm{cC}_{6} \mathrm{H}_{11}\right), 3.95\left(\mathrm{~d}, 2 \mathrm{H}, \mathrm{CH}_{2} \mathrm{cC}_{6} \mathrm{H}_{11}, J=7.2 \mathrm{~Hz}\right), 6.60$ (exch br s, $2 \mathrm{H}, \mathrm{CONH}_{2}$ ), 7.45 (m, 1H, Ar), 7.55 (exch br $\left.\mathrm{s}, 2 \mathrm{H}, \mathrm{NH}_{2}\right), 7.85(\mathrm{~m}, 1 \mathrm{H}, \mathrm{Ar}), 8.60(\mathrm{~d}, 1 \mathrm{H}, \mathrm{Ar}), 8.65(\mathrm{~s}$, $1 \mathrm{H}, \mathrm{Ar}$ ). Anal. Calcd. for $\mathrm{C}_{17} \mathrm{H}_{21} \mathrm{~N}_{5} \mathrm{O}_{2}$ : C 62.37, H 6.47, N 21.39. Found: C 62.22, H 6.48, N 21.44.

- Acetic acid 4-(5-amino-4-carbamoyl-6-oxo-3-phenyl6H-pyridazin-1-ylmethyl)-benzyl ester (7e)

A mixture of $7 \mathbf{d}(0.28 \mathrm{mmol})$ and acetyl chloride $(0.28 \mathrm{mmol})$ in anhydrous $\mathrm{CH}_{2} \mathrm{Cl}_{2}(2 \mathrm{~mL})$ was stirred 
at room temperature for $3 \mathrm{~h}$. After washing with water, the organic layer was evaporated in vacuo to afford the pure 7e as a precipitate. Yield $=90 \%(99 \mathrm{mg}) ; \mathrm{mp}=164$ $168{ }^{\circ} \mathrm{C}(\mathrm{EtOH}) .{ }^{1} \mathrm{H}-\mathrm{NMR}\left(400 \mathrm{MHz}, \mathrm{CDCl}_{3}\right) \delta 2.10(\mathrm{~s}$, $3 \mathrm{H}, \mathrm{CH}_{3}$ ), 4.95 (exch br s, $\left.1 \mathrm{H}, \mathrm{CONH}_{2}\right), 5.10$ (s, $2 \mathrm{H}$, $\mathrm{CH}_{2} \mathrm{COO}$ ), 5.30 (exch br s, $1 \mathrm{H}, \mathrm{CONH}_{2}$ ), 5.45 (s, $2 \mathrm{H}$, $\mathrm{NCH}_{2}$ ), 7.30-7.60 (m, 9H, Ar), 8.35 (exch br s, 2H, $\mathrm{NH}_{2}$ ). Anal. Calcd. for $\mathrm{C}_{21} \mathrm{H}_{20} \mathrm{~N}_{4} \mathrm{O}_{4}: \mathrm{C} 64.28, \mathrm{H} 5.14, \mathrm{~N}$ 14.28. Found: C 64.01, H 5.15, N 14.24.

General procedure for compounds 1a-j, 8 A mixture of 7a-c and 7e-k (7a and 7f are previously described) [22] (0.25$0.55 \mathrm{mmol}$ ), triethylorthoformate (or triethylorthopropionate for compound 1a) $(7.5-30 \mathrm{mmol})$ and a catalytic amount of concentrated sulfuric acid $(0.05 \mathrm{mmol})$ was heated at $130{ }^{\circ} \mathrm{C}$ for $1-5 \mathrm{~h}$ in a sealed tube. After cooling, the precipitate was recovered by filtration under vacuum. For compound $\mathbf{1 i}$ the crude precipitate was purified by flash column chromatography using $\mathrm{CH}_{2} \mathrm{Cl}_{2} / \mathrm{MeOH}$ 9:1 as eluent. For compound 1a, after dilution with cold water, the suspension was extracted with ethyl acetate $(3 \times 15 \mathrm{~mL})$. Subsequently, the solvent was evaporated in vacuo and the residue was purified by flash column chromatography using cyclohexane/ethyl acetate/ $\mathrm{MeOH}$ 1:2:0.1 as eluent.

- 7-Ethyl-5-phenyl-3,7-dihydropyrimido[4,5-d]pyridazine4,8-dione (8)

Yield $=51 \%(55 \mathrm{mg}) ; \mathrm{mp}>300{ }^{\circ} \mathrm{C}(\mathrm{EtOH}) .{ }^{1} \mathrm{H}-$ NMR (400 MHz, DMSO-d 6 ) $\delta 1.30\left(\mathrm{t}, 3 \mathrm{H}, \mathrm{CH}_{2} \mathrm{CH}_{3}\right.$, $J=7.2 \mathrm{~Hz}), 4.15\left(\mathrm{q}, 2 \mathrm{H}, \mathrm{CH}_{2} \mathrm{CH}_{3}, J=7.2 \mathrm{~Hz}\right), 7.35-7.45$ (m, 5H, Ar), 8.45 (s, 1H, Ar), 13.00 (exch br s, 1H, NH). Anal. Calcd. for $\mathrm{C}_{14} \mathrm{H}_{12} \mathrm{~N}_{4} \mathrm{O}_{2}$ : C 62.68, H 4.51, N 20.88 . Found: C 62.77, H 4.50, N 20.94.

- 2,7-Diethyl-5-phenyl-3,7-dihydropyrimido[4,5-d]pyridazine4,8-dione (1a)

Yield $=56 \%(58 \mathrm{mg}) ; \mathrm{mp}=257-260{ }^{\circ} \mathrm{C}(\mathrm{EtOH})$. ${ }^{1} \mathrm{H}-\mathrm{NMR}\left(400 \mathrm{MHz}, \mathrm{DMSO}-\mathrm{d}_{6}\right) \delta$ 1.25-1.40 (m, 6H, $\mathrm{N}-\mathrm{CH}_{2} \mathrm{CH}_{3}$ and $\mathrm{C}-\mathrm{CH}_{2} \mathrm{CH}_{3}$ ), 2.70 (q, $2 \mathrm{H}, \mathrm{C}-\mathrm{CH}_{2} \mathrm{CH}_{3}$, $J=7.2 \mathrm{~Hz}), 4.20\left(\mathrm{q}, 2 \mathrm{H}, \mathrm{N}-\mathrm{CH}_{2} \mathrm{CH}_{3}, J=7.2 \mathrm{~Hz}\right), 7.35-$ 7.50 (m, 5H, Ar), 12.90 (exch br s, 1H, NH). Anal. Calcd. for. $\mathrm{C}_{16} \mathrm{H}_{16} \mathrm{~N}_{4} \mathrm{O}_{2}$ : C 64.85, H 5.44, N 18.91. Found: C 64.68, H 5.43, N 18.96.

- 7-(4-Nitrobenzyl)-5-phenyl-3,7-dihydropyrimido[4,5-d] pyridazine-4,8-dione (1b)

Yield $=69 \%(130 \mathrm{mg}) ; \mathrm{mp}>300{ }^{\circ} \mathrm{C}$ dec. $(\mathrm{EtOH})$. ${ }^{1} \mathrm{H}-\mathrm{NMR}\left(400 \mathrm{MHz}, \mathrm{DMSO}-\mathrm{d}_{6}\right) \delta 5.50$ (s, 2H, $\mathrm{CH}_{2} \mathrm{Ar}$ ), 7.35-7.45 (m, 5H, Ar), 7.60 (d, 2H, Ar, J=8.2 Hz), 8.20 (d, 2H, Ar), 8.50 (s, 1H, Ar), 13.15 (exch br s, 1H, NH). Anal. Calcd. for. $\mathrm{C}_{19} \mathrm{H}_{13} \mathrm{~N}_{5} \mathrm{O}_{4}$ : C 60.80, H 3.49, N 18.66. Found: C 60.94, H 3.48, N 18.71.

- 4-(4,8-Dioxo-5-phenyl-4,8-dihydro-3H-pyrimido[4,5-d] pyridazin-7-ylmethyl)-benzoic acid ethyl ester (1c)
Yield $=59 \%(95 \mathrm{mg}) ; \mathrm{mp}=272-274{ }^{\circ} \mathrm{C}(\mathrm{EtOH}) .{ }^{1} \mathrm{H}-$ NMR (400 MHz, DMSO-d 6 ) $\delta 1.30\left(\mathrm{t}, 3 \mathrm{H}, \mathrm{CH}_{2} \mathrm{CH}_{3}\right.$, $J=7.2 \mathrm{~Hz}$ ), 4.30 (q, 2H, $\left.\mathrm{CH}_{2} \mathrm{CH}_{3}, J=7.2 \mathrm{~Hz}\right), 5.45$ (s, 2H, N-CH $)_{2}$, 7.35-7.45 (m, 5H, Ar), 7.50 (d, 2H, Ar, $J=8.4 \mathrm{~Hz}$ ), 7.95 (d, 2H, Ar, $J=8.1 \mathrm{~Hz}), 8.45$ (s, 1H, Ar), 13.15 (exch br s, $1 \mathrm{H}, \mathrm{NH}$ ). Anal. Calcd. for. $\mathrm{C}_{22} \mathrm{H}_{18} \mathrm{~N}_{4} \mathrm{O}_{4}$ : C 65.66, H 4.51, N 13.92. Found: C 65.45, H 4.52, N 13.96.

- Acetic acid 4-(4,8-dioxo-5-phenyl-4,8-dihydro3H-pyrimido[4,5-d]pyridazin-7-ylmethyl)benzyl ester (1d)

Yield $=44 \%(53 \mathrm{mg}) ; \mathrm{mp}=240-243{ }^{\circ} \mathrm{C}(\mathrm{EtOH}) .{ }^{1} \mathrm{H}-$ NMR (400 MHz, DMSO-d 6 ) $\delta 2.05$ (s, 3H, $\mathrm{CH}_{3}$ ), 5.05 (s, 2H, $\left.\mathrm{CH}_{2} \mathrm{OCO}\right), 5.35$ (s, 2H, N-CH $), 7.30-7.45$ (m, 9H, Ar), 8.45 (s, 1H, Ar), 13.10 (exch br s, 1H, NH). Anal. Calcd. for. $\mathrm{C}_{22} \mathrm{H}_{18} \mathrm{~N}_{4} \mathrm{O}_{4}$ : C 65.66, H 4.51, N 13.92 . Found: C 65.49, H 4.51, N 13.95.

- 7-Ethyl-5-(3-fluorophenyl)-3,7-dihydro-pyrimido[4,5-d] pyridazine-4,8-dione (1e)

Yield $=72 \%(93 \mathrm{mg}) ; \mathrm{mp}>300{ }^{\circ} \mathrm{C}(\mathrm{EtOH}) .{ }^{1} \mathrm{H}-$ NMR (400 MHz, DMSO-d 6 ) $\delta 1.30\left(\mathrm{t}, 3 \mathrm{H}, \mathrm{CH}_{2} \mathrm{CH}_{3}\right.$, $J=7.2 \mathrm{~Hz}), 4.20\left(\mathrm{q}, 2 \mathrm{H}, \mathrm{CH}_{2} \mathrm{CH}_{3}, J=7.2 \mathrm{~Hz}\right), 7.20$ $7.30(\mathrm{~m}, 3 \mathrm{H}, \mathrm{Ar}), 7.40-7.50(\mathrm{~m}, 1 \mathrm{H}, \mathrm{Ar}), 8.45(\mathrm{~s}$, $1 \mathrm{H}, \mathrm{Ar}$ ), 13.10 (exch br s, 1H, NH). Anal. Calcd. for. $\mathrm{C}_{14} \mathrm{H}_{11} \mathrm{FN}_{4} \mathrm{O}_{2}$ : C 58.74, H 3.87, N 19.57. Found: C 58.88, H 3.88, N 19.51 .

- 5-(3-Chlorophenyl)-7-cyclopropylmethyl-3,7dihydropyrimido[4,5-d]pyridazine-4,8-dione (1f)

Yield $=38 \%(69 \mathrm{mg}) ; \mathrm{mp}>300{ }^{\circ} \mathrm{C}(\mathrm{EtOH}) .{ }^{1} \mathrm{H}-\mathrm{NMR}$ $\left(400 \mathrm{MHz}, \mathrm{DMSO}-\mathrm{d}_{6}\right) \delta 0.35-0.55\left(\mathrm{~m}, 4 \mathrm{H}, \mathrm{cC}_{3} \mathrm{H}_{5}\right), 1.30$ $\left(\mathrm{m}, 1 \mathrm{H}, \mathrm{cC}_{3} \mathrm{H}_{5}\right), 4.00\left(\mathrm{~d}, 2 \mathrm{H}, \mathrm{CH}_{2} \mathrm{c} \mathrm{cC}_{3} \mathrm{H}_{5}, J=6.8 \mathrm{~Hz}\right)$, 7.35-7.50 (m, 4H, Ar), 8.45 (s, 1H, Ar), 13.15 (exch br s, $1 \mathrm{H}, \mathrm{NH})$. Anal. Calcd. for. $\mathrm{C}_{16} \mathrm{H}_{13} \mathrm{ClN}_{4} \mathrm{O}_{2}$ : C 58.46, $\mathrm{H}$ 3.99, N 17.04. Found: C 58.65, H 3.98, N 17.08.

- 5-(3-Chlorophenyl)-7-cyclohexylmethyl-3,7dihydropyrimido[4,5-d]pyridazine-4,8-dione (1 g)

Yield $=20 \%(40 \mathrm{mg}) ; \mathrm{mp}=271-274{ }^{\circ} \mathrm{C}(\mathrm{EtOH})$. ${ }^{1} \mathrm{H}-\mathrm{NMR}\left(400 \mathrm{MHz}, \mathrm{DMSO}-\mathrm{d}_{6}\right) \delta$ 0.95-1.25 (m, 5H, $\left.\mathrm{cC}_{6} \mathrm{H}_{11}\right), 1.55-1.70\left(\mathrm{~m}, 5 \mathrm{H}, \mathrm{cC}_{6} \mathrm{H}_{11}\right), 1.90(\mathrm{~m}, 1 \mathrm{H}$, $\left.\mathrm{cC}_{6} \mathrm{H}_{11}\right), 4.00\left(\mathrm{~d}, 2 \mathrm{H}, \mathrm{CH}_{2} \mathrm{cC}_{6} \mathrm{H}_{11}, J=7.2 \mathrm{~Hz}\right), 7.35-$ 7.50 (m, 4H, Ar), 8.45 (s, 1H, Ar), 13.10 (exch br s, 1H, $\mathrm{NH})$. Anal. Calcd. for. $\mathrm{C}_{19} \mathrm{H}_{19} \mathrm{ClN}_{4} \mathrm{O}_{2}$ : C 61.54, H 5.16, N 15.11 Found: C 61.37, H 5.18, N 15.16.

- 5-(3-Chlorophenyl)-7-cyclohexyl-3,7-dihydropyrimido[4,5-d] pyridazine-4,8-dione $(\mathbf{1 ~ h})$

Yield $=58 \%(73 \mathrm{mg}) ; \mathrm{mp}=289-291{ }^{\circ} \mathrm{C}\left(\mathrm{Et}_{2} \mathrm{O}\right) .{ }^{1} \mathrm{H}-$ NMR (400 MHz, DMSO-d $\left.\mathrm{d}_{6}\right) \delta 1.20\left(\mathrm{~m}, 1 \mathrm{H}, \mathrm{cC}_{6} \mathrm{H}_{11}\right)$, $1.40\left(\mathrm{~m}, 2 \mathrm{H}, \mathrm{cC}_{6} \mathrm{H}_{11}\right), 1.60-1.90\left(\mathrm{~m}, 7 \mathrm{H}, \mathrm{cC}_{6} \mathrm{H}_{11}\right), 4.85$ (t, $1 \mathrm{H}, \mathrm{cC}_{6} \mathrm{H}_{11}, J=7.0 \mathrm{~Hz}$ ), 7.35-7.50 (m, 4H, Ar), 8.45 (s, 1H, Ar), 13.10 (exch br s, 1H, NH). Anal. Calcd. for. $\mathrm{C}_{18} \mathrm{H}_{17} \mathrm{ClN}_{4} \mathrm{O}_{2}: \mathrm{C} 60.59, \mathrm{H} 4.80, \mathrm{~N}$ 15.70. Found: $\mathrm{C}$ 60.81, H 4.79, N 15.32. 
- 7-Cyclohexylmethyl-5-m-tolyl-3,7-dihydropyrimido[4,5d]pyridazine-4,8-dione (1i)

Yield $=20 \%(40 \mathrm{mg}) ; \mathrm{mp}=219-222{ }^{\circ} \mathrm{C}\left(\mathrm{Et}_{2} \mathrm{O}\right)$. ${ }^{1} \mathrm{H}-\mathrm{NMR}\left(400 \mathrm{MHz}\right.$, DMSO-d $\left.{ }_{6}\right) \delta$ 0.95-1.30 (m, 5H, $\left.\mathrm{cC}_{6} \mathrm{H}_{11}\right), 1.60-1.75\left(\mathrm{~m}, 5 \mathrm{H}, \mathrm{cC}_{6} \mathrm{H}_{11}\right), 1.90(\mathrm{~m}, 1 \mathrm{H}$, $\left.\mathrm{cC}_{6} \mathrm{H}_{11}\right), 2.35\left(\mathrm{~s}, 3 \mathrm{H}, \mathrm{CH}_{3}\right), 4.00\left(\mathrm{~d}, 2 \mathrm{H}, \mathrm{CH}_{2} \mathrm{cC}_{6} \mathrm{H}_{11}\right.$, $J=7.2 \mathrm{~Hz}$ ), 7.15-7.30 (m, 4H, Ar), 8.40 (s, 1H, Ar), 13.05 (exch br s, $1 \mathrm{H}, \mathrm{NH}$ ). Anal. Calcd. for. $\mathrm{C}_{20} \mathrm{H}_{22} \mathrm{~N}_{4} \mathrm{O}_{2}$ : C 68.55, H 6.33, N 15.99 Found: C 68.41, H 6.35, N 15.95 .

- 7-Cyclohexylmethyl-5-pyridin-3-yl-3,7dihydropyrimido[4,5-d]pyridazine-4,8-dione (1j)

Yield $=20 \%(30 \mathrm{mg}) ; \mathrm{mp}>300{ }^{\circ} \mathrm{C}\left(\mathrm{Et}_{2} \mathrm{O}\right) .{ }^{1} \mathrm{H}-\mathrm{NMR}$ (400 MHz, DMSO-d $\left.{ }_{6}\right) \delta 0.95-1.30\left(\mathrm{~m}, 5 \mathrm{H}, \mathrm{cC}_{6} \mathrm{H}_{11}\right)$, 1.55-1.75 (m, 5H, $\left.\mathrm{cC}_{6} \mathrm{H}_{11}\right), 1.90\left(\mathrm{~m}, 1 \mathrm{H}, \mathrm{cC}_{6} \mathrm{H}_{11}\right), 4.00$ $\left(\mathrm{d}, 2 \mathrm{H}, \mathrm{CH}_{2} \mathrm{cC}_{6} \mathrm{H}_{11}, J=7.2 \mathrm{~Hz}\right), 7.45(\mathrm{~m}, 1 \mathrm{H}, \mathrm{Ar})$, $7.80(\mathrm{~d}, 1 \mathrm{H}, \mathrm{Ar}, J=8.2 \mathrm{~Hz}), 8.45$ (s, $1 \mathrm{H}, \mathrm{Ar}), 8.60$ (m, $2 \mathrm{H}, \mathrm{Ar}$ ), 13.05 (exch br s, 1H, NH). Anal. Calcd. for. $\mathrm{C}_{18} \mathrm{H}_{19} \mathrm{~N}_{5} \mathrm{O}_{2}$ : C 64.08, H 5.68, N 20.76. Found: C 64.27, H 5.66, N 20.69 .

General procedure for compounds 1 k, I Compounds $1 \mathbf{k , l}$ were obtained from 8 by adopting the general procedure described for intermediate of type 7. After dilution with cold water, compound $\mathbf{1} \mathbf{k}$ was recovered by filtration under vacuum. For compound $\mathbf{1} \mathbf{l}$, the suspension was extracted with $\mathrm{CH}_{2} \mathrm{Cl}_{2}(3 \times 15 \mathrm{~mL})$ and the solvent was evaporated in vacuo.

- 7-Ethyl-3-methyl-5-phenyl-3,7-dihydropyrimido[4,5-d] pyridazine-4,8-dione $(\mathbf{1 ~ k})$

Yield $=26 \%(37 \mathrm{mg}) ; \mathrm{mp}=237-239{ }^{\circ} \mathrm{C}(\mathrm{EtOH})$. ${ }^{1} \mathrm{H}-\mathrm{NMR}\left(400 \mathrm{MHz}, \mathrm{CDCl}_{3}\right) \delta 1.45\left(\mathrm{t}, 3 \mathrm{H}, \mathrm{CH}_{2} \mathrm{CH}_{3}\right.$, $J=7.2 \mathrm{~Hz}), 3.55\left(\mathrm{~s}, 3 \mathrm{H}, \mathrm{N}-\mathrm{CH}_{3}\right), 4.40(\mathrm{q}, 2 \mathrm{H}$, $\left.\mathrm{N}-\mathrm{CH}_{2} \mathrm{CH}_{3}, J=7.2 \mathrm{~Hz}\right), 7.40-7.50(\mathrm{~m}, 5 \mathrm{H}, \mathrm{Ar}), 8.40(\mathrm{~s}$, $1 \mathrm{H}, \mathrm{Ar}$ ). Anal. Calcd. for. $\mathrm{C}_{15} \mathrm{H}_{14} \mathrm{~N}_{4} \mathrm{O}_{2}: \mathrm{C} 63.82$, H 5.00, N 19.85. Found: C 63.96, H 5.01, N 19.91.

- 3-Benzyl-7-ethyl-5-phenyl-3,7-dihydropyrimido[4,5-d] pyridazine-4,8-dione (1 l l)

Yield $=46 \%(83 \mathrm{mg}) ; \mathrm{mp}=217-219{ }^{\circ} \mathrm{C}(\mathrm{EtOH})$. ${ }^{1} \mathrm{H}-\mathrm{NMR}\left(400 \mathrm{MHz}, \mathrm{CDCl}_{3}\right) \delta 1.45\left(\mathrm{t}, 3 \mathrm{H}, \mathrm{CH}_{2} \mathrm{CH}_{3}\right.$, $J=7.2 \mathrm{~Hz}), 4.40\left(\mathrm{q}, 2 \mathrm{H}, \mathrm{CH}_{2} \mathrm{CH}_{3}, J=7.2 \mathrm{~Hz}\right), 5.15(\mathrm{~s}$, $\left.2 \mathrm{H}, \mathrm{CH}_{2} \mathrm{Ph}\right), 7.30-7.50$ (m, 10H, Ar), 8.45 (s, 1H, Ar). Anal. Calcd. for. $\mathrm{C}_{21} \mathrm{H}_{18} \mathrm{~N}_{4} \mathrm{O}_{2}$ : C 70.38, H 5.06, N 15.63. Found: C 70.54, H 5.05, N 15.68.

General procedure for compounds $1 \mathrm{~m}, \mathrm{n}$ A mixture of $1 \mathrm{c}$ or 1d $(0.12 \mathrm{mmol})$, ethanol $(1.5 \mathrm{~mL})$ and $6 \mathrm{~N} \mathrm{NaOH}(0.5 \mathrm{~mL})$ was stirred at room temperature for 1-2 h. After evaporation of the solvent and dilution with cold water the suspension was acidified with $6 \mathrm{~N} \mathrm{HCl}$. For compound $1 \mathbf{~ m}$, the mixture was extracted with ethyl acetate $(3 \times 15 \mathrm{~mL})$ and evaporation of the solvent afforded desiderated compound.
For compound 1n, the solid was recovered by filtration under vacuum.

- 4-(4,8-Dioxo-5-phenyl-4,8-dihydro-3H-pyrimido[4,5-d] pyridazin-7-ylmethyl)-benzoic acid $(\mathbf{1} \mathrm{m})$

Yield $=25 \%(15 \mathrm{mg}) ; \mathrm{mp}=295-298{ }^{\circ} \mathrm{C}$ dec. $(\mathrm{EtOH})$. ${ }^{1} \mathrm{H}-\mathrm{NMR}\left(400 \mathrm{MHz}, \mathrm{DMSO}-\mathrm{d}_{6}\right) \delta 5.45\left(\mathrm{~s}, 2 \mathrm{H}, \mathrm{CH}_{2}\right)$, 7.35-7.50 (m, 7H, Ar), 7.90 (d, 2H, Ar, $J=8.4 \mathrm{~Hz}$ ), 8.45 (s, 1H, Ar), 13.15 (exch br s, 1H, NH). Anal. Calcd. for. $\mathrm{C}_{20} \mathrm{H}_{14} \mathrm{~N}_{4} \mathrm{O}_{4}$ : C 64.17, H 3.77, N 14.97. Found: C 64.36, $\mathrm{H} 3.78, \mathrm{~N} 15.02$.

- 7-(4-Hydroxymethylbenzyl)-5-phenyl-3,7dihydropyrimido[4,5-d]pyridazine-4,8-dione (In)

Yield $=65 \%(30 \mathrm{mg}) ; \mathrm{mp}=170-174{ }^{\circ} \mathrm{C}$ dec. $(\mathrm{EtOH})$. ${ }^{1} \mathrm{H}-\mathrm{NMR}\left(400 \mathrm{MHz}, \mathrm{DMSO}-\mathrm{d}_{6}\right) \delta 4.45\left(\mathrm{~d}, 2 \mathrm{H}, \mathrm{CH}_{2} \mathrm{OH}\right.$, $J=5.6 \mathrm{~Hz}$ ), 5.15 (exch br t, $1 \mathrm{H}, \mathrm{CH}_{2} \mathrm{OH}, J=5.6 \mathrm{~Hz}$ ), 5.35 (s, 2H, $\mathrm{CH}_{2}$ ), 7.25-7.45 (m, 9H, Ar), 8.45 (s, 1H, Ar), 13.10 (exch br s, $1 \mathrm{H}, \mathrm{NH}$ ). Anal. Calcd. for $\mathrm{C}_{20} \mathrm{H}_{16} \mathrm{~N}_{4} \mathrm{O}_{3}$ : C 66.66, H 4.48, N 15.55. Found: C 66.82, H 4.49, N 15.49 .

- 5-Amino-6-oxo-3-pyridin-3-yl-1,6-dihydropyridazine4-carboxylic acid methyl ester $(\mathbf{9 b})$

A mixture of isoxazolopyridazinone $5 \mathrm{e}(0.46 \mathrm{mmol})$ and piperidine $(0.23 \mathrm{mmol})$ in anhydrous methanol $(6 \mathrm{~mL})$ was stirred at $65^{\circ} \mathrm{C}$ for $1.5 \mathrm{~h}$. After evaporation of the solvent, the mixture was diluted with cold water (5 mL), neutralised with $2 \mathrm{~N} \mathrm{HCl}$ and extracted with $\mathrm{CH}_{2} \mathrm{Cl}_{2}(3 \times 15 \mathrm{~mL})$. Evaporation of the solvent afforded the final compound $9 \mathrm{~b}$. Yield $=46 \%(52 \mathrm{mg}) ; \mathrm{mp}=210$ $212{ }^{\circ} \mathrm{C}(\mathrm{EtOH}) .{ }^{1} \mathrm{H}-\mathrm{NMR}\left(400 \mathrm{MHz}, \mathrm{CDCl}_{3}\right) \delta 3.55$ (s, $3 \mathrm{H}, \mathrm{CH}_{3} \mathrm{O}$ ), 6.50 (exch br s, $2 \mathrm{H}, \mathrm{NH}_{2}$ ), 7.25-7.45 (m, $1 \mathrm{H}$, Ar), 7.65 (m, 1H, Ar), 8.60 (m, 2H, Ar), 11.90 (exch br s, $1 \mathrm{H}, \mathrm{NH}$ ). Anal. Calcd. for $\mathrm{C}_{11} \mathrm{H}_{10} \mathrm{~N}_{4} \mathrm{O}_{3}$ : C 53.66, H 4.09, N 22.75. Found: C 53.83, H 4.08, N 22.71.

General procedure for compounds 10a-d Compounds 10ad were obtained from 9a,b adopting the general procedure described intermediate of type 7. After dilution with water, the suspension was extracted with $\mathrm{CH}_{2} \mathrm{Cl}_{2}(3 \times 15 \mathrm{~mL})$. The organic layer was recovered, dried on sodium sulfate and evaporated to afford the pure compounds $10 \mathrm{a}$ and $10 \mathrm{c}$, while compounds 10b,d were purified by flash column chromatography using cyclohexane/ethyl acetate 1:2 as eluent.

- 5-Amino-1-cyclohexylmethyl-6-oxo-3-phenyl-1,6dihydropyridazine-4-carboxylic acid methyl ester (10a) Yield $=50 \%(85 \mathrm{mg}) ; \mathrm{mp}=134-136{ }^{\circ} \mathrm{C}(\mathrm{EtOH}) .{ }^{1} \mathrm{H}-$ NMR $\left(400 \mathrm{MHz}, \mathrm{CDCl}_{3}\right) \delta 1.05-1.30\left(\mathrm{~m}, 5 \mathrm{H}, \mathrm{cC}_{6} \mathrm{H}_{11}\right)$, 1.65-1.75 (m, 5H, $\left.\mathrm{cC}_{6} \mathrm{H}_{11}\right), 2.00\left(\mathrm{~m}, 1 \mathrm{H}, \mathrm{cC}_{6} \mathrm{H}_{11}\right), 3.50$ (s, $\left.3 \mathrm{H}, \mathrm{CH}_{3} \mathrm{O}\right), 4.05\left(\mathrm{~d}, 2 \mathrm{H}, \mathrm{CH}_{2} \mathrm{cC}_{6} \mathrm{H}_{11}, J=7.0 \mathrm{~Hz}\right)$, 7.05 (exch br s, $2 \mathrm{H}, \mathrm{NH}_{2}$ ), 7.35-7.45 (m, 5H, Ar). Anal. Calcd. for $\mathrm{C}_{19} \mathrm{H}_{23} \mathrm{~N}_{3} \mathrm{O}_{3}: \mathrm{C} 66.84, \mathrm{H} 6.79, \mathrm{~N} 12.31$. Found: C 66.63, H 6.81, N 12.34 . 
- 5-Amino-1-benzyl-6-oxo-3-phenyl-1,6-dihydro-pyridazine4-carboxylic acid methyl ester (10b)

Yield $=77 \%(130 \mathrm{mg}) ; \mathrm{mp}=145-147{ }^{\circ} \mathrm{C}(\mathrm{EtOH}) .{ }^{1} \mathrm{H}-$ NMR (400 MHz, $\left.\mathrm{CDCl}_{3}\right) \delta 3.50\left(\mathrm{~s}, 3 \mathrm{H}, \mathrm{CH}_{3} \mathrm{O}\right), 5.35$ (s, $2 \mathrm{H}, \mathrm{CH}_{2} \mathrm{Ph}$ ), 7.05 (exch br s, $2 \mathrm{H}, \mathrm{NH}_{2}$ ), 7.30-7.50 (m, $10 \mathrm{H}, \mathrm{Ar}$ ). Anal. Calcd. for $\mathrm{C}_{19} \mathrm{H}_{17} \mathrm{~N}_{3} \mathrm{O}_{3}$ : C 68.05, H 5.11, N 12.53. Found: C 68.28, H 5.10, N 12.49.

- 5-Amino-1-cyclopropylmethyl-6-oxo-3-phenyl-1,6-dihydropyridazine-4-carboxylic acid methyl ester $(\mathbf{1 0 c})$

Yield $=40 \%(115 \mathrm{mg}) ; \mathrm{mp}=119-121^{\circ} \mathrm{C}(\mathrm{EtOH}) .{ }^{1} \mathrm{H}-$ NMR $\left(400 \mathrm{MHz}, \mathrm{CDCl}_{3}\right) \delta 0.45-0.60\left(\mathrm{~m}, 4 \mathrm{H}, \mathrm{cC}_{3} \mathrm{H}_{5}\right)$, $1.35-1.50\left(\mathrm{~m}, 1 \mathrm{H}, \mathrm{cC}_{3} \mathrm{H}_{5}\right), 3.50\left(\mathrm{~s}, 3 \mathrm{H}, \mathrm{CH}_{3} \mathrm{O}\right), 4.05(\mathrm{~d}$, $2 \mathrm{H}, \mathrm{CH}_{2} \mathrm{CC}_{3} \mathrm{H}_{5}, J=6.8 \mathrm{~Hz}$ ), 7.05 (exch br s, $2 \mathrm{H}, \mathrm{NH}_{2}$ ), 7.40 (s, 5H, Ar). Anal. Calcd. for $\mathrm{C}_{16} \mathrm{H}_{17} \mathrm{~N}_{3} \mathrm{O}_{3}: \mathrm{C} 64.20$, H 5.72, N 14.04. Found: C 64.37, H 5.70, N 14.08.

- 5-Amino-1-(3-chlorobenzyl)-6-oxo-3-pyridin-3-yl1,6-dihydropyridazine-4-carboxylic acid methyl ester (10d)

Yield $=75 \%(140 \mathrm{mg}) ; \mathrm{mp}=197-199^{\circ} \mathrm{C}(\mathrm{EtOH}) .{ }^{1} \mathrm{H}-$ NMR (400 MHz, $\left.\mathrm{CDCl}_{3}\right) \delta 3.65\left(\mathrm{~s}, 3 \mathrm{H}, \mathrm{CH}_{3} \mathrm{O}\right), 5.30$ (s, $2 \mathrm{H}, \mathrm{NCH}_{2}$ ), 7.00 (exch br s, $2 \mathrm{H}, \mathrm{CONH}_{2}$ ), 7.30 (s, $3 \mathrm{H}$, Ar), 7.45 (s, 1H, Ar), 7.95 (m, 1H, Ar), 8.30 (m, 1H, Ar), $8.80(\mathrm{~m}, 2 \mathrm{H}, \mathrm{Ar})$. Anal. Calcd. for. $\mathrm{C}_{18} \mathrm{H}_{15} \mathrm{ClN}_{4} \mathrm{O}_{3}$ : C 58.31, H 4.08, N 15.11. Found: C 58.15, H 4.09, N 15.16.

General procedure for compounds 11a-d Compounds 11ad were obtained starting from 10a-d $(1.2 \mathrm{mmol})$, following the same procedure described for $\mathbf{1} \mathbf{m}, \mathbf{n}$. In this case, the mixture was heated at $60-70{ }^{\circ} \mathrm{C}$ for $1-2 \mathrm{~h}$. After acidification with $6 \mathrm{~N} \mathrm{HCl}$, the final compounds were recovered by filtration under vacuum.

- 5-Amino-1-cyclohexylmethyl-6-oxo-3-phenyl-1,6-dihydropyridazine-4-carboxylic acid (11a)

Yield $=93 \%(370 \mathrm{mg}) ; \mathrm{mp}=224-226{ }^{\circ} \mathrm{C}$ dec. (Cyclohexane). ${ }^{1} \mathrm{H}-\mathrm{NMR}\left(400 \mathrm{MHz}, \mathrm{CDCl}_{3}\right) \delta 1.05-1.30$ $\left(\mathrm{m}, 5 \mathrm{H}, \mathrm{cC}_{6} \mathrm{H}_{11}\right), 1.65-1.75\left(\mathrm{~m}, 5 \mathrm{H}, \mathrm{cC}_{6} \mathrm{H}_{11}\right), 2.00(\mathrm{~m}$, $\left.1 \mathrm{H}, \mathrm{cC}_{6} \mathrm{H}_{11}\right), 4.05\left(\mathrm{~d}, 2 \mathrm{H}, \mathrm{N}-\mathrm{CH}_{2}, J=7.3 \mathrm{~Hz}\right.$ ), 5.05 (exch br s, $1 \mathrm{H}, \mathrm{OH}$ ), 7.45 (s, 5H, Ar), 8.05 (exch br s, 2H, $\mathrm{NH}_{2}$ ). Anal. Calcd. for $\mathrm{C}_{18} \mathrm{H}_{21} \mathrm{~N}_{3} \mathrm{O}_{3}: \mathrm{C} 66.04, \mathrm{H} 6.47, \mathrm{~N}$ 12.84. Found: C 66.25, H 6.45, N 12.88.

- 5-Amino-1-benzyl-6-oxo-3-phenyl-1,6-dihydro-pyridazine4-carboxylic acid (11b)

Yield $=42 \%(160 \mathrm{mg}) ; \mathrm{mp}=207-211^{\circ} \mathrm{C}$ dec. $(\mathrm{EtOH})$. ${ }^{1} \mathrm{H}-\mathrm{NMR}\left(400 \mathrm{MHz}, \mathrm{CDCl}_{3}\right) \delta 5.35\left(\mathrm{~s}, 2 \mathrm{H}, \mathrm{NCH}_{2}\right), 5.15$ (exch br s, 1H, OH), 7.30-7.50 (m, 10H, Ar), 7.80 (exch br s, $2 \mathrm{H}, \mathrm{NH}_{2}$ ). Anal. Calcd. for $\mathrm{C}_{18} \mathrm{H}_{15} \mathrm{~N}_{3} \mathrm{O}_{3}$ : C 67.28, H 4.71, N 13.08. Found: C 67.46, H 4.70, N 13.04 .

- 5-Amino-1-cyclopropylmethyl-6-oxo-3-phenyl-1,6-dihydropyridazine-4-carboxylic acid (11c)

Yield $=90 \%$ (310 mg); $\mathrm{mp}=203-206{ }^{\circ} \mathrm{C} \mathrm{dec}$. $(\mathrm{EtOH})$. ${ }^{1} \mathrm{H}-\mathrm{NMR}\left(400 \mathrm{MHz}, \mathrm{CDCl}_{3}\right) \delta$ 0.45-0.60 (m, 4H, $\left.\mathrm{cC}_{3} \mathrm{H}_{5}\right) 1.35-1.45\left(\mathrm{~m}, 1 \mathrm{H}, \mathrm{cC}_{3} \mathrm{H}_{5}\right), 4.05\left(\mathrm{~d}, 2 \mathrm{H}, \mathrm{N}-\mathrm{CH}_{2}\right.$,
$J=7.2 \mathrm{~Hz}$ ), 5.70 (exch br s, 1H, OH), 7.45 (s, 5H, Ar), 7.75 (exch br s, $2 \mathrm{H}, \mathrm{NH}_{2}$ ). Anal. Calcd. for $\mathrm{C}_{15} \mathrm{H}_{15} \mathrm{~N}_{3} \mathrm{O}_{3}$ : C 63.15, H 5.30, N 14.73. Found: C 63.38, H 5.28, N 14.70 .

- 5-Amino-1-(3-chlorobenzyl)-6-oxo-3-pyridin-3-yl1,6-dihydropyridazine-4-carboxylic acid (11d)

Yield $=98 \%(440 \mathrm{mg}) ; \mathrm{mp}=228-232{ }^{\circ} \mathrm{C} \mathrm{dec} .\left(\mathrm{Et}_{2} \mathrm{O}\right) .{ }^{1} \mathrm{H}-$ NMR (400 MHz, DMSO-d $\left.{ }_{6}\right) \delta 5.30\left(\mathrm{~s}, 2 \mathrm{H}, \mathrm{N}_{-} \mathrm{CH}_{2}\right), 7.30(\mathrm{~d}$, $1 \mathrm{H}, \mathrm{Ar}, J=8.2 \mathrm{~Hz}$ ), 6.95 (exch br s, 1H, OH), 7.35-7.45 (m, $4 \mathrm{H}, \mathrm{Ar}), 7.75$ (d, 1H, Ar, J=8.4 Hz), 8.55 (m, 2H, Ar), 8.70 (exch br s, $2 \mathrm{H}, \mathrm{CONH}_{2}$ ). Anal. Calcd. for $\mathrm{C}_{18} \mathrm{H}_{15} \mathrm{ClN}_{4} \mathrm{O}_{3}: \mathrm{C}$ 58.31, H 4.08, N 15.11. Found: C 58.48, H 4.07, N 15.16.

General procedure for compounds 12a-d A catalytic amount of $\mathrm{Et}_{3} \mathrm{~N}$ was added to a cold suspension of 11a-d $(0.4 \mathrm{mmol})$ in $\mathrm{SOCl}_{2}(1.5-3 \mathrm{~mL})$ and the mixture was refluxed for $1 \mathrm{~h}$. The excess of $\mathrm{SOCl}_{2}$ was removed in vacuo. After cooling, $33 \% \mathrm{NH}_{4} \mathrm{OH}(4-6 \mathrm{~mL})$ was added and the suspension was stirred for $0.5-1 \mathrm{~h}$. Ice-water $(10-15 \mathrm{~mL})$ was added to the residue and the final compounds $\mathbf{1 2 b}$ and $\mathbf{1 2 c}$ were recovered by filtration under vacuum. For compounds 12a and 12d, the mixture was extracted with ethyl acetate $(3 \times 15 \mathrm{~mL})$ and the solvent was evaporated in vacuo to afford pure final compounds, while 12a was purified by flash column chromatography using cyclohexane/ethyl acetate 1:3 as eluent.

- 5-Amino-1-cyclohexylmethyl-6-oxo-3-phenyl-1,6-dihydropyridazine-4-carboxylic acid amide (12a)

Yield $=66 \%(86 \mathrm{mg}) ; \mathrm{mp}=179-181^{\circ} \mathrm{C}($ Cyclohexane). ${ }^{1} \mathrm{H}-\mathrm{NMR}\left(400 \mathrm{MHz}, \mathrm{CDCl}_{3}\right) \delta 1.05-1.30(\mathrm{~m}, 5 \mathrm{H}$, $\left.\mathrm{cC}_{6} \mathrm{H}_{11}\right), 1.65-1.80\left(\mathrm{~m}, 5 \mathrm{H}, \mathrm{cC}_{6} \mathrm{H}_{11}\right), 2.00-2.10(\mathrm{~m}, 1 \mathrm{H}$, $\left.\mathrm{cC}_{6} \mathrm{H}_{11}\right), 4.05\left(\mathrm{~d}, 2 \mathrm{H}, \mathrm{CH}_{2} \mathrm{cC}_{6} \mathrm{H}_{11}, J=7.6 \mathrm{~Hz}\right), 4.95$ (exch br s, $1 \mathrm{H}, \mathrm{CONH}_{2}$ ), 5.45 (exch br s, $1 \mathrm{H}, \mathrm{CONH}_{2}$ ), 7.45-7.55 (m, 5H, Ar), 8.15 (exch br s, $2 \mathrm{H}, \mathrm{NH}_{2}$ ). Anal. Calcd. for $\mathrm{C}_{18} \mathrm{H}_{22} \mathrm{~N}_{4} \mathrm{O}_{2}$ : C 66.24, H 6.79, $\mathrm{N}$ 17.17. Found: C 66.45, H 6.77, N 17.11.

- 5-Amino-1-benzyl-6-oxo-3-phenyl-1,6-dihydro-pyridazine4-carboxylic acid amide (12b)

Yield $=30 \%(40 \mathrm{mg}) ; \mathrm{mp}=218-221{ }^{\circ} \mathrm{C} \mathrm{dec}$. $(\mathrm{EtOH})$. ${ }^{1} \mathrm{H}-\mathrm{NMR}\left(400 \mathrm{MHz}, \mathrm{CDCl}_{3}\right.$ ) $\delta 4.95$ (exch br s, $1 \mathrm{H}$, $\left.\mathrm{CONH}_{2}\right), 5.30$ (exch br s, $\left.1 \mathrm{H}, \mathrm{CONH}_{2}\right), 5.35(\mathrm{~s}, 2 \mathrm{H}$, $\mathrm{CH}_{2} \mathrm{Ph}$ ), 7.30-7.55 (m, 10H, Ar), 8.25 (exch br s, $2 \mathrm{H}$, $\mathrm{NH}_{2}$ ). Anal. Calcd. for $\mathrm{C}_{18} \mathrm{H}_{16} \mathrm{~N}_{4} \mathrm{O}_{2}$ : C 67.49, H 5.03, N 17.49. Found: C 67.26, H 5.04, N 17.46.

- 5-Amino-1-cyclopropylmethyl-6-oxo-3-phenyl-1,6-dihydropyridazine-4-carboxylic acid amide (12c)

Yield $=55 \%(63 \mathrm{mg}) ; \mathrm{mp}=203-206{ }^{\circ} \mathrm{C}(\mathrm{EtOH}) .{ }^{1} \mathrm{H}-$ NMR (400 MHz, $\left.\mathrm{CDCl}_{3}\right) \delta 0.45-0.60\left(\mathrm{~m}, 4 \mathrm{H}, \mathrm{cC}_{3} \mathrm{H}_{5}\right)$, 1.35-1.50 (m, $\left.1 \mathrm{H}, \mathrm{cC}_{3} \mathrm{H}_{5}\right), 4.05\left(\mathrm{~d}, 2 \mathrm{H}, \mathrm{CH}_{2} \mathrm{cC}_{3} \mathrm{H}_{5}\right.$, $J=7.2 \mathrm{~Hz}$ ), 5.00 (exch br s, $1 \mathrm{H}, \mathrm{CONH}_{2}$ ), 5.40 (exch br s, $1 \mathrm{H}, \mathrm{CONH}_{2}$ ), 7.45-7.60 (m, 5H, Ar), 7.90 (exch br s, 2H, NH2). Anal. Calcd. for $\mathrm{C}_{18} \mathrm{H}_{16} \mathrm{~N}_{4} \mathrm{O}_{2}: \mathrm{C} 67.49, \mathrm{H}$ 5.03, N 17.49. Found: C 67.26, H 5.04, N 17.46. 
- 5-Amino-1-(3-chlorobenzyl)-6-oxo-3-pyridin-3-yl-1,6dihydro-pyridazine-4-carboxylic acid amide (12d)

Yield $=72 \%(102 \mathrm{mg}) ; \mathrm{mp}=235-238{ }^{\circ} \mathrm{C}$ dec. (EtOH). ${ }^{1} \mathrm{H}-\mathrm{NMR}\left(400 \mathrm{MHz}, \mathrm{DMSO}-\mathrm{d}_{6}\right) \delta 5.30$ (s, 2H, $\mathrm{NCH}_{2} \mathrm{Ar}$ ), 6.70 (exch br s, 2H, $\mathrm{NH}_{2}$ ), 7.30-7.45 (m, 5H, Ar), 7.50-7.60 (exch br s, 2H, $\mathrm{CONH}_{2}$ ), 7.85 (d, $1 \mathrm{H}, \mathrm{Ar}, J=8.2 \mathrm{~Hz}$ ), 7.90 (exch br s, $2 \mathrm{H}, \mathrm{NH}_{2}$ ), 8.60 (d, $1 \mathrm{H}, \mathrm{Ar}, J=8.4 \mathrm{~Hz}$ ), 8.65 (s, 1H, Ar). Anal. Calcd. for $\mathrm{C}_{17} \mathrm{H}_{14} \mathrm{ClN}_{5} \mathrm{O}_{2}$ : C 57.39, H 3.97, N, 19.68. Found: C 57.55, H 3.98, N 19.62.

General procedure for compounds 10-s Compounds 10-S were obtained starting from $12 \mathbf{a}-\mathbf{d}(0.55 \mathrm{mmol})$ following the same procedure described for $\mathbf{1 a}-\mathbf{j}$. After cooling, the precipitate was recovered by filtration under vacuum.

- 7-Cyclohexylmethyl-5-phenyl-3,7-dihydropyrimido[4,5d]pyridazine-4,8-dione (1o)

Yield $=48 \%(90 \mathrm{mg}) ; \mathrm{mp}=250-253{ }^{\circ} \mathrm{C}(\mathrm{EtOH})$. ${ }^{1} \mathrm{H}-\mathrm{NMR}\left(400 \mathrm{MHz}, \mathrm{DMSO}-\mathrm{d}_{6}\right) \delta$ 0.95-1.25 (m, 5H, $\left.\mathrm{cC}_{6} \mathrm{H}_{11}\right), 1.60-1.70\left(\mathrm{~m}, 5 \mathrm{H}, \mathrm{cC}_{6} \mathrm{H}_{11}\right), 1.85-1.95(\mathrm{~m}, 1 \mathrm{H}$, $\left.\mathrm{cC}_{6} \mathrm{H}_{11}\right), 4.00\left(\mathrm{~d}, 2 \mathrm{H}, \mathrm{NCH}_{2}, J=7.2 \mathrm{~Hz}\right), 7.40(\mathrm{~s}, 5 \mathrm{H}$, Ar), 8.45 (s, 1H, Ar), 13.00 (exch br s, 1H, NH). Anal. Calcd. for $\mathrm{C}_{19} \mathrm{H}_{20} \mathrm{~N}_{4} \mathrm{O}_{2}$ : C 67.84, H 5.99, $\mathrm{N}$ 16.66. Found: C 67.61, H 6.01, N 16.70.

- 7-Benzyl-5-phenyl-3,7-dihydro-pyrimido[4,5-d]pyridazine4,8-dione $(\mathbf{1 p})$

Yield $=65 \%(120 \mathrm{mg}) ; \mathrm{mp}=289-292{ }^{\circ} \mathrm{C}(\mathrm{EtOH})$. ${ }^{1} \mathrm{H}-\mathrm{NMR}\left(400 \mathrm{MHz}, \mathrm{DMSO}-\mathrm{d}_{6}\right) \delta 5.35\left(\mathrm{~s}, 2 \mathrm{H}, \mathrm{NCH}_{2}\right)$, 7.30-7.40 (m, 10H, Ar), 8.45 (s, 1H, Ar), 13.10 (exch br s, $1 \mathrm{H}, \mathrm{NH})$. Anal. Calcd. for $\mathrm{C}_{19} \mathrm{H}_{14} \mathrm{~N}_{4} \mathrm{O}_{2}$ : C 69.08, $\mathrm{H}$ 4.27, N 16.96. Found: C 69.29, H 4.26, N 16.34.

- 7-Cyclopropylmethyl-5-phenyl-3,7-dihydro-pyrimido[4,5d]pyridazine-4,8-dione (1q)

Yield $=32 \%(52 \mathrm{mg}) ; \mathrm{mp}=285-287{ }^{\circ} \mathrm{C}(\mathrm{EtOH})$. ${ }^{1} \mathrm{H}-\mathrm{NMR}\left(400 \mathrm{MHz}, \mathrm{DMSO}-\mathrm{d}_{6}\right) \delta$ 0.35-0.50 (m, 4H, $\left.\mathrm{cC}_{3} \mathrm{H}_{5}\right), 1.25-1.35\left(\mathrm{~m}, 1 \mathrm{H}, \mathrm{cC}_{3} \mathrm{H}_{5}\right), 4.05\left(\mathrm{~d}, 2 \mathrm{H}, \mathrm{NCH}_{2}\right.$, $J=7.2 \mathrm{~Hz}$ ), 7.35-7.45 (m, 5H, Ar), 8.45 (s, 1H, Ar), 13.05 (exch br s, $1 \mathrm{H}, \mathrm{NH}$ ). Anal. Calcd. for $\mathrm{C}_{16} \mathrm{H}_{14} \mathrm{~N}_{4} \mathrm{O}_{2}$ : C 65.30, H 4.79, N 19.04. Found: C 65.49, H 4.80, N 19.09

- 7-(3-Chlorobenzyl)-5-pyridin-3-yl-3,7dihydropyrimido[4,5-d]pyridazine-4,8-dione (1r)

Yield $=15 \%(30 \mathrm{mg}) ; \mathrm{mp}=252-255{ }^{\circ} \mathrm{C}(\mathrm{EtOH}) .{ }^{1} \mathrm{H}-$ NMR (400 MHz, DMSO-d 6 ) $\delta 5.35\left(\mathrm{~s}, 2 \mathrm{H}, \mathrm{NCH}_{2}\right), 7.30$ (d, 1H, Ar), 7.35-7.45 (m, 4H, Ar), 7.85 (d, 1H, Ar), 8.50 (s, 1H, Ar), 8.55-8.65 (m, 2H, Ar). Anal. Calcd. for $\mathrm{C}_{18} \mathrm{H}_{12} \mathrm{ClN}_{5} \mathrm{O}_{2}$ : C 59.11, H 3.31, $\mathrm{N} 19.15$. Found: C 59.24, H 3.31, N 19.08.

- 7-Cyclopropylmethyl-2-ethyl-5-phenyl-3,7-dihydropyrimido[4,5-d]pyridazine-4,8-dione (1 s)

Yield $=25 \%(45 \mathrm{mg}) ; \mathrm{mp}=209-213{ }^{\circ} \mathrm{C} \mathrm{dec} .\left(\mathrm{Et}_{2} \mathrm{O}\right)$. ${ }^{1} \mathrm{H}-\mathrm{NMR}$ (400 MHz, DMSO-d 6 ) $\delta ~ 0.35-0.50$ (m, 4H,
$\left.\mathrm{cC}_{3} \mathrm{H}_{5}\right), 1.25\left(\mathrm{t}, 3 \mathrm{H}, \mathrm{CH}_{2} \mathrm{CH}_{3}, J=7.2 \mathrm{~Hz}\right), 1.25-1.35(\mathrm{~m}$, $\left.1 \mathrm{H}, \mathrm{cC}_{3} \mathrm{H}_{5}\right), 2.70\left(\mathrm{q}, 2 \mathrm{H}, \mathrm{CH}_{2} \mathrm{CH}_{3}, J=7.2 \mathrm{~Hz}\right), 4.00(\mathrm{~d}$, $2 \mathrm{H}, \mathrm{NCH}_{2}, J=7.2 \mathrm{~Hz}$ ), 7.35-7.45 (m, 5H, Ar), 13.05 (exch br s, $1 \mathrm{H}, \mathrm{NH}$ ). Anal. Calcd. for $\mathrm{C}_{18} \mathrm{H}_{18} \mathrm{~N}_{4} \mathrm{O}_{2}$ : C 67.07, H 5.63, N, 17.38. Found: C 67.28, H 5.65, N 17.43 .

General procedure for compounds 1t,u Compounds 1t, were obtained starting from $\mathbf{1 0}$ and $\mathbf{1 q}$ adopting the general procedure described for compounds 7 . For $\mathbf{1 u}$, the mixture was diluted with cold water, extracted with $\mathrm{CH}_{2} \mathrm{Cl}_{2}$ $(3 \times 15 \mathrm{~mL})$ and the solvent was evaporated in vacuo. Lastly, the final compound was purified by flash column chromatography using cyclohexane/ethyl acetate 1:3 as eluent.

- 7-Cyclohexylmethyl-3-ethyl-5-phe nyl-3,7dihydropyrimido[4,5-d]pyridazine-4,8-dione (1t)

Yield $=56 \%(102 \mathrm{mg}) ; \mathrm{mp}=216-219{ }^{\circ} \mathrm{C}(\mathrm{EtOH})$. ${ }^{1} \mathrm{H}-\mathrm{NMR}\left(400 \mathrm{MHz}, \mathrm{CDCl}_{3}\right) \delta 1.05-1.30(\mathrm{~m}, 5 \mathrm{H}$, $\left.\mathrm{cC}_{6} \mathrm{H}_{11}\right), 1.40\left(\mathrm{t}, 3 \mathrm{H}, \mathrm{CH}_{2} \mathrm{CH}_{3}, J=7.2 \mathrm{~Hz}\right), 1.60-1.80$ $\left(\mathrm{m}, 5 \mathrm{H}, \mathrm{cC}_{6} \mathrm{H}_{11}\right), 2.00-2.10\left(\mathrm{~m}, 1 \mathrm{H}, \mathrm{cC}_{6} \mathrm{H}_{11}\right), 4.00(\mathrm{q}$, $\left.2 \mathrm{H}, \mathrm{CH}_{2} \mathrm{CH}_{3}, J=7.2 \mathrm{~Hz}\right), 4.20\left(\mathrm{~d}, 2 \mathrm{H}, \mathrm{CH}_{2} \mathrm{cC}_{6} \mathrm{H}_{11}\right.$, $J=6.8 \mathrm{~Hz}$ ), 7.40-7.50 (m, 5H, Ar), 8.40 (s, 1H, Ar). Anal. Calcd. for $\mathrm{C}_{21} \mathrm{H}_{24} \mathrm{~N}_{4} \mathrm{O}_{2}$ : C 69.21, H 6.64, N 15.37. Found: C 69.38, H 6.62, N 15.33.

- 7-Cyclopropylmethyl-3-ethyl-5-phenyl-3,7dihydropyrimido[4,5-d]pyridazine-4,8-dione (1u)

Yield $=62 \%(100 \mathrm{mg}) ; \mathrm{mp}=176-180{ }^{\circ} \mathrm{C}(\mathrm{EtOH}) .{ }^{1} \mathrm{H}-$ NMR (400 MHz, $\left.\mathrm{CDCl}_{3}\right) \delta 0.45-0.60\left(\mathrm{~m}, 4 \mathrm{H}, \mathrm{cC}_{3} \mathrm{H}_{5}\right)$, $1.40\left(\mathrm{t}, 3 \mathrm{H}, \mathrm{CH}_{2} \mathrm{CH}_{3}, J=7.2 \mathrm{~Hz}\right), 1.40-1.55(\mathrm{~m}, 1 \mathrm{H}$, $\left.\mathrm{cC}_{3} \mathrm{H}_{5}\right), 4.05\left(\mathrm{q}, 2 \mathrm{H}, \mathrm{CH}_{2} \mathrm{CH}_{3}, J=7.2 \mathrm{~Hz}\right), 4.20(\mathrm{~d}, 2 \mathrm{H}$, $\mathrm{CH}_{2} \mathrm{CC}_{3} \mathrm{H}_{5}, \mathrm{~J}=6.8 \mathrm{~Hz}$ ), 7.40-7.55 (m, 5H, Ar), 8.45 (s, $1 \mathrm{H}, \mathrm{Ar}$ ). Anal. Calcd. for $\mathrm{C}_{18} \mathrm{H}_{18} \mathrm{~N}_{4} \mathrm{O}_{2}: \mathrm{C} 67.07, \mathrm{H} 5.63 \mathrm{~N}$ 17.38. Found: C 67.23, H 5.64, N 17.33.

General procedure for compounds $18 \mathrm{~b}$ and $2 \mathrm{a}$ A mixture of the ester 15 [17] (0.06 g, $0.26 \mathrm{mmol}), 0.04 \mathrm{~g}(0.26 \mathrm{mmol})$ of the appropriate R-benzyl chloride and $\mathrm{K}_{2} \mathrm{CO}_{3}(0.07 \mathrm{~g}$, $0.52 \mathrm{mmol})$ in anhydrous DMF $(4 \mathrm{~mL})$ was heated at $50{ }^{\circ} \mathrm{C}$ for $30 \mathrm{~min}$. After cooling, water was added and the mixture was extracted with $\mathrm{CH}_{2} \mathrm{Cl}_{2}(3 \times 15 \mathrm{~mL})$. The organic phase was recovered and dried on sodium sulphate. After removal of the solvent, the residue was purified by flash column chromatography using toluene/ethyl acetate 1:1 as eluent.

- Ethyl-1,4-dihydro-1-(4-chlorobenzyl)-7-methyl-4-oxo1,8-naphtyridine-3-carboxylate $(2 a)$

Yield $=80 \%(75 \mathrm{mg}) ; \mathrm{mp}=139-141{ }^{\circ} \mathrm{C}($ Cyclohexane). ${ }^{1} \mathrm{H}-\mathrm{NMR}\left(400 \mathrm{MHz}, \mathrm{DMSO}-\mathrm{d}_{6}\right) \delta 1.28(\mathrm{t}, 3 \mathrm{H}$, $\left.\mathrm{CH}_{2} \mathrm{CH}_{3}, J=7.0 \mathrm{~Hz}\right), 2.65\left(\mathrm{~s}, 3 \mathrm{H}, \mathrm{CH}_{3}\right), 4.25$ (q, 2H, $\mathrm{CH}_{2} \mathrm{CH}_{3}, \mathrm{~J}=7.0 \mathrm{~Hz}$ ), 5.65 (s, 2H, $\left.\mathrm{CH}_{2}-\mathrm{Ph}\right), 7.35-7.40$ (m, 5H, 1H naphthyridine $\mathrm{H} 6$ and $4 \mathrm{H} \mathrm{Ar}), 8.45(\mathrm{~d}, 1 \mathrm{H}$, naphthyridine $\mathrm{H} 5, J=8.0 \mathrm{~Hz}), 9.00(\mathrm{~s}, 1 \mathrm{H}$, naphtyridine 
$\mathrm{H} 2$ ). Anal. Calcd. for $\mathrm{C}_{19} \mathrm{H}_{17} \mathrm{ClN}_{2} \mathrm{O}_{3}$ : $\mathrm{C} 63.96, \mathrm{H} 4.80, \mathrm{~N}$ 7.85. Found: C 63.78, H 4.79, N 7.87.

- Ethyl-1,4-dihydro-1-(4-fluorobenzyl)-7-methyl-4-oxo1,8-naphtyridine-3-carboxylate (18b)

Yield 75\% (67 mg); $\mathrm{mp}=139-141{ }^{\circ} \mathrm{C}(\mathrm{EtOH})$. ${ }^{1} \mathrm{H}-\mathrm{NMR}\left(400 \mathrm{MHz}, \mathrm{CDCl}_{3}\right) \delta 1.40\left(\mathrm{t}, 3 \mathrm{H}, \mathrm{CH}_{2} \mathrm{CH}_{3}\right.$, $J=7.0 \mathrm{~Hz}), 2.65\left(\mathrm{~s}, 3 \mathrm{H}, \mathrm{CH}_{3}\right), 4.25\left(\mathrm{q}, 2 \mathrm{H}, \mathrm{CH}_{2} \mathrm{CH}_{3}\right.$, $J=7.0 \mathrm{~Hz}), 5.60\left(\mathrm{~s}, 2 \mathrm{H}, \mathrm{CH}_{2}-\mathrm{Ph}\right), 7.05(\mathrm{t}, 2 \mathrm{H}, \mathrm{Ar}$, $J=7.6 \mathrm{~Hz}), 7.25(\mathrm{~d}, 1 \mathrm{H}$, naphtyridine $\mathrm{H} 6, J=8.0 \mathrm{~Hz})$, $7.35(\mathrm{dd}, 2 \mathrm{H}, \mathrm{Ar}, J=8.4 \mathrm{~Hz}$ and $J=1.2 \mathrm{~Hz}), 8.65(\mathrm{~d}, 1 \mathrm{H}$, napthyridine $\mathrm{H} 5, J=8.0 \mathrm{~Hz}), 8.70(\mathrm{~s}, 1 \mathrm{H}$, naphtyridine $\mathrm{H} 2$ ). Anal. Calcd. for $\mathrm{C}_{19} \mathrm{H}_{17} \mathrm{FN}_{2} \mathrm{O}_{3}: \mathrm{C} 67.05, \mathrm{H} 5.03, \mathrm{~N}$ 8.23. Found: C 67.22, H 5.03, N 8.21.

General procedure for compounds $18 \mathrm{c}, \mathrm{d}$ and $\mathbf{2 b}$ A mixture of the ester 15 [17] $(0.11 \mathrm{~g}, 0.50 \mathrm{mmol})$, the appropriate R-phenylboronic acid $(0.120 \mathrm{~g}, 1.0 \mathrm{mmol})$, copper acetate $(0.14 \mathrm{~g}, 0.76 \mathrm{mmol})$ and triethylamine $(0.14 \mathrm{~g}, 0.1 \mathrm{mmol})$ in anhydrous $\mathrm{CH}_{2} \mathrm{Cl}_{2}(2 \mathrm{~mL})$ was stirred at room temperature for $24 \mathrm{~h}$. After dilution with $\mathrm{CH}_{2} \mathrm{Cl}_{2}(10 \mathrm{~mL})$, the suspension was sequentially washed with $33 \%$ aqueous ammonia $(3 \times 10 \mathrm{~mL}), 10 \% \mathrm{NH}_{4} \mathrm{Cl}(1 \times 10 \mathrm{~mL})$ and $\mathrm{H}_{2} \mathrm{O}(2 \times 10 \mathrm{~mL})$. The organic layer was dried and evaporated in vacuo. The crude residue was purified by flash column chromatography using cyclohexane/ethyl acetate 1:2 as eluent.

- Ethyl-1,4-dihydro-7-methyl-4-oxo-1-phenyl1,8-naphtyridine-3-carboxylate (18c)

Yield $=60 \%(93 \mathrm{mg}) ; \mathrm{mp}=202-205{ }^{\circ} \mathrm{C}(\mathrm{EtOH})$. ${ }^{1} \mathrm{H}-\mathrm{NMR}\left(400 \mathrm{MHz}, \mathrm{CDCl}_{3}\right) \delta 1.40\left(\mathrm{t}, 3 \mathrm{H}, \mathrm{CH}_{2} \mathrm{CH}_{3}\right.$, $J=7.2 \mathrm{~Hz}), 2.50\left(\mathrm{~s}, 3 \mathrm{H}, \mathrm{CH}_{3}\right), 4.40\left(\mathrm{q}, 2 \mathrm{H}, \mathrm{CH}_{2} \mathrm{CH}_{3}\right.$, $J=7.2 \mathrm{~Hz}), 7.25-7.35(\mathrm{~m}, 1 \mathrm{H}$, naphthyridine $\mathrm{H} 6)$, 7.45-7.50 (m, 2H, Ar), 7.55-7.60 (m, 3H, Ar), 8.70$8.75(\mathrm{~m}, 2 \mathrm{H}$, naphtyridine $\mathrm{H} 5$ and $\mathrm{H} 6)$. Anal. Calcd. for $\mathrm{C}_{18} \mathrm{H}_{16} \mathrm{~N}_{2} \mathrm{O}_{3}$ : C 70.12, H 5.23, N 9.09. Found: C 70.33, H 5.22, N 9.06.

- Ethyl-1,4-dihydro-1-(3-fluorophenyl)-7-methyl-4-oxo1,8-naphtyridine-3-carboxylate (18d)

Yield $=12 \%(20 \mathrm{mg}) ; \mathrm{mp}=218-221{ }^{\circ} \mathrm{C}(\mathrm{EtOH})$. ${ }^{1} \mathrm{H}-\mathrm{NMR}\left(400 \mathrm{MHz}, \mathrm{CDCl}_{3}\right) \delta 1.40\left(\mathrm{t}, 3 \mathrm{H}, \mathrm{CH}_{2} \mathrm{CH}_{3}\right.$, $J=7.2 \mathrm{~Hz}), 2.55\left(\mathrm{~s}, 3 \mathrm{H}, \mathrm{CH}_{3}\right), 4.45\left(\mathrm{q}, 2 \mathrm{H}, \mathrm{CH}_{2} \mathrm{CH}_{3}\right.$, $J=7.2 \mathrm{~Hz}), 7.30-7.70(\mathrm{~m}, 4 \mathrm{H}$, naphthyridine $\mathrm{H6}$ and $3 \mathrm{H} \mathrm{Ar}), 7.50-7.60$ (m, 1H, Ar), 8.65 (s, 1H, naphthyridine $\mathrm{H} 2), 8.70(\mathrm{~d}, 1 \mathrm{H}$, naphthyridine $\mathrm{H} 5, J=8.0 \mathrm{~Hz}$ ). Anal. Calcd. for $\mathrm{C}_{18} \mathrm{H}_{15} \mathrm{FN}_{2} \mathrm{O}_{3}$ : C 66.25, H 4.63, N 8.58. Found: C 66.41, H 4.64, N 8.55.

- Ethyl 7-methyl-1-(3-nitrophenyl)-4-oxo-1,4-dihydro1,8-naphthyridine-3-carboxylate (2b)

Yield $=45 \%(80 \mathrm{mg}) ; \mathrm{mp}=212-215{ }^{\circ} \mathrm{C}(\mathrm{EtOH})$. ${ }^{1} \mathrm{H}-\mathrm{NMR}\left(400 \mathrm{MHz}, \mathrm{CDCl}_{3}\right) \delta 1.30\left(\mathrm{t}, 3 \mathrm{H}, \mathrm{CH}_{2} \mathrm{CH}_{3}\right.$, $J=7.2 \mathrm{~Hz}), 2.53\left(\mathrm{~s}, 3 \mathrm{H}, \mathrm{CH}_{3}\right), 4.44\left(\mathrm{q}, 2 \mathrm{H}, \mathrm{CH}_{2} \mathrm{CH}_{3}\right.$, $J=7.2 \mathrm{~Hz}), 7.30-7.35(\mathrm{~m}, 1 \mathrm{H}$, naphthyridine $\mathrm{H} 6)$, 7.41 (s, 1H, naphthyridine $\mathrm{H} 2), 7.50-7.60$ (m, 3H, Ar),
8.40-8.50 (m, 2H, Ar), 8.72 (d, 1H, naphthyridine $\mathrm{H} 5$, $J=8.0 \mathrm{~Hz}$ ). Anal. Calcd. for $\mathrm{C}_{18} \mathrm{H}_{15} \mathrm{~N}_{3} \mathrm{O}_{5}: \mathrm{C} 61.19, \mathrm{H}$ 4.28, N 11.89. Found: C 61.37, H 4.29, N 11.83.

General procedure for the synthesis of the carboxylic acids 19b-e Compounds 19b-e were obtained starting from intermediate $\mathbf{2 a}$ and $\mathbf{1 8 b}-\mathbf{d}(1.2 \mathrm{mmol})$ following the procedure previously described for $\mathbf{1} \mathbf{~ m , n}$.

- 1,4-Dihydro-1-(4-chlorobenzyl)-7-methyl-4-oxo1,8-naphtyridine-3-carboxylic acid (19b)

Yield $=95 \%(375 \mathrm{mg}) ; \mathrm{mp}=252-255^{\circ} \mathrm{C}(\mathrm{EtOH}) .{ }^{1} \mathrm{H}-$ NMR (400 MHz, DMSO-d 6 ) $\delta 2.75\left(\mathrm{~s}, 3 \mathrm{H}, \mathrm{CH}_{3}\right), 5.70$ (s, $\left.2 \mathrm{H}, \mathrm{CH}_{2}-\mathrm{Ph}\right), 7.25-7.35$ (m, 4H, Ar), 7.45 (d, 1H, naphtyridine $\mathrm{H} 6, J=8.0 \mathrm{~Hz}), 8.70(\mathrm{~d}, 1 \mathrm{H}$, naphtyridine $\mathrm{H} 5$, $J=8.0 \mathrm{~Hz}$ ), 9.00 (s, $1 \mathrm{H}$, naphtyridine $\mathrm{H} 2$ ), 14.55 (exch br s, $1 \mathrm{H}, \mathrm{COOH}$ ). Anal. Calcd. for $\mathrm{C}_{17} \mathrm{H}_{13} \mathrm{ClN}_{2} \mathrm{O}_{3}: \mathrm{C} 62.11$, H 3.99, N, 8.52. Found: C 62.30, H 3.98, N 8.49.

- 1,4-Dihydro-1-(4-fluorobenzyl)-7-methyl-4-oxo1,8-naphtyridine-3-carboxylic acid $(\mathbf{1 9 c})$

Yield $=97 \%(363 \mathrm{mg}) ; \mathrm{mp}=253-256{ }^{\circ} \mathrm{C}(\mathrm{EtOH}) .{ }^{1} \mathrm{H}-$ NMR $\left(400 \mathrm{MHz}, \mathrm{CDCl}_{3}\right) \delta 2.80\left(\mathrm{~s}, 3 \mathrm{H}, \mathrm{CH}_{3}\right), 5.70(\mathrm{~s}$, $\left.2 \mathrm{H}, \mathrm{CH}_{2}\right), 7.05(\mathrm{t}, 2 \mathrm{H}, \mathrm{Ar}, J=7.6 \mathrm{~Hz}), 7.40-7.50(\mathrm{~m}, 3 \mathrm{H}$, $2 \mathrm{H} \mathrm{Ar}$ and naphtyridine $\mathrm{H} 6), 8.70(\mathrm{~d}, 1 \mathrm{H}$, naphtyridine $\mathrm{H} 5, J=8.0 \mathrm{~Hz}$ ), 9.00 (s, $1 \mathrm{H}$, naphtyridine $\mathrm{H} 2), 14.60$ (exch br s, $1 \mathrm{H}, \mathrm{COOH}$ ). Anal. Calcd. for $\mathrm{C}_{17} \mathrm{H}_{13} \mathrm{FN}_{2} \mathrm{O}_{3}$ : C 65.38, H 4.20, N, 8.97. Found: C 65.13, H 4.21, N 8.95.

- 1,4-Dihydro-1-phenyl-7-methyl-4-oxo-1,8-naphtyridine3-carboxylic acid (19d)

Yield $=90 \%(302 \mathrm{mg}) ; \mathrm{mp}=274-276{ }^{\circ} \mathrm{C}(\mathrm{EtOH}) .{ }^{1} \mathrm{H}-$ NMR (400 MHz, $\left.\mathrm{CDCl}_{3}\right) \delta 2.60\left(\mathrm{~s}, 3 \mathrm{H}, \mathrm{CH}_{3}\right), 7.40-7.50$ (m, 3H, 2H Ar and naphtyridine H6), 7.55-7.65 (m, 3H, Ar), $8.75(\mathrm{~d}, 1 \mathrm{H}$, naphtyridine $\mathrm{H} 5, J=8.0 \mathrm{~Hz}), 9.00$ (s, $1 \mathrm{H}$, naphtyridine $\mathrm{H} 2$ ), 14.55 (exch br s, $1 \mathrm{H}, \mathrm{COOH}$ ). Anal. Calcd. for $\mathrm{C}_{16} \mathrm{H}_{12} \mathrm{~N}_{2} \mathrm{O}_{3}$ : C 68.56, H 4.32, N 9.99. Found: C 68.41, H 4.33, N 9.94.

- 1,4-Dihydro-1-(3-fluorophenyl)-7-methyl-4-oxo1,8-naphtyridine-3-carboxylic acid $(19 e)$

Yield $=98 \%(350 \mathrm{mg}) ; \mathrm{mp}>300{ }^{\circ} \mathrm{C}(\mathrm{EtOH}) .{ }^{1} \mathrm{H}-$ NMR (400 MHz, $\left.\mathrm{CDCl}_{3}\right) \delta 2.65$ (s, 3H, $\left.\mathrm{CH}_{3}\right), 7.20-7.30$ (m, 3H, Ar), $7.40(\mathrm{~d}, 1 \mathrm{H}$, naphtyridine $\mathrm{H6}, J=8.0 \mathrm{~Hz}$ ), 7.50-7.60 (m, 1H, Ar), $8.70(\mathrm{~d}, 1 \mathrm{H}$, naphtyridine $\mathrm{H} 5$, $J=8.0 \mathrm{~Hz}$ ), 9.00 (s, $1 \mathrm{H}$, naphtyridine $\mathrm{H} 2), 14.40$ (exch br s, $1 \mathrm{H}, \mathrm{COOH}$ ). Anal. Calcd. for $\mathrm{C}_{16} \mathrm{H}_{11} \mathrm{FN}_{2} \mathrm{O}_{3}: \mathrm{C} 64.43$, H 3.72, N, 9.39 Found: C 64.22, H 3.71, N 9.36.

General procedure for the synthesis of final compounds $2 \mathrm{c}-\mathrm{i}$ The appropriate carboxylic acid of type $19(0.24 \mathrm{mmol})$ was converted into the acyl chloride with $\mathrm{SOCl}_{2}(6.5 \mathrm{mmol})$ and triethylamine $(0.06 \mathrm{~mL})$ at room temperature for 40-60 min. After removing the excess $\mathrm{SOCl}_{2}$, the residue was washed 3 times with cyclohexane and used in the following step without further purification. Under stirring at 
$0{ }^{\circ} \mathrm{C}$, a solution of the appropriate acyl chloride $(1.3 \mathrm{mmol})$ in anhydrous THF (5-7 mL) was added dropwise to a solution of the appropriate amine $(2.6 \mathrm{mmol})$ dissolved in anhydrous THF (3-7 mL). The mixture was stirred at room temperature for $2-3 \mathrm{~h}$. After removed of the solvent, the residue was treated with cold water and extracted with $\mathrm{CH}_{2} \mathrm{Cl}_{2}$ $(3 \times 15 \mathrm{~mL})$. The crude amides were purified by flash column chromatography using $\mathrm{CHCl}_{3} / \mathrm{MeOH}$ 9:1 (for 2d,e), cyclohexane/ethyl acetate 1:2 (2f), EtOH/CHCl ${ }_{3}$ /ethyl ether/ petroleum ether/33\% $\mathrm{NH}_{4} \mathrm{OH}$ 4.5:18:18:45:0.25 (for $\mathbf{2} \mathbf{~ g , h}$ ),

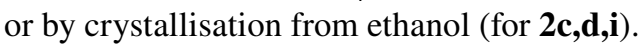

- 1-Ethyl-7-methyl-4-oxo-N-(pyridin-3-yl)-1,4-dihydro1,8-naphthyridine-3-carboxamide (2c)

Yield $=10 \%(10 \mathrm{mg}) ; \mathrm{mp}=160-163{ }^{\circ} \mathrm{C}(\mathrm{EtOH})$. ${ }^{1} \mathrm{H}-\mathrm{NMR}\left(400 \mathrm{MHz}, \mathrm{CDCl}_{3}\right) \delta 1.60\left(\mathrm{t}, 3 \mathrm{H}, \mathrm{CH}_{2} \mathrm{CH}_{3}\right.$, $J=7.0 \mathrm{~Hz}), 2.75\left(\mathrm{~s}, 3 \mathrm{H}, \mathrm{CH}_{3}\right), 4.60\left(\mathrm{q}, 2 \mathrm{H}, \mathrm{CH}_{2} \mathrm{CH}_{3}\right.$, $J=7.0 \mathrm{~Hz}), 7.30-7.35(\mathrm{~m}, 1 \mathrm{H}$, pyridine $), 7.40(\mathrm{~d}, 1 \mathrm{H}$, naphthyridine $\mathrm{H} 6, J=8.0 \mathrm{~Hz}), 8.25-8.30(\mathrm{~m}, 1 \mathrm{H}$, pyridine), $8.30-8.40(\mathrm{~m}, 1 \mathrm{H}$, pyridine $), 8.70(\mathrm{~d}, 1 \mathrm{H}$, naphthyridine $\mathrm{H} 5, J=8.0 \mathrm{~Hz}), 8.95(\mathrm{~s}, 1 \mathrm{H}$, pyridine), $9.05(\mathrm{~s}$, $1 \mathrm{H}$, naphthyridine $\mathrm{H} 2$ ), 12.35 (exch br s, $1 \mathrm{H}, \mathrm{NH}$ ). Anal. Calcd. for $\mathrm{C}_{17} \mathrm{H}_{16} \mathrm{~N}_{4} \mathrm{O}_{2}:$ C 66.22, H 5.23, N 18.17. Found: C 66.06, H 5.25, N 18.12 .

- $N^{\prime}$-Acetyl-1-ethyl-7-methyl-4-oxo-1,4-dihydro1,8-naphthyridine-3-carbohydrazide $(\mathbf{2 d})$

Yield $=15 \%(10 \mathrm{mg}) ; \mathrm{mp}=264-267{ }^{\circ} \mathrm{C}(\mathrm{EtOH})$. ${ }^{1} \mathrm{H}-\mathrm{NMR}\left(400 \mathrm{MHz}, \mathrm{CDCl}_{3}\right) \delta 1.55\left(\mathrm{t}, 3 \mathrm{H}, \mathrm{CH}_{2} \mathrm{CH}_{3}\right.$, $J=7.0 \mathrm{~Hz}), 2.20\left(\mathrm{~s}, 3 \mathrm{H}, \mathrm{COCH}_{3}\right), 2.70\left(\mathrm{~s}, 3 \mathrm{H}, \mathrm{CH}_{3}\right), 4.60$ (q, $\left.2 \mathrm{H}, \mathrm{CH}_{2} \mathrm{CH}_{3}, J=7.0 \mathrm{~Hz}\right), 7.35(\mathrm{~d}, 1 \mathrm{H}$, naphthyridine H6, $J=8.4 \mathrm{~Hz}$ ), 8.05 (exch br s, $1 \mathrm{H}, \mathrm{NH}), 8.70$ (d, 1H, naphthyridine $\mathrm{H} 5, J=8.0 \mathrm{~Hz}$ ), 9.00 (s, $1 \mathrm{H}$, naphthyridine H2), 12.10 (exch br s, 1H, NH). Anal. Calcd. for $\mathrm{C}_{14} \mathrm{H}_{16} \mathrm{~N}_{4} \mathrm{O}_{3}:$ C 58.32, H 5.59, N 19.43. Found: C 58.47, H 5.60, N 19.48 .

- 1-Ethyl-N,7-dimethyl-4-oxo-N-phenyl-1,4-dihydro1,8-naphthyridine-3-carboxamide (2e)

Yield $=63 \%(50 \mathrm{mg}) ; \mathrm{mp}=160-163{ }^{\circ} \mathrm{C}(\mathrm{EtOH})$. ${ }^{1} \mathrm{H}-\mathrm{NMR}\left(400 \mathrm{MHz}, \mathrm{CDCl}_{3}\right) \delta 1.40\left(\mathrm{t}, 3 \mathrm{H}, \mathrm{CH}_{2} \mathrm{CH}_{3}\right.$, $J=7.2 \mathrm{~Hz}), 2.60\left(\mathrm{~s}, 3 \mathrm{H}, \mathrm{CH}_{3}\right), 3.50\left(\mathrm{~s}, 3 \mathrm{H}, \mathrm{N}-\mathrm{CH}_{3}\right), 4.40$ (q, $\left.2 \mathrm{H}, \mathrm{CH}_{2} \mathrm{CH}_{3}, J=7.2 \mathrm{~Hz}\right), 7.10-7.15(\mathrm{~m}, 2 \mathrm{H}, \mathrm{Ar})$, 7.15-7.30 (m, 4H, 3H Ar and naphthyridine H6), 7.90$8.00(\mathrm{~m}, 1 \mathrm{H}$, naphthyridine $\mathrm{H} 5), 8.40-8.50(\mathrm{~m}, 1 \mathrm{H}$, naphthyridine $\mathrm{H} 2$ ). Anal. Calcd. for $\mathrm{C}_{19} \mathrm{H}_{19} \mathrm{~N}_{3} \mathrm{O}_{2}: \mathrm{C} 71.01, \mathrm{H}$ 5.96, N 13.08. Found: C 71.17, H 5.95, N 13.12.

- 1-Ethyl-7-methyl-N-(naphthalen-2-yl)-4-oxo-1,4-dihydro1,8-naphthyridine-3-carboxamide (2f)

Yield $=60 \%(50 \mathrm{mg}) ; \mathrm{mp}=217-220{ }^{\circ} \mathrm{C}(\mathrm{EtOH})$. ${ }^{1} \mathrm{H}-\mathrm{NMR}\left(400 \mathrm{MHz}, \mathrm{CDCl}_{3}\right) \delta 1.60\left(\mathrm{t}, 3 \mathrm{H}, \mathrm{CH}_{2} \mathrm{CH}_{3}\right.$, $J=7.0 \mathrm{~Hz}), 2.80\left(\mathrm{~s}, 3 \mathrm{H}, \mathrm{CH}_{3}\right), 4.65\left(\mathrm{q}, 2 \mathrm{H}, \mathrm{CH}_{2} \mathrm{CH}_{3}\right.$, $J=7.0 \mathrm{~Hz}), 7.40(\mathrm{~d}, 1 \mathrm{H}$, naphthyridine $\mathrm{H} 6, J=8.4 \mathrm{~Hz}$ ), 7.55 (t, 2H, Ar, J=7.6 Hz), 7.60-7.70 (m, 2H, Ar), 7.90 $(\mathrm{d}, 1 \mathrm{H}, \mathrm{Ar}, J=7.6 \mathrm{~Hz}), 8.40$ (d, 1H, Ar, $J=8.0 \mathrm{~Hz}), 8.55$ (d, 1H, Ar, $J=7.8 \mathrm{~Hz}), 8.80$ (d, 1H, naphthyridine $\mathrm{H} 5$, $J=8.0 \mathrm{~Hz}$ ), 9.15 (s, $1 \mathrm{H}$, naphthyridine $\mathrm{H} 2$ ), 12.80 (exch br s, $1 \mathrm{H}, \mathrm{NH}$ ). Anal. Calcd. for $\mathrm{C}_{22} \mathrm{H}_{19} \mathrm{~N}_{3} \mathrm{O}_{2}: \mathrm{C} 73.93, \mathrm{H}$ 5.36, N, 11.76. Found: C 73.70, H 5.34, N 11.73.

- 1-(4-Chlorobenzyl)-N,7-dimethyl-4-oxo-1,4-dihydro1,8-naphthyridine-3-carboxamide (2 $\mathrm{g}$ )

Yield $=20 \%(16 \mathrm{mg}) ; \mathrm{mp}=231-233{ }^{\circ} \mathrm{C}(\mathrm{EtOH}) .{ }^{1} \mathrm{H}-$ NMR $\left(400 \mathrm{MHz}, \mathrm{CDCl}_{3}\right) \delta 2.70\left(\mathrm{~s}, 3 \mathrm{H}, \mathrm{CH}_{3}\right), 3.05(\mathrm{~d}$, $\left.3 \mathrm{H}, \mathrm{NH}-\mathrm{CH}_{3}, J=9.2 \mathrm{~Hz}\right), 5.65\left(\mathrm{~s}, 2 \mathrm{H}, \mathrm{CH}_{2}-\mathrm{Ph}\right), 7.30$ $7.40(\mathrm{~m}, 5 \mathrm{H}, 4 \mathrm{H} \mathrm{Ar}$ and naphthyridine $\mathrm{H6}), 8.66(\mathrm{~d}$, $1 \mathrm{H}$, naphthyridine $\mathrm{H} 5, J=8.0 \mathrm{~Hz}), 9.00$ (s, $1 \mathrm{H}$, naphthyridine $\mathrm{H} 2$ ), 9.75 (exch br s, 1H, NH). Anal. Calcd. for $\mathrm{C}_{18} \mathrm{H}_{16} \mathrm{ClN}_{3} \mathrm{O}_{2}$ : C 63.25, H 4.72, N 12.29. Found: $\mathrm{C}$ 63.02, H 4.73, N 12.33 .

- 1-(3-Fluorophenyl)-N,7-dimethyl-4-oxo-1,4-dihydro1,8-naphthyridine-3-carboxamide $(\mathbf{2} \boldsymbol{h})$

Yield $=70 \%(52 \mathrm{mg}) ; \mathrm{mp}=201-204{ }^{\circ} \mathrm{C}(\mathrm{EtOH}) .{ }^{1} \mathrm{H}-$ NMR (400 MHz, $\left.\mathrm{CDCl}_{3}\right) \delta 2.60\left(\mathrm{~s}, 3 \mathrm{H}, \mathrm{CH}_{3}\right), 3.05(\mathrm{~d}$, $3 \mathrm{H}, \mathrm{NH}-\mathrm{CH}_{3}, J=6.0 \mathrm{~Hz}$ ), 7.20-7.30 (m, 3H, Ar), 7.35 (d, $1 \mathrm{H}$, naphtyridine $\mathrm{H} 6, J=8.0 \mathrm{~Hz}), 7.50(\mathrm{t}, 1 \mathrm{H}, \mathrm{Ar}$, $J=7.6 \mathrm{~Hz}), 8.70(\mathrm{~d}, 1 \mathrm{H}$, naphtyridine $\mathrm{H} 5, J=8.0 \mathrm{~Hz})$, 9.00 (s, $1 \mathrm{H}$, naphtyridine $\mathrm{H} 2), 9.70$ (exch br s, $1 \mathrm{H}, \mathrm{NH}$ ). Anal. Calcd. for $\mathrm{C}_{17} \mathrm{H}_{14} \mathrm{FN}_{3} \mathrm{O}_{2}$ : C 65.59, H 4.53, N 13.50. Found: C 64.45, H 4.55, N 13.53.

- $\quad N^{\prime}$-Acetyl-1-(3-fluorophenyl)-7-methyl-4-oxo-1,4-dihydro1,8-naphthyridine-3-carbohydrazide (2i)

Yield $=87 \%(80 \mathrm{mg}) ; \mathrm{mp}=290-293{ }^{\circ} \mathrm{C}(\mathrm{EtOH})$. ${ }^{1} \mathrm{H}-\mathrm{NMR}\left(400 \mathrm{MHz}, \mathrm{CDCl}_{3}\right) \delta 2.15\left(\mathrm{~s}, 3 \mathrm{H}, \mathrm{COCH}_{3}\right)$, $2.58\left(\mathrm{~s}, 3 \mathrm{H}, \mathrm{CH}_{3}\right), 7.20-7.30(\mathrm{~m}, 3 \mathrm{H}, \mathrm{Ar}), 7.36(\mathrm{~d}, 1 \mathrm{H}$, naphthyridine $\mathrm{H} 6, J=8.0 \mathrm{~Hz}), 7.55-7.60(\mathrm{~m}, 1 \mathrm{H}, \mathrm{Ar})$, 8.55 (exch br s, $1 \mathrm{H}, \mathrm{NH}), 8.73$ (d, $1 \mathrm{H}$, naphthyridine $\mathrm{H} 5$, $J=8.0 \mathrm{~Hz}$ ), 8.90 (s, $1 \mathrm{H}$, naphthyridine $\mathrm{H} 2), 12.15$ (exch br s, $1 \mathrm{H}, \mathrm{NH})$. Anal. Calcd. for $\mathrm{C}_{18} \mathrm{H}_{15} \mathrm{FN}_{4} \mathrm{O}_{3}: \mathrm{C}$ 61.01, H, 4.27, N, 15.81. Found: C 61.15, H 4.28, N 15.86.

General procedure for the synthesis of the 3,5dichloropyridilamides $2 \mathrm{j}, \mathbf{k} \mathrm{NaH}(0.43 \mathrm{mmol})$ was added to a cooled $\left(0^{\circ} \mathrm{C}\right)$ solution of 3,5-dichloro-4-pyridilamine $(0.43 \mathrm{mmol})$ in anhydrous THF ( $3 \mathrm{~mL}$ ), and the mixture was stirred for $3 \mathrm{~h}$. The appropriate acyl chloride $(0.43 \mathrm{mmol})$ in anhydrous THF $(3 \mathrm{~mL})$ was added at $0{ }^{\circ} \mathrm{C}$. After $30 \mathrm{~min}$, the solvent was removed, and the residue was sequentially washed with $6 \mathrm{M} \mathrm{NaOH}(2 \times 10 \mathrm{~mL}), 2 \mathrm{~N} \mathrm{HCl}(2 \times 10 \mathrm{~mL})$ and $\mathrm{H}_{2} \mathrm{O}$. The solid residue was purified by flash column chromatography using cyclohexane/ethyl acetate 2:1 as eluent.

- $N$-(3,5-Dichloropyridin-4-yl)-1-ethyl-7-methyl-4-oxo1,4-dihydro-1,8-naphthyridine-3-carboxamide (2j)

Yield $=7 \%(10 \mathrm{mg}) ; \mathrm{mp}=237-241{ }^{\circ} \mathrm{C}(\mathrm{EtOH})$. ${ }^{1} \mathrm{H}-\mathrm{NMR}\left(400 \mathrm{MHz}, \mathrm{CDCl}_{3}\right) \delta 1.60\left(\mathrm{t}, 3 \mathrm{H}, \mathrm{CH}_{2} \mathrm{CH}_{3}\right.$, $J=7.2 \mathrm{~Hz}), 2.75\left(\mathrm{~s}, 3 \mathrm{H}, \mathrm{CH}_{3}\right), 4.63\left(\mathrm{q}, 2 \mathrm{H}, \mathrm{CH}_{2} \mathrm{CH}_{3}\right.$, $J=7.2 \mathrm{~Hz}), 7.39(\mathrm{~d}, 1 \mathrm{H}$, naphtyridine $\mathrm{H} 6, J=8.0 \mathrm{~Hz})$, $8.60(\mathrm{~s}, 2 \mathrm{H}$, pyridine), 8.75 (d, $1 \mathrm{H}$, naphtyridine $\mathrm{H}$, 
$J=8.4 \mathrm{~Hz}$ ), 9.00 (s, 1H, naphtyridine $\mathrm{H} 2$ ), 12.30 (exch br s, $1 \mathrm{H}, \mathrm{NH})$. Anal. Calcd. for $\mathrm{C}_{17} \mathrm{H}_{14} \mathrm{Cl}_{2} \mathrm{~N}_{4} \mathrm{O}_{2}: \mathrm{C} 54.13$, H 3.74, N 14.85. Found: C 54.19, H 3.75, N 14.88.

- $\quad$-(3,5-Dichloropyridin-4-yl)-1-(4-fluorobenzyl)-7-methyl4-oxo-1,4-dihydro-1,8-naphthyridine-3-carboxamide ( $\mathbf{k})$

Yield $=7 \%(14 \mathrm{mg}) ; \mathrm{mp}=266-269^{\circ} \mathrm{C}(\mathrm{EtOH}) .{ }^{1} \mathrm{H}-\mathrm{NMR}$ $\left(400 \mathrm{MHz}, \mathrm{CDCl}_{3}\right) \delta 2.76\left(\mathrm{~s}, 3 \mathrm{H}, \mathrm{CH}_{3}\right), 5.71\left(\mathrm{~s}, 2 \mathrm{H}, \mathrm{CH}_{2}\right.$ $\mathrm{Ph}), 7.06$ (t, $2 \mathrm{H}, \mathrm{Ar}, J=8.4 \mathrm{~Hz}), 7.40-7.50$ (m, 3H, $2 \mathrm{H} \mathrm{Ar}$ and naphtyridine $\mathrm{H} 6), 8.60$ (s, $2 \mathrm{H}$, pyridine), $8.75(\mathrm{~d}, 1 \mathrm{H}$, naphtyridine $\mathrm{H} 5, J=8.4 \mathrm{~Hz}$ ), 9.10 (s, $1 \mathrm{H}$, naphtyridine $\mathrm{H} 2$ ), 12.20 (exch br s, $1 \mathrm{H}, \mathrm{NH}$ ). Anal. Calcd. for $\mathrm{C}_{22} \mathrm{H}_{15} \mathrm{Cl}_{2} \mathrm{FN}_{4} \mathrm{O}_{2}$ : C 57.78, H 3.31, N, 12.25. Found: C 57.91, H 3.30, N 12.29.

- 1-Ethyl-7-methyl-3-(5-methyl-1,3,4-oxadiazol-2-yl)-1,8naphthyridin-4(1H)-one (2l)

The reaction was performed using $\mathrm{POCl}_{3}$ on silica gel as dehydrating agent. This reagent was prepared as follows: $\mathrm{POCl}_{3}(1.5 \mathrm{~mL})$ was dissolved in anhydrous pyridine ( $3 \mathrm{~mL})$, and the solution was diluted with $\mathrm{CH}_{2} \mathrm{Cl}_{2}$ $(25 \mathrm{~mL})$, then cooled at $0{ }^{\circ} \mathrm{C}$, and the silica gel $(10 \mathrm{~g})$ was added portion-wise. After $1 \mathrm{~h}$, the solvent was carefully removed in vacuo. $0.5 \mathrm{~g}$ di $\mathrm{POCl}_{3}$ adsorbed on silica gel were added to compound $\mathbf{2 d}(0.02 \mathrm{~g}, 0.07 \mathrm{mmol})$ suspended in toluene $(1.5 \mathrm{~mL})$, and the mixture was warmed at $80{ }^{\circ} \mathrm{C}$ for $5 \mathrm{~h}$. The solvent was removed in vacuo, and the residue was extracted 5 times with $\mathrm{CH}_{2} \mathrm{Cl}_{2}$. Evaporation of the solvent afforded the crude product which was purified by flash column chromatography using $\mathrm{CHCl}_{3} /$ $\mathrm{MeOH} 9: 1$ as eluent. Yield $=68 \%(13 \mathrm{mg}) ; \mathrm{mp}=188-$ $191{ }^{\circ} \mathrm{C}(\mathrm{EtOH}) .{ }^{1} \mathrm{H}-\mathrm{NMR}\left(400 \mathrm{MHz}, \mathrm{CDCl}_{3}\right) \delta 1.55(\mathrm{t}$, $3 \mathrm{H}, \mathrm{CH}_{2} \mathrm{CH}_{3}, J=7.0 \mathrm{~Hz}$ ), 2.65 (s, $3 \mathrm{H}, \mathrm{CH}_{3}$ oxadiazole), $2.70\left(\mathrm{~s}, 3 \mathrm{H}, \mathrm{CH}_{3}\right), 4.95$ (q, $2 \mathrm{H}, \mathrm{CH}_{2} \mathrm{CH}_{3}, J=7.0 \mathrm{~Hz}$ ), 7.30 (d, $1 \mathrm{H}$, naphthyridine $\mathrm{H} 6, J=8.0 \mathrm{~Hz}$ ), 8.65-8.70 ( $\mathrm{m}, 2 \mathrm{H}$, naphthyridine $\mathrm{H} 5$ and $\mathrm{H} 2$ ). Anal. Calcd. for $\mathrm{C}_{14} \mathrm{H}_{14} \mathrm{~N}_{4} \mathrm{O}_{2}$ : C 62.21, H 5.22, N 20.73. Found: C 62.08, H 5.23, N 20.80 .

- Ethyl-1,4-dihydro-1-ethyl-7-(3-nitrophenyl)-4-oxo1,8-naphthyridine-3-carboxylate ( $2 \mathrm{~m}$ )

A suspension of 3-nitrophenylboronic acid $(0.06 \mathrm{~g}$, $0.35 \mathrm{mmol}), 2 \mathrm{M} \mathrm{Na}_{2} \mathrm{CO}_{3}(2 \mathrm{~mL})$ in EtOH $(2 \mathrm{~mL})$ was added to a stirred mixture of the ester 20c [23] $(0.10 \mathrm{~g}, 0.35 \mathrm{mmol})$ and tetrakis(triphenylphosphine)palladium $(0)(0.020 \mathrm{~g}$, catalytic amount $)$ in toluene $(2 \mathrm{~mL})$. The reaction was heated at $60{ }^{\circ} \mathrm{C}$ for $90 \mathrm{~min}$. After cooling, the mixture was extracted with toluene $(3 \times 15 \mathrm{~mL})$ and dried on $\mathrm{Na}_{2} \mathrm{SO}_{4}$. The solvent was removed in vacuo, and the residue was purified by flash column chromatography using EtOH/ $\mathrm{CHCl}_{3}$ /ethyl ether/ petroleum ether/33\% $\mathrm{NH}_{4} \mathrm{OH}$ 4.5:18:18:45:0.25 as eluent. Yield $=10 \%(12 \mathrm{mg}) ; \mathrm{mp}=225-228{ }^{\circ} \mathrm{C}(\mathrm{EtOH})$. ${ }^{1} \mathrm{H}-\mathrm{NMR}\left(400 \mathrm{MHz}, \mathrm{CDCl}_{3}\right) \delta 1.45\left(\mathrm{t}, 3 \mathrm{H}, \mathrm{OCH}_{2} \mathrm{CH}_{3}\right.$, $J=7.2 \mathrm{~Hz}), 1.65\left(\mathrm{t}, 3 \mathrm{H}, \mathrm{N}-\mathrm{CH}_{2} \mathrm{CH}_{3}, J=7.2 \mathrm{~Hz}\right), 4.45$ (q, $\left.2 \mathrm{H}, \mathrm{OCH}_{2} \mathrm{CH}_{3}, \mathrm{~J}=7.2 \mathrm{~Hz}\right), 4.65\left(\mathrm{q}, 2 \mathrm{H}, \mathrm{N}-\mathrm{CH}_{2} \mathrm{CH}_{3}\right.$, $J=7.2 \mathrm{~Hz}), 7.76(\mathrm{t}, 1 \mathrm{H}, \mathrm{Ar}, J=8.0 \mathrm{~Hz}), 7.95(\mathrm{~d}, 1 \mathrm{H}$, naph- thyridine $\mathrm{H} 6, J=8.4 \mathrm{~Hz}), 8.40(\mathrm{~d}, 1 \mathrm{H}, \mathrm{Ar}, J=8.0 \mathrm{~Hz})$, $8.48(\mathrm{~d}, 1 \mathrm{H}, \mathrm{Ar}, J=8.0 \mathrm{~Hz}), 8.70(\mathrm{~s}, 1 \mathrm{H}$, naphthyridine $\mathrm{H} 2), 8.90(\mathrm{~d}, 1 \mathrm{H}$, naphthyridine $\mathrm{H} 5, J=8.0 \mathrm{~Hz}), 9.00$ (s, $1 \mathrm{H}, \mathrm{Ar}$ ). Anal. Calcd. for $\mathrm{C}_{19} \mathrm{H}_{17} \mathrm{~N}_{3} \mathrm{O}_{5}: \mathrm{C} 62.12, \mathrm{H} 4.66$, N, 11.44. Found: C 62.26, H 4.64, N 11.41.

General procedure for the synthesis of final compounds 2n-p Compounds $\mathbf{2 n - p}$ were obtained by adopting the same procedure for carboxamides $\mathbf{2 c - i}$, reacting $\mathbf{2 2 a}, \mathbf{b}$ with the appropriate amine. The crude compounds $\mathbf{2 0}, \mathbf{p}$ were purified by flash column chromatography using $\mathrm{EtOH} / \mathrm{CHCl}_{3} /$ ethyl ether/petroleum ether/33\% $\mathrm{NH}_{4} \mathrm{OH}$ 4.5:18:18:45:0.25 as eluent, while compound $\mathbf{2 n}$ was purified by crystallisation from ethanol.

- 1-Ethyl-7-methoxy-4-oxo-N-phenyl-1,4-dihydro1,8-naphthyridine-3-carboxamide (2n)

Yield $=50 \%(38 \mathrm{mg}) ; \mathrm{mp}=189-192{ }^{\circ} \mathrm{C}(\mathrm{EtOH})$. ${ }^{1} \mathrm{H}-\mathrm{NMR}\left(400 \mathrm{MHz}, \mathrm{CDCl}_{3}\right) \delta 1.60\left(\mathrm{t}, 3 \mathrm{H}, \mathrm{CH}_{2} \mathrm{CH}_{3}\right.$, $J=7.2 \mathrm{~Hz}), 4.10\left(\mathrm{~s}, 3 \mathrm{H}, \mathrm{OCH}_{3}\right), 4.53\left(\mathrm{q}, 2 \mathrm{H}, \mathrm{CH}_{2} \mathrm{CH}_{3}\right.$, $J=7.2 \mathrm{~Hz}), 6.91(\mathrm{~d}, 1 \mathrm{H}$, naphthyridine $\mathrm{H} 6, J=8.8 \mathrm{~Hz})$, 7.13 (t, $1 \mathrm{H}, \mathrm{Ar}, J=7.2 \mathrm{~Hz}), 7.38$ (t, $2 \mathrm{H}, \mathrm{Ar}, J=7.8 \mathrm{~Hz}$ ), $7.79(\mathrm{~d}, 2 \mathrm{H}, \mathrm{Ar}, J=8.0 \mathrm{~Hz}) 8.66(\mathrm{~d}, 1 \mathrm{H}$, naphthyridine $\mathrm{H} 5, J=8.4 \mathrm{~Hz}$ ), 8.93 (s, $1 \mathrm{H}$, naphthyridine $\mathrm{H} 2$ ), 12.25 (exch br s, $1 \mathrm{H}, \mathrm{NH}$ ). Anal. Calcd. for $\mathrm{C}_{18} \mathrm{H}_{17} \mathrm{~N}_{3} \mathrm{O}_{3}$ : C 66.86, H 5.30, N, 13.00. Found: C 66.71, H 5.28, N 13.04.

- 1-Ethyl-4-oxo-N-phenyl-7-(pyrrolidin-1-yl)-1,4-dihydro1,8-naphthyridine-3-carboxamide (2o)

Yield $=20 \%(17 \mathrm{mg}) ; \mathrm{mp}=234-237{ }^{\circ} \mathrm{C}(\mathrm{EtOH})$. ${ }^{1} \mathrm{H}-\mathrm{NMR}\left(400 \mathrm{MHz}, \mathrm{CDCl}_{3}\right) \delta 1.50\left(\mathrm{t}, 3 \mathrm{H}, \mathrm{CH}_{2} \mathrm{CH}_{3}\right.$, $J=7.0 \mathrm{~Hz}), 1.50-1.60(\mathrm{~m}, 2 \mathrm{H}$, pyrrolidine), $1.70-1.90$ (m, $4 \mathrm{H}$, pyrrolidine), $2.10-2.20(\mathrm{~m}, 2 \mathrm{H}$, pyrrolidine), 4.40 (q, $\left.2 \mathrm{H}, \mathrm{CH}_{2} \mathrm{CH}_{3}, \mathrm{~J}=7.0 \mathrm{~Hz}\right), 6.50$ (d, 1H, naphthyridine $\mathrm{H} 6$ ), $7.10(\mathrm{t}, 1 \mathrm{H}, \mathrm{Ar}, J=7.2 \mathrm{~Hz}), 7.36(\mathrm{t}, 2 \mathrm{H}, \mathrm{Ar}, J=7.8 \mathrm{~Hz})$, 7.79 (d, $2 \mathrm{H}, \mathrm{Ar}, J=8.4 \mathrm{~Hz}), 8.41$ (d, $1 \mathrm{H}$, naphthyridine $\mathrm{H} 5, J=8.8 \mathrm{~Hz}$ ), 8.80 (s, $1 \mathrm{H}$, naphthyridine $\mathrm{H} 2$ ), 12.40 (exch br s, $1 \mathrm{H}, \mathrm{NH}$ ). Anal. Calcd. for $\mathrm{C}_{21} \mathrm{H}_{22} \mathrm{~N}_{4} \mathrm{O}_{2}$ : C 69.59, H 6.12, N 15.46. Found: C 69.78, H 6.10, N 15.51.

- 1-Ethyl-N-methyl-4-oxo-N-phenyl-7-(pyrrolidin-1-yl)1,4-dihydro-1,8-naphthyridine-3-carboxamide (2p)

Yield $=50 \%$ (45 mg); oil. ${ }^{1} \mathrm{H}-\mathrm{NMR}\left(400 \mathrm{MHz}, \mathrm{CDCl}_{3}\right) \delta$ $1.35\left(\mathrm{t}, 3 \mathrm{H}, \mathrm{CH}_{2} \mathrm{CH}_{3}\right), 1.50-1.60$ (m, $2 \mathrm{H}$, pyrrolidine), 1.60 1.75 (m, $2 \mathrm{H}$, pyrrolidine), $1.75-1.80$ (m, $2 \mathrm{H}$, pyrrolidine), 2.00-2.15 (m, $2 \mathrm{H}$, pyrrolidine), 3.50 (s, $\left.3 \mathrm{H}, \mathrm{N}-\mathrm{CH}_{3}\right), 4.20$ (q, $2 \mathrm{H}, \mathrm{CH}_{2} \mathrm{CH}_{3}$ ), 6.40 (d, $1 \mathrm{H}$, naphthyridine $\mathrm{H} 6$ ), 7.10-7.20 (m, 1H, Ar), 7.15 (s, 1H, naphthyridine $\mathrm{H} 2), 7.20-7.30$ (m, $4 \mathrm{H}, \mathrm{Ar}), 8.15$ (d,1H, naphthyridine H5). Anal. Calcd. for $\mathrm{C}_{22} \mathrm{H}_{24} \mathrm{~N}_{4} \mathrm{O}_{2}: \mathrm{C} 70.19, \mathrm{H} 6.43, \mathrm{~N} 14.88$. Found: $\mathrm{C} 70.03, \mathrm{H}$ $6.45, \mathrm{~N} 14.83$.

- 6-Methyl-4-oxo-4H-pyrido[1,2-a]pyrimidine-3-carboxylic acid (17)

A suspension of the ethyl ester 16 [24] (0.085 mmol) in a mixture of acetic acid $(5 \mathrm{~mL})$ and $6 \mathrm{M} \mathrm{HCl}(5 \mathrm{~mL})$ was refluxed for $3 \mathrm{~h}$. After evaporation in vacuo, the 
residue was crystallised from EtOH. Yield $=18 \%$ $(15 \mathrm{mg}) ; \mathrm{mp}=178-181{ }^{\circ} \mathrm{C}($ EtoH $) .{ }^{1} \mathrm{H}-\mathrm{NMR}(400 \mathrm{MHz}$, $\left.\mathrm{CDCl}_{3}\right) \delta 3.20\left(\mathrm{~s}, 3 \mathrm{H}, \mathrm{CH}_{3}\right), 7.10(\mathrm{~d}, 1 \mathrm{H}$, pyridopyrimidine $\mathrm{H} 9, J=6.8 \mathrm{~Hz}$ ), 7.70 (d, 1H, pyridopyrimidine $\mathrm{H} 7, J=8.8 \mathrm{~Hz}), 7.85(\mathrm{t}, 1 \mathrm{H}$, pyridopyrimidine $\mathrm{H} 8$, $J=7.6 \mathrm{~Hz}$ ), 9.15 (s, 1H, pyridopyrimidine $\mathrm{H} 2$ ), 12.65 (exch br s, $1 \mathrm{H}, \mathrm{COOH}$ ). Anal. Calcd. for $\mathrm{C}_{10} \mathrm{H}_{8} \mathrm{~N}_{2} \mathrm{O}_{3}$ : C 58.82, H 3.95, N 13.72. Found: C 58.69, H 3.96, N 13.75.

General procedure for the synthesis of pyridopyrimidine carboxamides 3a-f Compounds 3a-f were obtained starting from intermediate $\mathbf{1 7}$ following the same procedure described for 2c-i. After $40 \mathrm{~min}$ of stirring at room temperature, the solvent was removed, and the residue was purified by flash column chromatography using $\mathrm{CHCl}_{3} / \mathrm{MeOH}$ 9:1 (for 3a), $\mathrm{EtOH} / \mathrm{CHCl}_{3}$ /ethyl ether/petroleum ether/33\% $\mathrm{NH}_{4} \mathrm{OH}$ 4.5:18:18:45:0.25 (for 3b,d,e), toluene/ethyl acetate 1:2 (for 3c) or cyclohexane/ethyl acetate 1:1 (for $\mathbf{3 f}$ ) as eluents.

- 6-Methyl-4-oxo-N-methyl-4H-pyrido[1,2-a]pyrimidine3-carboxamide (3a)

Yield $=65 \%(34 \mathrm{mg}) ; \mathrm{mp}=201-203{ }^{\circ} \mathrm{C}(\mathrm{EtOH}) .{ }^{1} \mathrm{H}-\mathrm{NMR}$ (400 MHz, $\left.\mathrm{CDCl}_{3}\right) \delta 3.05$ (s, 3H, NH-CH ), 3.10 (s, 3H, $\left.\mathrm{CH}_{3}\right), 6.85-6.95(\mathrm{~m}, 1 \mathrm{H}$, pyridopyrimidine $\mathrm{H} 9), 7.55-7.70$ (m, 2H, pyridopyrimidine $\mathrm{H} 7$ and $\mathrm{H} 8$ ), 8.80 (exch br s, 1H, $\mathrm{NH}), 9.15$ (s, 1H, pyridopyrimidine $\mathrm{H} 2$ ). Anal. Calcd. for $\mathrm{C}_{11} \mathrm{H}_{11} \mathrm{~N}_{3} \mathrm{O}_{2}$ : C 60.82, H 5.10, N 19.34. Found: C 60.65, H 5.12, N 19.39. [25]

- 6-Methyl-4-oxo-N-phenyl-4H-pyrido[1,2-a]pyrimidine3-carboxamide (3b)

Yield $=45 \%(30 \mathrm{mg}) ; \mathrm{mp}=179-181{ }^{\circ} \mathrm{C}\left(\mathrm{Et}_{2} \mathrm{O}\right) .{ }^{1} \mathrm{H}-\mathrm{NMR}$ (400 MHz, $\mathrm{CDCl}_{3}$ ) $\delta 3.20$ (s, 3H, $\mathrm{CH}_{3}$ ), 7.00-7.05 (m, 1H, pyridopyrimidine $\mathrm{H} 9), 7.15$ (t, 1H, Ar, J=8.4 Hz), 7.35 (t, $2 \mathrm{H}, \mathrm{Ar}, J=7.8 \mathrm{~Hz}), 7.70-7.80(\mathrm{~m}, 4 \mathrm{H}, 2 \mathrm{H} \mathrm{Ar}+$ pyridopyrimidine $\mathrm{H} 7$ and $\mathrm{H} 8$ ), 9.25 (s, 1H, pyridopyrimidine $\mathrm{H} 2$ ), 11.00 (exch br s, $1 \mathrm{H}, \mathrm{NH}$ ). Anal. Calcd. for $\mathrm{C}_{16} \mathrm{H}_{13} \mathrm{~N}_{3} \mathrm{O}_{2}$ : C 68.81, H 4.69, N 15.05. Found: C 68.62, H 4.71, N 15.08. [25]

- 6-Methyl-N-(naphthalen-2-yl)-4-oxo-4H-pyrido[1,2-a] pyrimidine-3-carboxamide (3c)

Yield $=21 \%(16 \mathrm{mg}) ; \mathrm{mp}=176-179{ }^{\circ} \mathrm{C}(\mathrm{EtOH}) .{ }^{1} \mathrm{H}-\mathrm{NMR}$ (400 MHz, $\mathrm{CDCl}_{3}$ ) $\delta 3.30\left(\mathrm{~s}, 3 \mathrm{H}, \mathrm{CH}_{3}\right), 7.05-7.10(\mathrm{~m}, 1 \mathrm{H}$, pyridopyrimidine $\mathrm{H} 9), 7.55$ (t, 2H, Ar, J=7.6 Hz), 7.60 (t, $1 \mathrm{H}$, pyridopyrimidine $\mathrm{H} 8, J=8.4 \mathrm{~Hz}), 7.70(\mathrm{~d}, 1 \mathrm{H}$, pyridopyrimidine $\mathrm{H} 7, J=8.0 \mathrm{~Hz}$ ), 7.75-7.85 (m, 2H, Ar), 7.90 (d, 1H, Ar, $J=7.8 \mathrm{~Hz}$ ), 8.20 (d, 1H, Ar, $J=7.8 \mathrm{~Hz}), 8.50$ (d, 1H, Ar, $J=7.6 \mathrm{~Hz}$ ), 9.35 (s, 1H, pyridopyrimidine H2), 11.60 (exch br s, $1 \mathrm{H}, \mathrm{NH})$. Anal. Calcd. for $\mathrm{C}_{20} \mathrm{H}_{15} \mathrm{~N}_{3} \mathrm{O}_{2}$ : C 72.94, H 4.59, $\mathrm{N}$ 12.76. Found: C 72.75, H 4.59, N 12.73.

- N-Benzhydryl-6-methyl-4-oxo-4H-pyrido[1,2-a]pyrimidine3-carboxamide $(\mathbf{3 d})$

Yield $=15 \%(13 \mathrm{mg}) ; \mathrm{mp}=160-164{ }^{\circ} \mathrm{C}(\mathrm{EtOH}) .{ }^{1} \mathrm{H}-\mathrm{NMR}$ $\left(400 \mathrm{MHz}, \mathrm{CDCl}_{3}\right) \delta 3.15\left(\mathrm{~s}, 3 \mathrm{H}, \mathrm{CH}_{3}\right), 6.50(\mathrm{~d}, 1 \mathrm{H}, \mathrm{NH}-\mathrm{CH}$, $J=8.0 \mathrm{~Hz}), 6.90(\mathrm{~d}, 1 \mathrm{H}$, pyridopyrimidine $\mathrm{H} 9, J=6.4 \mathrm{~Hz}$ ),
7.25-7.30 (m, 2H, Ar), 7.30-7.40 (m, 8H, Ar), $7.60(\mathrm{~d}, 1 \mathrm{H}$, pyridopyrimidine $\mathrm{H} 7, J=8.0 \mathrm{~Hz}), 7.70(\mathrm{t}, 1 \mathrm{H}$, pyridopyrimidine $\mathrm{H} 8, J=7.2 \mathrm{~Hz}$ ), 9.20 (s, $1 \mathrm{H}$, pyridopyrimidine $\mathrm{H} 2$ ), 9.80 (d, $1 \mathrm{H}, \mathrm{NH}$ ). Anal. Calcd. for $\mathrm{C}_{23} \mathrm{H}_{19} \mathrm{~N}_{3} \mathrm{O}_{2}$ : C 74.78, $\mathrm{H}$ 5.18, N 11.37. Found: C 74.61, H 5.16, N 11.39.

- N,N-Dibenzyl-6-methyl-4-oxo-4H-pyrido[1,2-a]pyrimidine3-carboxamide (3e)

Yield $=15 \%$ (14 mg); $\mathrm{mp}=260-263^{\circ} \mathrm{C}$ (Petroleum ether). ${ }^{1} \mathrm{H}-\mathrm{NMR}\left(400 \mathrm{MHz}, \mathrm{CDCl}_{3}\right) \delta 3.10$ (s, 3H, $\mathrm{CH}_{3}$ ), 4.50 (s, 2H, $\mathrm{CH}_{2} \mathrm{Ph}$ ), 4.75 (s, $\left.2 \mathrm{H}, \mathrm{CH}_{2} \mathrm{-Ph}\right), 6.80$ (d, $1 \mathrm{H}$, pyridopyrimidine H9, $J=6.4 \mathrm{~Hz}$ ), 7.20 (d, 2H, Ar, $J=8.0 \mathrm{~Hz}$ ), 7.30-7.40 (m, $8 \mathrm{H}, \mathrm{Ar}), 7.50(\mathrm{~d}, 1 \mathrm{H}$, pyridopyrimidine $\mathrm{H} 7, J=7.8 \mathrm{~Hz})$, $7.55(\mathrm{t}, 1 \mathrm{H}$, pyridopyrimidine $\mathrm{H} 8, J=7.8 \mathrm{~Hz}), 8.35(\mathrm{~s}, 1 \mathrm{H}$, pyridopyrimidine $\mathrm{H} 2$ ). Anal. Calcd. for $\mathrm{C}_{24} \mathrm{H}_{21} \mathrm{~N}_{3} \mathrm{O}_{2}$ : C 75.18, H 5.52 N 10.96. Found: C 75.36, H 5.50, N 10.98.

- 6-Methyl-4-oxo-N,N-diphenyl-4H-pyrido[1,2-a]pyrimidine3-carboxamide (3f)

Yield $=40 \%(34 \mathrm{mg}) ; \mathrm{mp}=121-124{ }^{\circ} \mathrm{C}(\mathrm{EtOH}) .{ }^{1} \mathrm{H}-$ NMR (400 MHz, $\left.\mathrm{CDCl}_{3}\right) \delta 2.45\left(\mathrm{~s}, 3 \mathrm{H}, \mathrm{CH}_{3}\right), 6.70(\mathrm{~d}$, $1 \mathrm{H}$, pyridopyrimidine $\mathrm{H} 9, J=6.0 \mathrm{~Hz}), 6.95(\mathrm{t}, 2 \mathrm{H}, \mathrm{Ar}$, $J=7.2 \mathrm{~Hz}), 7.10(\mathrm{~d}, 4 \mathrm{H}, \mathrm{Ar}, J=8.0 \mathrm{~Hz}), 7.15-7.20(\mathrm{~m}$, $4 \mathrm{H}, \mathrm{Ar}), 7.40(\mathrm{~d}, 1 \mathrm{H}$, pyridopyrimidine $\mathrm{H} 7, J=9.2 \mathrm{~Hz})$, $7.50(\mathrm{t}, 1 \mathrm{H}$, pyridopyrimidine $\mathrm{H} 8, J=7.8 \mathrm{~Hz}), 8.55(\mathrm{~s}, 1 \mathrm{H}$, pyridopyrimidine $\mathrm{H} 2$ ). Anal. Calcd. for $\mathrm{C}_{22} \mathrm{H}_{17} \mathrm{~N}_{3} \mathrm{O}_{2}$ : C 74.35, H 4.82, N 11.82. Found: C 74.26, H 4.84, N 11.87.

\section{Molecular modelling}

The pairwise similarity was calculated by using ECFP4/FCFP4 circular fingerprints using Forge 10.6.0 Revision: 36,004 (Cresset Biomolecular Discovery Ltd.). Each ligand was then subjected to an optimisation process with the additions of hydrogens using YASARA (version 14.7.17, YASARA Biosciences). Using the same software, each ligand was subjected to an energy minimisation process to obtain 3D structures ready for molecular docking saved as a PDB file. Once obtained the 3D structures for all the compounds, the geometry was also optimised at semi-empirical level using the PM3 semi-empirical Hamiltonian as implemented in MOPAC 2016 package. A protein database, consisting of 31 crystallised proteins, was built through Protein Data Bank (www.rcsb.org) and then using YASARA the proteins was prepared by removing water and ions, and other co-crystallised molecules. Moreover, the protein structure was also optimised by the addition of hydrogens. A simulation cell was created in the binding pockets of the 31 selected proteins, defined as $2 \AA$ cube around the 3D ligand. The ligand was then removed, ready for the molecular docking process as a YASARA scene file. To obtain binding energies, molecular docking calculations were done using King's Virtual Desktop client running eight cores $2.59 \mathrm{Ghz}$ Intel Xenon processor and utilising the Autodock Vina software package applied in YASARA (i.e. dock_run.mcr). The highest docking calculation for each ligand was selected and imputed in a matrix. 


\section{Drug score and ADME assessment}

The current set of compounds was analysed with the PBPK modelling software platform PK-Sim/MoBi (Bayer Technology Services, Leverkusen, Germany) version 9. Physicochemical properties of the compounds and their unbound fractions are presented in Table S8. A uniform $20 \mathrm{mg}$ intravenous (I/V) and oral administration protocol applied to the European population (European ICRP 2002/Generic model PK-Sim-MoBi) were adopted for the compound dataset, due to the unavailability of data on minimum effective concentration, minimum toxic concentration and therapeutic window. European ICRP 2002 is a generic model structure, having the physiological database of predefined European population and its demographic features as reported in Table S10.

\section{Results and discussion}

\section{Heterocyclic small-molecule design}

To generate new series of molecular diverse compounds, we have used a two-step computing-assisted molecular design.
As showed in Fig. 1, the design focused on the search for bioisosteric-replacements/scaffold-hopping [26, 27] of the quinolinone scaffold. Quinolines and quinolinones are widely explored scaffolds in medicinal chemistry. The increase of their molecular diversity would result in expanding the array of biological targets and drug discovery applications for this chemotype [28, 29]. Our bioisosteric replacement analysis led to the selection of three nitrogen-containing heterocyclic frameworks, i.e. 3,7-dihydropyrimido[4,5-d]pyridazine4,8-dione, 1,8-naphthyridin-4(1H)-one and 4H-pyrido[1,2-a] pyrimidin-4-one, showing features of both synthetic accessibility and molecular diversity. In the second step of the design, a ligand growing approach was chosen to enlarge the library of compounds and reach optimum physico-chemical properties, i.e. molecular weight, cLogP, cLogS, H-bond-acceptors, $\mathrm{H}$-bond-donors, total surface area, relative polar surface area, shape index and flexibility [30, 31]. The results of the ligand growing experiments led to define 43 analogues reported in Tables 1, 2, and 3. To assess the molecular diversity within the compounds, the pairwise similarity was calculated by using ECFP4/FCFP4 circular fingerprints [32, 33]. The results of the calculated physico-chemical properties and the fingerprints analyses are reported in Figs. 2 and 3 and Tables S2 and S3

Table 1 Structures of the heterocyclic small-molecules with pyrimido[4,5-d]pyridazine-4,8-dione scaffold

\begin{tabular}{|c|c|c|c|c|}
\hline Comp. & $\mathbf{R}$ & $\mathbf{R}_{1}$ & $\mathbf{R}_{2}$ & $\mathbf{R}_{3}$ \\
\hline 1a & $\mathrm{Ph}$ & $\mathrm{C}_{2} \mathrm{H}_{5}$ & $\mathrm{C}_{2} \mathrm{H}_{5}$ & $\mathrm{H}$ \\
\hline $1 \mathrm{~b}$ & $\mathrm{Ph}$ & $\mathrm{CH}_{2}-\left(4-\mathrm{NO}_{2}\right)-\mathrm{Ph}$ & $\mathrm{H}$ & $\mathrm{H}$ \\
\hline 1c & $\mathrm{Ph}$ & $\mathrm{CH}_{2}-\left(4-\mathrm{COOC}_{2} \mathrm{H}_{5}\right)-\mathrm{Ph}$ & $\mathrm{H}$ & $\mathrm{H}$ \\
\hline 1d & $\mathrm{Ph}$ & $\mathrm{CH}_{2}-\left(4-\mathrm{CH}_{2} \mathrm{OCOCH}_{3}\right)-\mathrm{Ph}$ & $\mathrm{H}$ & $\mathrm{H}$ \\
\hline $1 \mathrm{e}$ & 3-F-Ph & $\mathrm{C}_{2} \mathrm{H}_{5}$ & $\mathrm{H}$ & $\mathrm{H}$ \\
\hline 1f & 3-Cl-Ph & $\mathrm{CH}_{2} \mathrm{cC}_{3} \mathrm{H}_{5}$ & $\mathrm{H}$ & $\mathrm{H}$ \\
\hline $1 \mathrm{~g}$ & 3-Cl-Ph & $\mathrm{CH}_{2} \mathrm{cC}_{6} \mathrm{H}_{11}$ & $\mathrm{H}$ & $\mathrm{H}$ \\
\hline $1 \mathrm{~h}$ & 3-Cl-Ph & $\mathrm{cC}_{6} \mathrm{H}_{11}$ & $\mathrm{H}$ & $\mathrm{H}$ \\
\hline $1 \mathbf{i}$ & $3-\mathrm{CH}_{3}-\mathrm{Ph}$ & $\mathrm{CH}_{2} \mathrm{CC}_{6} \mathrm{H}_{11}$ & $\mathrm{H}$ & $\mathrm{H}$ \\
\hline $\mathbf{1 j}$ & pyridin-3-yl & $\mathrm{CH}_{2} \mathrm{CC}_{6} \mathrm{H}_{11}$ & $\mathrm{H}$ & $\mathrm{H}$ \\
\hline $1 \mathbf{k}$ & $\mathrm{Ph}$ & $\mathrm{C}_{2} \mathrm{H}_{5}$ & $\mathrm{H}$ & $\mathrm{CH}_{3}$ \\
\hline 11 & $\mathrm{Ph}$ & $\mathrm{C}_{2} \mathrm{H}_{5}$ & $\mathrm{H}$ & $\mathrm{CH}_{2} \mathrm{Ph}$ \\
\hline $1 \mathrm{~m}$ & $\mathrm{Ph}$ & $\mathrm{CH}_{2}-(4-\mathrm{COOH})-\mathrm{Ph}$ & $\mathrm{H}$ & $\mathrm{H}$ \\
\hline 1n & $\mathrm{Ph}$ & $\mathrm{CH}_{2}-\left(4-\mathrm{CH}_{2} \mathrm{OH}\right)-\mathrm{Ph}$ & $\mathrm{H}$ & $\mathrm{H}$ \\
\hline 10 & $\mathrm{Ph}$ & $\mathrm{CH}_{2} \mathrm{CC}_{6} \mathrm{H}_{11}$ & $\mathrm{H}$ & $\mathrm{H}$ \\
\hline $1 p$ & $\mathrm{Ph}$ & $\mathrm{CH}_{2} \mathrm{Ph}$ & $\mathrm{H}$ & $\mathrm{H}$ \\
\hline $1 q$ & $\mathrm{Ph}$ & $\mathrm{CH}_{2} \mathrm{cC}_{3} \mathrm{H}_{5}$ & $\mathrm{H}$ & $\mathrm{H}$ \\
\hline $1 \mathbf{r}$ & pyridin-3-yl & $\mathrm{CH}_{2}-(3-\mathrm{Cl})-\mathrm{Ph}$ & $\mathrm{H}$ & $\mathrm{H}$ \\
\hline $1 \mathrm{~s}$ & $\mathrm{Ph}$ & $\mathrm{CH}_{2} \mathrm{cC}_{3} \mathrm{H}_{5}$ & $\mathrm{C}_{2} \mathrm{H}_{5}$ & $\mathrm{H}$ \\
\hline $1 t$ & $\mathrm{Ph}$ & $\mathrm{CH}_{2} \mathrm{CC}_{6} \mathrm{H}_{11}$ & $\mathrm{H}$ & $\mathrm{C}_{2} \mathrm{H}_{5}$ \\
\hline $1 \mathrm{u}$ & $\mathrm{Ph}$ & $\mathrm{CH}_{2} \mathrm{cC}_{3} \mathrm{H}_{5}$ & $\mathrm{H}$ & $\mathrm{C}_{2} \mathrm{H}_{5}$ \\
\hline
\end{tabular}


Table 2 Structures of the heterocyclic small-molecules with 1,8-naphtyridin-4-one scaffold

\begin{tabular}{|c|c|c|c|}
\hline Comp. & $\mathbf{R}_{1}$ & $\mathbf{R}$ & $\mathbf{R}_{\mathbf{2}}$ \\
\hline $2 \mathbf{a}$ & $\mathrm{CH}_{2}-(4-\mathrm{Cl})-\mathrm{Ph}$ & $\mathrm{COOCH}_{2} \mathrm{CH}_{3}$ & $\mathrm{CH}_{3}$ \\
\hline $2 \mathbf{b}$ & $3-\mathrm{NO}_{2}-\mathrm{Ph}$ & $\mathrm{COOCH}_{2} \mathrm{CH}_{3}$ & $\mathrm{CH}_{3}$ \\
\hline 2c & $\mathrm{CH}_{2} \mathrm{CH}_{3}$ & CONH- pyridin-3-yl & $\mathrm{CH}_{3}$ \\
\hline 2d & $\mathrm{CH}_{2} \mathrm{CH}_{3}$ & $\mathrm{CONHNHCOCH}_{3}$ & $\mathrm{CH}_{3}$ \\
\hline $2 \mathrm{e}$ & $\mathrm{CH}_{2} \mathrm{CH}_{3}$ & $\mathrm{CON}\left(\mathrm{CH}_{3}\right) \mathrm{Ph}$ & $\mathrm{CH}_{3}$ \\
\hline $2 f$ & $\mathrm{CH}_{2} \mathrm{CH}_{3}$ & CONH-naphtalen-2-yl & $\mathrm{CH}_{3}$ \\
\hline $2 \mathrm{~g}$ & $\mathrm{CH}_{2}-(4-\mathrm{Cl})-\mathrm{Ph}$ & $\mathrm{CONHCH}_{3}$ & $\mathrm{CH}_{3}$ \\
\hline $2 \mathrm{~h}$ & $3 \mathrm{~F}-\mathrm{Ph}$ & $\mathrm{CONHCH}_{3}$ & $\mathrm{CH}_{3}$ \\
\hline $2 \mathbf{i}$ & $3 \mathrm{~F}-\mathrm{Ph}$ & $\mathrm{CONHNHCOCH}_{3}$ & $\mathrm{CH}_{3}$ \\
\hline $\mathbf{2} \mathbf{j}$ & $\mathrm{CH}_{2} \mathrm{CH}_{3}$ & CONH-(3,5-dichloropyridin)-4-yl & $\mathrm{CH}_{3}$ \\
\hline $2 \mathbf{k}$ & $\mathrm{CH}_{2}-(4-\mathrm{F})-\mathrm{Ph}$ & CONH-(3,5-dichloropyridin)-4-yl & $\mathrm{CH}_{3}$ \\
\hline 21 & $\mathrm{CH}_{2} \mathrm{CH}_{3}$ & 5-methyl-1,3,4-oxadiazol-2-yl & $\mathrm{CH}_{3}$ \\
\hline $2 \mathrm{~m}$ & $\mathrm{CH}_{2} \mathrm{CH}_{3}$ & $\mathrm{COOCH}_{2} \mathrm{CH}_{3}$ & $3-\mathrm{NO}_{2}-\mathrm{Ph}$ \\
\hline 2n & $\mathrm{CH}_{2} \mathrm{CH}_{3}$ & CONHPh & $\mathrm{OCH}_{3}$ \\
\hline 20 & $\mathrm{CH}_{2} \mathrm{CH}_{3}$ & CONHPh & pyrrolidin-1-yl \\
\hline $2 \mathbf{p}$ & $\mathrm{CH}_{2} \mathrm{CH}_{3}$ & $\mathrm{CON}\left(\mathrm{CH}_{3}\right) \mathrm{Ph}$ & pyrrolidin-1-yl \\
\hline
\end{tabular}

(see Supporting Information). ECFP4/FCFP4 circular fingerprints showed that the new set of compounds covers a wide range of molecular features (in line with the structural diversity investigated in this study), which can be summarised as follows: molecular weight, from 217 to 457 ; cLogP, from -0.83 to 3.81; cLogS, from -6.56 to-1.26; H-acceptors, between 5 and 9; H-donors, between 0 and 2; total polar surface, from 167 to 321 ; relative polar surface area, from 0.15 to 0.35 ; shape index, from 0.43 to 0.58 ; flexibility, from 0.28 to 0.53 .

\section{Chemistry}

The synthetic procedures carried out to obtain the 43 target compounds of type 1-3 are reported in Schemes 1, 2, 3 and
4. The structures were confirmed on the basis of analytical and spectral data. Scheme 1 shows the synthetic pathway affording the final compounds 1a-n containing the new bicyclic scaffold pyrimido[4,5-d]pyridazine-4,8-dione. Intermediates 5a-e (5a [34] and 5b,5d [22]) were obtained starting from isoxazole $4 \mathbf{a}-\mathbf{e}$ (where $4 \mathrm{c}$ and $4 \mathrm{e}$ are commercially available, while compounds $\mathbf{4 a}, \mathbf{4 b}$ and $\mathbf{4 d}$ were synthesised by adopting previously reported protocols) [22, 35] using hydrazine hydrate and polyphosphoric acid (PPA) in ethanol. The 4-amino-5-amido derivatives 6 a-e were obtained by reacting analogues of type 5 with $33 \% \mathrm{NH}_{4} \mathrm{OH}$ at $60{ }^{\circ} \mathrm{C}$. The alkylation of 6a-e with suitable alkyl(aryl) halides in standard conditions afforded the intermediates $\mathbf{7 a - d}$ and $\mathbf{7 f -}$ $\mathbf{k}$ (where 7a,f were previously reported) [22]. Differently,
Table 3 Structures of the heterocyclic small-molecules with pyrido[1,2-a]pyrimidine scaffold

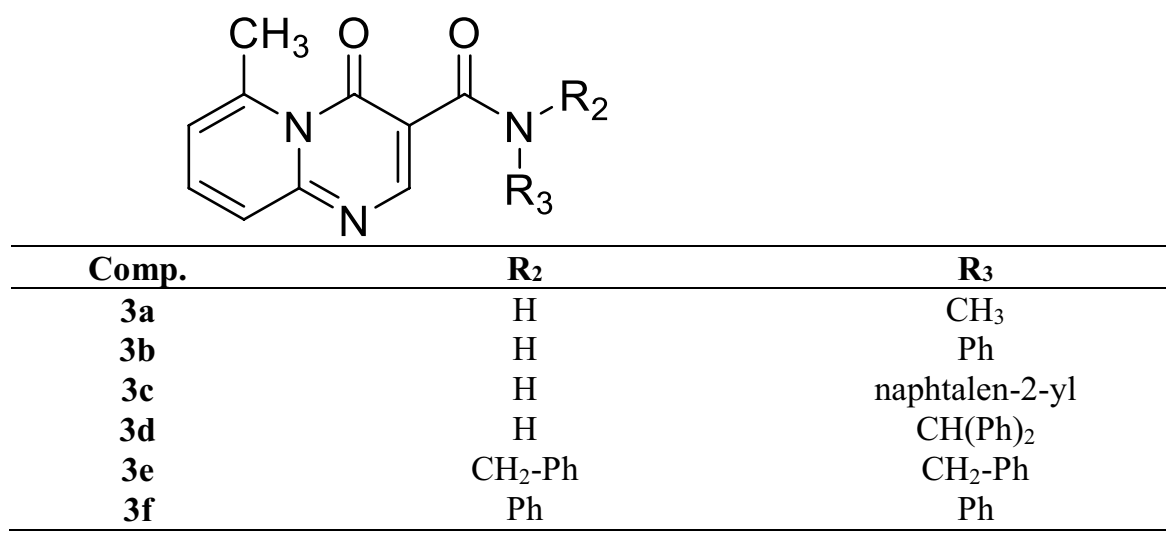




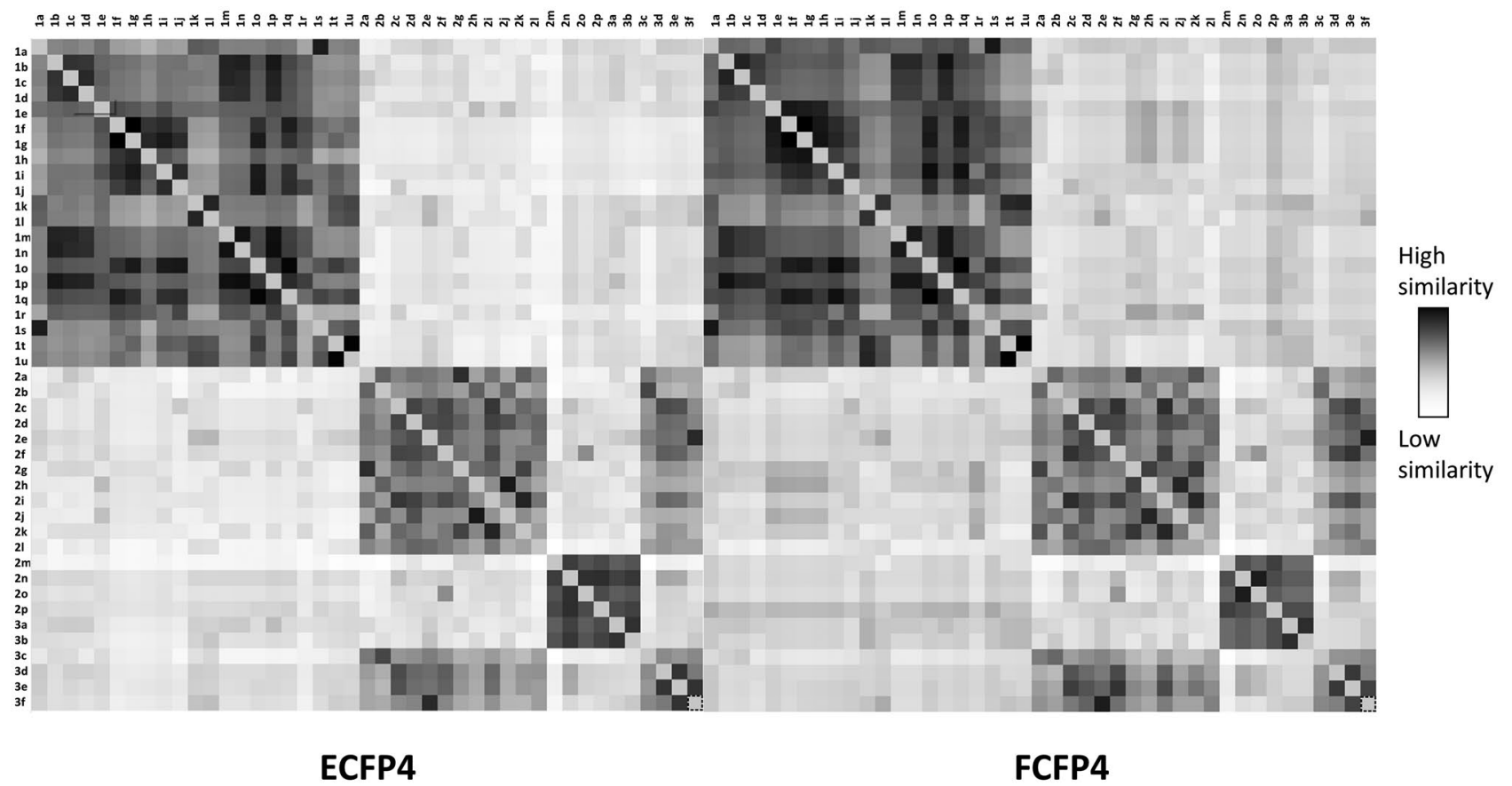

Fig. 2 ECFP4 and FCFP4 fingerprint similarity matrix

compound $7 \mathbf{e}$ was obtained from $\mathbf{7 d}$ by treatment with acetyl chloride in anhydrous $\mathrm{CH}_{2} \mathrm{Cl}_{2}$ at room temperature. Lastly, compounds 1a-j and $\mathbf{8}$ were obtained by the cyclisation of intermediates of type 7 with triethylorthoformate or triethylorthopropionate in $\mathrm{H}_{2} \mathrm{SO}_{4}$. For the synthesis of $\mathbf{1 ~ k , l , ~ c o m - ~}$ pound $\mathbf{8}$ was alkylated with the appropriate alkyl halide in standard conditions, while compounds $\mathbf{1} \mathbf{~ m , n}$ were obtained starting from 1c and 1d through hydrolysis with $\mathrm{NaOH}$ in $\mathrm{EtOH}$ at room temperature.

Scheme 2 reports the synthesis of the final compounds 1o-u also showing the pyrimido[4,5-d]pyridazine-4,8-dione scaffold. The pyridazinones 9a [22] and $\mathbf{9 b}$ were obtained starting from $\mathbf{5 a}$ and $\mathbf{5 e}$, by reductive cleavage with piperidine in anhydrous $\mathrm{CH}_{3} \mathrm{OH}$ at $60{ }^{\circ} \mathrm{C}$. After alkylation in standard conditions with the appropriate alkyl halide to give 10a-d, the 4-ammino-5-carboxyl derivatives 11a-d were obtained through hydrolysis with $6 \mathrm{~N} \mathrm{NaOH}$ in EtOH at $60-70{ }^{\circ} \mathrm{C}$. 11a-d were then converted by treatment with $\mathrm{SOCl}_{2}, \mathrm{Et}_{3} \mathrm{~N}$ and $33 \% \mathrm{NH}_{3}$ into the corresponding amide derivatives 12a-d which, in turn, were cyclised to final compounds 10-s with triethylorthoformate or triethylorthopropionate, by adopting the same procedure described in Scheme 1. Lastly, the alkylation of $\mathbf{1 0}$ and $\mathbf{1 q}$ with ethyl bromide afforded the final compounds $\mathbf{1 t}, \mathbf{u}$.

The naphtyridone scaffold 15 (Scheme 3) was synthesised from 2-amino-6-methylpyridine 13 and diethyl2-(ethoxymethylene)malonate (EMME), through a simultaneous and thermally induced ring closure of the diethyl-(6-methyl2-pyridylaminomethylene)malonate intermediate $\mathbf{1 4}[36,37]$.
Compound 15 was treated with the suitable alkyl/aryl halide in standard alkylation conditions (for 18a,b, 2a) [38], or coupled with the appropriate R-phenylboronic acid in the presence of $\mathrm{Cu}(\mathrm{OAc})_{2}$ to obtain compounds $\mathbf{1 8 c}, \mathbf{d}$ and $\mathbf{2 b}$. Subsequently, the alkaline hydrolysis of the ester group afforded the carboxylic acids 19a-e (19a [38]) which were firstly converted in the corresponding acid chlorides using $\mathrm{SOCl}_{2}$ at room temperature and then treated with the appropriate amine in dry tetrahydrofuran to give the final compounds $\mathbf{2 c}-\mathbf{k}$. The oxadiazole derivative $\mathbf{2} \mathbf{I}$ was synthesised from the acetyl carboxyhydrazide $\mathbf{2 d}$ using $\mathrm{POCl}_{3}$ on $\mathrm{SiO}_{2}$ as cyclodehydrating agent. During the synthesis of intermediate 15, a side-reaction following the Gould-Jacobs mechanism afforded the isomer 4H-pyrido[1,2-a]pyrimidine $\mathbf{1 6}$ [24]. The latter was used to synthesise the pyrido(1,2-a)pyrimidine analogues 3a-f through the following steps: (i) treatment with $\mathrm{HCl}$ in acetic acid at reflux (to obtain the corresponding carboxylic acid 17) and (ii) conversion into the final products 3a-f using the same procedure adopted for $\mathbf{2 c - k}$.

Scheme 4 reports the synthesis of the naphthyridone-based amide derivatives $\mathbf{2 n - p}$ and the 3-carbethoxy $\mathbf{2} \mathbf{~ m}$ bearing at position 7 a methoxy, a pyrrolidine or a $3-\mathrm{NO}_{2}$-phenyl substituent. Starting from intermediates of type 20 [23, 39, 40], the alkylation reaction with bromoethane e potassium carbonate in dry DMF afforded the derivatives 21a,b [23, 39]. Differently, the derivative $\mathbf{2} \mathbf{~ m}$ was obtained through Suzuki coupling performed on 20c and using 3-nitrophenylboronic acid, $2 \mathrm{M}$ sodium carbonate and tetrakis(triphenylphosphine)palladium $(0)$ in toluene. Alkaline hydrolysis of 21a,b generated the carboxylic acids 22a,b $[39,41]$ which, in turn, 

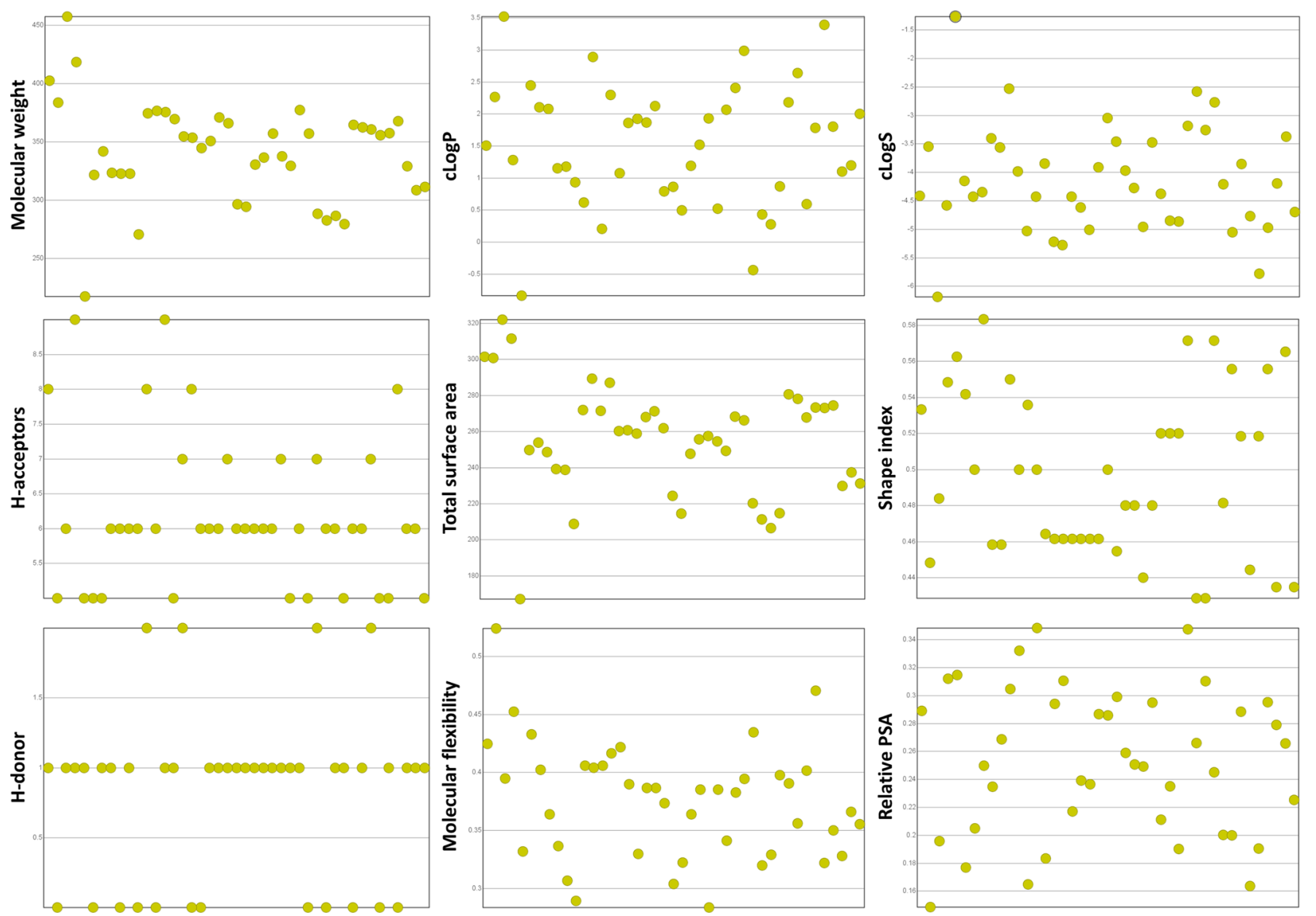

Fig. 3 Variability of physico-chemical properties within the library of 43 compounds

was converted into the final amides 2n-p through the acid chlorides intermediates, in usual conditions.

\section{Molecular modelling}

Adopting iVS methodologies, the library of compounds reported here (43 terms) was subsequently screened against a panel of 31 cellular targets, that are involved in the development, proliferation or progression of cancer in order to find potential hits for biomedical studies (Table S1, Supporting Information). The 31 targets were selected from a previously reported dataset, where a panel of several proteins involved in cancer was successfully validated for iVS investigations [17, 20, 21]. In this study, protein targets with diverse physiological functions were selected for the screening. Specifically, we have usedkinases with various roles (phospho-CDK2/ Cyclin A, B-Raf, PLK1, AKT, Pi3K, CK2, human anaplastic lymphoma kinase, PAK6, MDM2, MST3, C-SRC, MRCK, human ROS1 kinase, C-Abl kinase, PTK6, BRAF, ephrin A2 receptor protein kinase), grow factors (EGFR, C-Met, VEGFR2, FGFR1), transporters (FABP4), chaperones (HPS90), cellular enzymes (17-beta-hydroxysteroid dehydrogenase type1, PARP, matriptase, DNPH1, carbonic anhydrase IX), BCL6 (a transcription factor) and the human retinoid $\times$ receptor alpha. AutoDock Vina was used as the docking software [20,21]. The analysis of binding energies between the new heterocyclic ligands and targets was reported in Table S4 (see Supporting Information). Docking analysis of crystallised ligands, with an established binding mode, was carried out in order to obtain a minimum energy level which has been used as the cut-off for the assessment of binding energies of the new ligands. In particular, the binding efficiency was evaluated through the ratio between the binding energies of analysed ligands and reference ligands co-crystallised in the protein, by applying Eq. (1):

$\delta=\frac{\Delta G \text { compound }}{\Delta G \text { reference ligand }}$ 


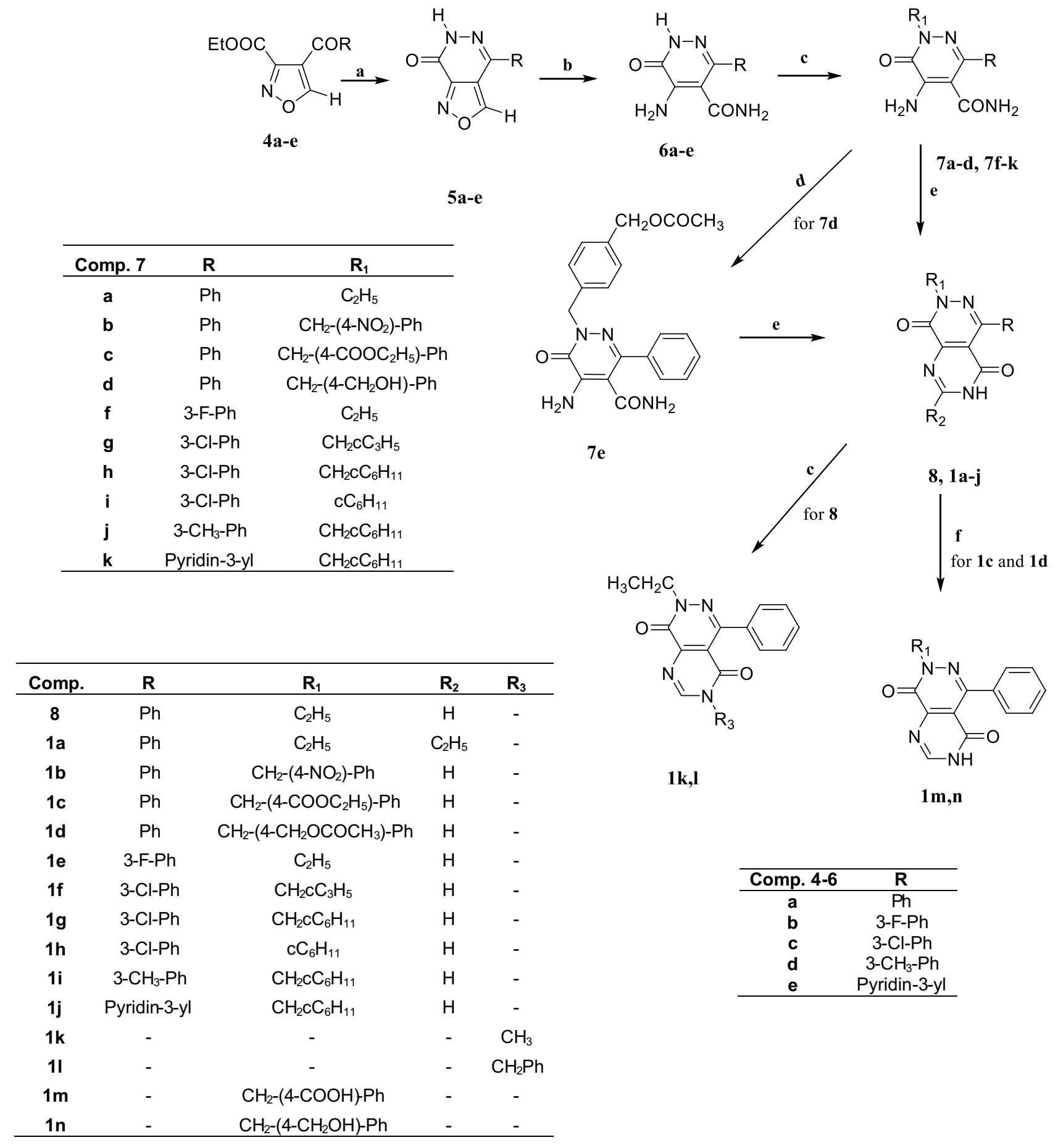

Scheme 1 Reagents and conditions: (a) $\mathrm{NH}_{2} \mathrm{NH}_{2} \cdot \mathrm{H}_{2} \mathrm{O}$, PPA, EtOH, 80-90 ${ }^{\circ} \mathrm{C}, 2-5 \mathrm{~h}$; (b) $33 \% \mathrm{NH}_{4} \mathrm{OH}, 60{ }^{\circ} \mathrm{C}, 1-2 \mathrm{~h}$; (c) suitable alkyl(aryl) halide, $\mathrm{K}_{2} \mathrm{CO}_{3}$, DMF, $80{ }^{\circ} \mathrm{C}, 1-3 \mathrm{~h}$; (d) acetyl chloride, anhydrous $\mathrm{CH}_{2} \mathrm{Cl}_{2}$, rt, $3 \mathrm{~h}$; (e) triethylorthoformate (or triethylorthopropionate), $\mathrm{H}_{2} \mathrm{SO}_{4}$ conc, $130^{\circ} \mathrm{C}, 1-5 \mathrm{~h}$; (f) $6 \mathrm{~N} \mathrm{NaOH}, \mathrm{EtOH}, \mathrm{rt}, 1-2 \mathrm{~h}$

Equation (1) was used as an inclusion criterion to assess which are the compounds worth further analysis. Only the analogues achieving a $\delta \geq 1$ were processed through a normalisation procedure. This approach allows to select and examine only the proteins showing the highest affinity for the ligands. The matrix containing $\delta$ calculations 
Scheme 2 Reagents and conditions: (a) anhydrous $\mathrm{MeOH}$, piperidine, $65^{\circ} \mathrm{C}, 1.5 \mathrm{~h}$; (b) suitable alkyl halide, $\mathrm{K}_{2} \mathrm{CO}_{3}$, DMF, $80{ }^{\circ} \mathrm{C}$, 30-90 $\mathrm{min}$; (c) $6 \mathrm{~N} \mathrm{NaOH}, \mathrm{EtOH}, 60-70{ }^{\circ} \mathrm{C}$, 1-2 h; (d) step1: $\mathrm{SOCl}_{2}, \mathrm{Et}_{3} \mathrm{~N}$, 60-70 ${ }^{\circ} \mathrm{C}, 1 \mathrm{~h}$; step 2: $33 \%$ $\mathrm{NH}_{4} \mathrm{OH}, 0^{\circ} \mathrm{C}, 30-60 \mathrm{~min}$; (e) triethylorthoformate (or triethylorthopropionate), $\mathrm{H}_{2} \mathrm{SO}_{4}$ conc., $130{ }^{\circ} \mathrm{C}, 2-4 \mathrm{~h}$; (f) $\mathrm{CH}_{3} \mathrm{CH}_{2} \mathrm{Br}$, $\mathrm{K}_{2} \mathrm{CO}_{3}$, dry DMF, $80{ }^{\circ} \mathrm{C}$, $30 \mathrm{~min}$
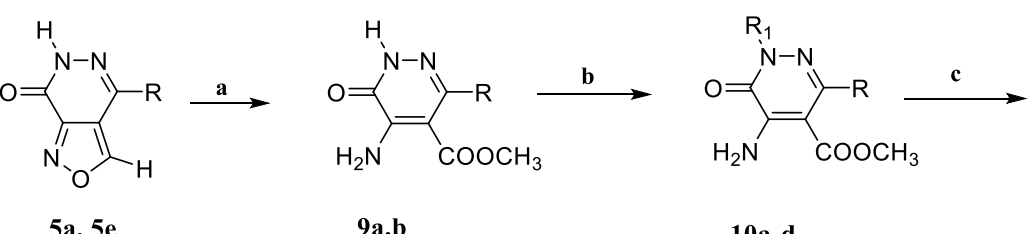<smiles>[R]c1nn([R])c(=O)c(N)c1C(=O)O</smiles>

10a-d

\begin{tabular}{cc}
\hline $\mathbf{9}$ & $\mathbf{R}$ \\
\hline $\mathbf{a}$ & $\mathrm{Ph}$ \\
$\mathbf{b}$ & Pyridin-3-yl \\
\hline
\end{tabular}
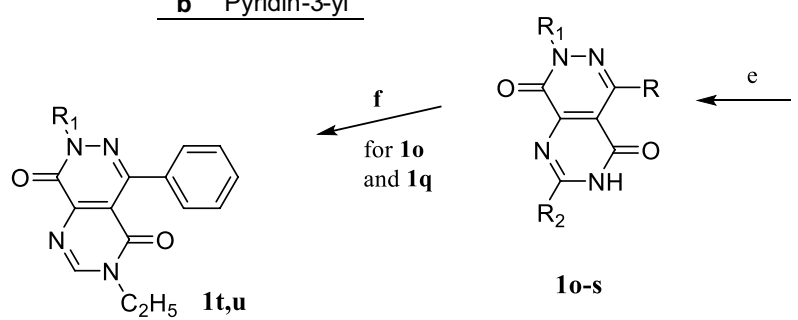<smiles>[R]c1nn([R])c(=O)c(N)c1C(N)=O</smiles>

10-s

\begin{tabular}{ccc}
\hline Comp. 10-12 & $\mathbf{R}$ & $\mathbf{R}_{\mathbf{1}}$ \\
\hline $\mathbf{a}$ & $\mathrm{Ph}$ & $\mathrm{CH}_{2} \mathrm{CC}_{6} \mathrm{H}_{11}$ \\
b & $\mathrm{Ph}$ & $\mathrm{CH}_{2}-\mathrm{Ph}$ \\
c & $\mathrm{Ph}$ & $\mathrm{CH}_{2} \mathrm{CC}_{3} \mathrm{H}_{5}$ \\
d & Pyridin-3-yl & $\mathrm{CH}_{2}-(3-\mathrm{Cl})-\mathrm{Ph}$ \\
\hline
\end{tabular}

(Table S5, Supporting Information) highlighted 17 proteins (PDB codes: 1XKK, 2FB8, 2RKU, 2XAB, 3L3L, 3LBZ, 3PE1, 3ZXZ, 4ASE, 4KS8, 4P5E, 4Q07, 4QMZ, 4U5J, 4UAL, 4YC8 and 5I9Y) where some specific compounds of the library express good to high affinity. In addition, we found higher affinity towards most of the ligands for the two proteins $2 \mathrm{RKU}$ and $4 \mathrm{Y} 5 \mathrm{~J}$, with $2 \mathrm{RKU}$ having higher binding energies than the co-crystallised ligand in all dockings and 4Y5J having only two results that were not higher than the co-crystallised ligand.

Similarly, several ligands have shown affinity for more than one cellular protein. Examining the $\delta$ calculation matrix, seven ligands have greater $\delta$ for 10 or more targets. These are the ligands $\mathbf{1 b}, \mathbf{1} \mathbf{g}, \mathbf{1} \mathbf{~ m}, \mathbf{2 f}, \mathbf{2 j}, \mathbf{2} \mathbf{k}, \mathbf{3 c}, \mathbf{3 d}$, which can be valid hits for multitarget pharmacological approach [42]. Moreover, four ligands (i.e. 1u, 2a, 2d and 2e) show selectivity towards one single protein, hence demonstrating possible candidates for targeted therapy. In contrast, the $\delta$ calculation matrix shows that only one ligand, 3a, did not have a $\delta \geq 1$ for any of the 31 proteins.

Normalisation of the binding energies values was conducted as proposed by Lauro et al. [20, 21] in order to overcome the lack of selectivity and occurrence of false positives, as well as to avoid systematic errors associated with the interaction of ligands and biological targets. Equation (2) was used to normalise the binding energy values in the matrix:

$\mathrm{V}=\mathrm{V} 0 /[(\mathrm{ML}+\mathrm{MR}) / 2]$

In this formula, $\mathrm{V}$ is the value associated with each compound and can be used to generate a ranking system between a protein and a ligand. V0 is the value of the binding energy (kcal/mol) from the molecular docking calculations, ML is the average binding energy $(\mathrm{kcal} / \mathrm{mol})$ of each ligand on different targets and MR is the average binding energy (kcal/ mol) associated with each target on various targets. Every single value in the matrix representing a single ligand versus a specific receptor was normalised taking simultaneously into account the specific averages contained in $\mathrm{V}$ values (Table S6, Supporting Information), which were also calculated for ligands co-crystallised in the protein in order to show significant interactions within the matrix.

The values obtained led to the identification of several compounds showing interaction with a number of proteins, highlighting 16 targets (PDB codes: 1XKK, 2FB8, 2RKU, 2XAB, 3L3L, 3PE1, 3ZXZ, 4ASE, 4KS8, 4P5E, 4Q07, $4 \mathrm{QMZ}, 4 \mathrm{UJ}$, 4UAL, 4YC8 and 5I9Y) from the 17 already selected with the Eq. (1). Specifically, these cellular proteins showed a higher trend of $\mathrm{V}$ values for the compound dataset, in comparison to the $\mathrm{V}$ values of the specific co-crystallised 
Scheme 3 Reagents and conditions: (a) EMME, toluene, $100{ }^{\circ} \mathrm{C}, 6 \mathrm{~h}$; (b) diphenyl ether, $270^{\circ} \mathrm{C}, 4 \mathrm{~h}$; (c) for $2 \mathrm{a}$ and 18a,b: alkyl halide, $\mathrm{K}_{2} \mathrm{CO}_{3}$, DMF, $60^{\circ} \mathrm{C}, 30-180 \mathrm{~min}$; for $18 \mathrm{c}, \mathrm{d}$ and $2 \mathrm{~b}$ : R-arylboronic acid, $\mathrm{Cu}(\mathrm{OAc})_{2}, \mathrm{Et}_{3} \mathrm{~N}, \mathrm{CH}_{2} \mathrm{Cl}_{2}$, $24 \mathrm{~h}, \mathrm{rt}$; (d) $6 \mathrm{~N} \mathrm{NaOH}, \mathrm{EtOH}$, $0.5-1 \mathrm{~h}, \mathrm{rt}$; (e) for $2 \mathrm{c}-\mathrm{i}$, step 1 : $\mathrm{SOCl}_{2}$ (excess), Et3N (catalytic), 2-3 h, rt; step 2: appropriate amine, anhydrous THF, $2-3 \mathrm{~h}, \mathrm{rt}$; for $2 \mathrm{j}, \mathrm{k}$, step 1: $\mathrm{SOCl}_{2}$ (excess), Et3N (catalytic),

2-3 h, rt; step 2: 3,5-dichloro4-pyridilamina, $\mathrm{NaH}$, anhydrous THF, 30 min, rt; (f) $\mathrm{POCl}_{3}$ / $\mathrm{SiO}_{2}$, toluene, $80^{\circ} \mathrm{C}, 5 \mathrm{~h} ;(\mathbf{g})$ $6 \mathrm{M} \mathrm{HCl}, \mathrm{AcOH}$, reflux, $3 \mathrm{~h}$<smiles>[R3]N([R])C(=O)c1cnc2cccc(C)n2c1=O</smiles><smiles>CCOC(=O)c1cnc2cccc(C)n2c1=O</smiles><smiles>CCOC(=O)C(/C=N\c1nc(C)ccc1OCC)C(=O)OCC</smiles>
13

14<smiles>[R7]n1cc(C(=O)OCC)c(=O)c2ccc(C)nc21</smiles>

2a,b, 18a-d

19a-e

\begin{tabular}{cc}
\hline Comp. & $\mathbf{R}_{\mathbf{1}}$ \\
\hline 18a,19a & $\mathrm{CH}_{2} \mathrm{CH}_{3}$ \\
19b & $\mathrm{CH}_{2}-(4-\mathrm{Cl})-\mathrm{Ph}$ \\
18b,19c & $\mathrm{CH}_{2}-(4-\mathrm{F})-\mathrm{Ph}$ \\
18c,19d & $\mathrm{Ph}$ \\
18d,19e & $3 \mathrm{~F}-\mathrm{Ph}$ \\
\hline
\end{tabular}

21

\begin{tabular}{ccc}
\hline $\mathbf{3}$ & $\mathbf{R}_{\mathbf{2}}$ & $\mathbf{R}_{\mathbf{3}}$ \\
\hline $\mathbf{a}$ & $\mathrm{H}$ & $\mathrm{CH}_{3}$ \\
$\mathbf{b}$ & $\mathrm{H}$ & $\mathrm{Ph}$ \\
$\mathbf{c}$ & $\mathrm{H}$ & naphtalen-2-yl \\
$\mathbf{d}$ & $\mathrm{H}$ & $\mathrm{CH}(\mathrm{Ph})_{2}$ \\
$\mathbf{e}$ & $\mathrm{CH}_{2}-\mathrm{Ph}$ & $\mathrm{CH}_{2}-\mathrm{Ph}$ \\
$\mathbf{f}$ & $\mathrm{Ph}$ & $\mathrm{Ph}$ \\
\hline
\end{tabular}

\begin{tabular}{ccc}
\hline $\mathbf{2}$ & $\mathbf{R}_{\mathbf{1}}$ & $\mathbf{R}$ \\
\hline $\mathbf{a}$ & $\mathrm{CH}_{2}-(4-\mathrm{Cl})-\mathrm{Ph}$ & - \\
$\mathbf{b}$ & $3-\mathrm{NO}_{2}-\mathrm{Ph}$ & - \\
$\mathbf{c}$ & $\mathrm{CH}_{2} \mathrm{CH}_{3}$ & $\mathrm{NH}-$ pyridin-3-yl \\
$\mathbf{d}$ & $\mathrm{CH}_{2} \mathrm{CH}_{3}$ & $\mathrm{NHNHCOCH}$ \\
$\mathbf{e}$ & $\mathrm{CH}_{2} \mathrm{CH}_{3}$ & $\mathrm{~N}\left(\mathrm{CH}_{3}\right) \mathrm{Ph}_{3}$ \\
$\mathbf{f}$ & $\mathrm{CH}_{2} \mathrm{CH}_{3}$ & $\mathrm{NH}-$ naphtyl-3-yl \\
$\mathbf{g}$ & $\mathrm{CH}_{2}-(4-\mathrm{Cl})-\mathrm{Ph}$ & $\mathrm{NHCH}$ \\
$\mathbf{h}$ & $3 \mathrm{~F}-\mathrm{Ph}$ & $\mathrm{NHCH}$ \\
$\mathbf{i}$ & $3 \mathrm{~F}-\mathrm{Ph}$ & $\mathrm{NHHCOCH}_{3}$ \\
$\mathbf{j}$ & $\mathrm{CH}_{2} \mathrm{CH}$ & $\mathrm{NH}-(3,5-$ dichloropyridin)-4-yl \\
$\mathbf{k}$ & $\mathrm{CH}_{2}-(4-\mathrm{F})-\mathrm{Ph}$ & $\mathrm{NH}-(3,5-$ dichloropyridin)-4-yl
\end{tabular}

inhibitor. $\mathrm{V}$ values against the selected targets are summarised in Table 4 and Fig. 4.

As mentioned earlier, all the proteins examined in this study are established targets with key roles in cancer pathogenesis. Moreover, 12 out of the 16 selected proteins which showed good affinity for the ligands are protein kinases. In this regard, the pyrimido[4,5-d]pyridazine-4,8-dione scaffold is a bi-cyclic structure containing a fused pyridazinone heterocycle [43, 44], which is a privileged structural motif for targeting the cellular proteins investigated in this study. For example, several previously reported inhibitors of VEGF-2, PDE4, c-met and EGFR inhibitors are based on the pyridazinone scaffold [45-47]. Pyridazine-based drugs such as olaparib and fuzuopali are also targeted to PARP for use in ovarian, breast, gastric and prostate solid tumours. Likewise, pyridine-containing molecules are inhibitors of B-Raf kinase and PLK 1 [48], as well as naphthyridine-based scaffolds, have been investigated for antiproliferative activity [49]. These literature evidences support our iVS study as an effective approach to identify hits from a database of ligands showing affinity for protein targets with relevance in cancer pathogenesis.

For selection and comparison of ligands which can be useful hits for biomedical studies, the molecules were 
Scheme 4 Reagents and conditions: (a) for 21a,b: $\mathrm{Br}-$ $\mathrm{CH}_{2} \mathrm{CH}_{3}, 20 \mathrm{a}$ or $20 \mathrm{~b}, \mathrm{~K}_{2} \mathrm{CO}_{3}$, DMF, $60{ }^{\circ} \mathrm{C}, 30-180 \mathrm{~min}$; for $2 \mathrm{~m}$ : 3-nitrophenylboronic acid, 20c, tetrakis, $2 \mathrm{M} \mathrm{Na}_{2} \mathrm{CO}_{3}$, ethanol, toluene, $60{ }^{\circ} \mathrm{C}, 2-3 \mathrm{~h}$; (b) $\mathrm{NaOH} 6 \mathrm{~N}$, EtOH, 0.5-1 h, rt; (c) step 1: $\mathrm{SOCl}_{2}$ (excess), $\mathrm{Et}_{3} \mathrm{~N}$ (catalytic), 2-3 h, rt; step 2: appropriate amine, anhydrous THF, 2-3 h, rt

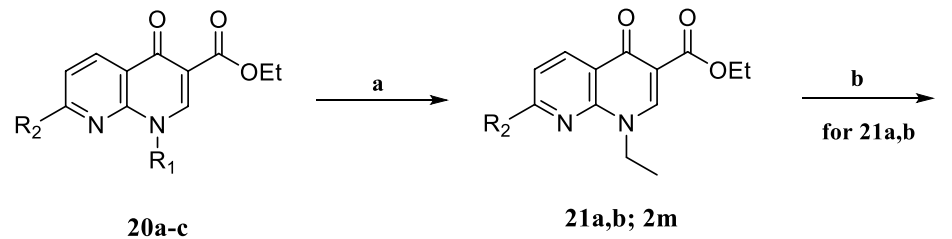<smiles>[R2]c1ccc2c(=O)c(C(=O)O)cn(CC)c2n1</smiles>

\begin{tabular}{ccc}
\hline $\mathbf{2 0}$ & $\mathbf{R}_{\mathbf{2}}$ & $\mathbf{R}_{\mathbf{1}}$ \\
\hline $\mathbf{a}$ & $\mathrm{OCH}_{3}$ & $\mathrm{H}$ \\
$\mathbf{b}$ & pyrrolidin-1-yl & $\mathrm{H}$ \\
$\mathbf{c}$ & $\mathrm{Cl}$ & $\mathrm{CH}_{2} \mathrm{CH}_{3}$ \\
\hline
\end{tabular}

c<smiles>[R]C(=O)c1cn(CC)c2nc([R])ccc2c1=O</smiles>

2n-p

\begin{tabular}{|c|c|c|}
\hline Comp. & $\mathbf{R}_{\mathbf{2}}$ & $\mathbf{R}$ \\
\hline $2 m$ & $3-\mathrm{NO}_{2}-\mathrm{Ph}$ & - \\
\hline $2 n$ & $\mathrm{OCH}_{3}$ & $\mathrm{NHPh}$ \\
\hline 20 & pyrrolidin-1-yl & NHPh \\
\hline $2 p$ & pyrrolidin -1-yl & $\mathrm{N}\left(\mathrm{CH}_{3}\right) \mathrm{Ph}$ \\
\hline
\end{tabular}

approach differs from other pharmacokinetic prediction tools used in the field, such as PopPK modelling, which is also a compartment-based pharmacokinetic modelling [51]. However, in contrast to PBPK, in PopPK, the compartments are not linked to anatomic or physiologic values. In general, PopPK basically considers available pharmacokinetic information and builds a model that fits the data. PopPK modelling follows a "top-down" approach, being mainly empirical and starting with a very simple model (usually one compartment) and assessing linear kinetics. Additional peripheral compartments can then be added to interpret the data and assess statistical significance. Differently, PBPK models are based on the "bottom-up" approach, starting from the organ or at the tissue level [52]. In PBPK, simulated version of drug concentrations in various tissues versus time profiles is usually generated to carry out the analysis [53]. Using the PK-Sim/ Mobi platform (Bayer Technology Services), we have processed the 43 compounds composing library through PBPK to evaluate the plasma concentration-time profile (Figs. S1-S5, Supporting Information). Pharmacokinetic parameters used in our model, including area under the curve (AUC), maximum concentration (Cmax), time to reach maximum concentration (tmax), mean residence time (MRT), clearance (CL/F) and volume of distribution (Vd), are presented in Table S7. Overall, the PBPK analysis highlights compounds $\mathbf{1 a}, \mathbf{1 q}$ (with pyrimido[4,5-d] pyridazine-4,8-dione scaffold) and $\mathbf{3 b}$ (with pyrido(1,2-a) pyrimidine scaffold) displayed the highest values of AUC, followed by $\mathbf{1} \mathbf{k}$ and 1f. The respective Cmax values of these five terms were also higher than the Cmax values of the rest of the compounds (Table S8, Supporting Information). However, the value of absolute bioavailability results reduced to $18.5 \%$ for $\mathbf{1 q}, \mathbf{1} \mathbf{k}$ and $\mathbf{1 f}$ when the AUC after 
Table 4 Calculated V values used to rank binding affinity between ligands and proteins, for the selected proteins with $\delta \geq 1$

\begin{tabular}{|c|c|c|c|c|c|c|c|c|c|c|c|c|c|c|c|c|c|}
\hline \multirow[b]{2}{*}{ Ligand } & \multicolumn{17}{|c|}{ Protein (PDB code) } \\
\hline & $1 \times k k$ & $2 \mathrm{fb8}$ & 2rku & $2 x a b$ & 3131 & $3 \mathrm{lbz}$ & 3pe1 & $3 z x z$ & 4ase & $4 \mathrm{ks8}$ & $4 p 5 e$ & $\mathbf{4 q 0 7}$ & $\mathbf{4 q m z}$ & $4 \mathbf{u} \mathbf{j}$ & 4ual & $4 y c 8$ & $5 i 9 y$ \\
\hline Co-crystallised & 0.98 & 1.05 & 0.85 & 1.02 & 0.95 & 0.72 & 1.12 & 1.09 & 0.93 & 0.90 & 0.87 & 0.91 & 0.94 & 0.81 & 1.04 & 1.03 & 1.03 \\
\hline 1a & 1.04 & 1.03 & 1.06 & 0.95 & 1.02 & 0.77 & 1.02 & 0.93 & 0.85 & 0.99 & 0.89 & 1.04 & 0.90 & 0.96 & 1.02 & 1.00 & 1.06 \\
\hline $1 b$ & 1.07 & 1.06 & 1.03 & 0.94 & 1.09 & 0.77 & 1.18 & 1.02 & 0.93 & 1.03 & 0.96 & 0.97 & 0.95 & 1.05 & 1.19 & 0.99 & 0.99 \\
\hline 1c & 1.08 & 1.05 & 1.01 & 0.90 & 0.97 & 0.77 & 1.13 & 0.99 & 0.90 & 1.02 & 0.93 & 0.91 & 0.94 & 1.02 & 1.12 & 1.03 & 0.96 \\
\hline 1d & 1.09 & 1.09 & 1.14 & 0.87 & 1.03 & 0.82 & 1.17 & 1.07 & 0.94 & 1.03 & 1.02 & 0.91 & 1.01 & 1.01 & 1.06 & 1.08 & 0.99 \\
\hline $1 \mathrm{e}$ & 0.96 & 1.03 & 1.00 & 0.95 & 1.02 & 0.73 & 1.05 & 0.97 & 0.86 & 0.94 & 0.94 & 1.02 & 0.92 & 0.99 & 0.98 & 0.95 & 0.96 \\
\hline 1f & 0.96 & 1.06 & 0.96 & 0.96 & 1.03 & 0.72 & 1.01 & 1.08 & 0.85 & 0.94 & 0.98 & 1.03 & 0.96 & 0.99 & 0.97 & 0.91 & 0.94 \\
\hline $1 \mathrm{~g}$ & 1.10 & 1.10 & 1.06 & 0.99 & 1.10 & 0.78 & 1.13 & 1.01 & 0.95 & 1.06 & 1.01 & 1.00 & 0.99 & 1.05 & 1.10 & 1.07 & 1.01 \\
\hline $1 \mathrm{~h}$ & 1.04 & 1.03 & 1.11 & 1.02 & 1.04 & 0.75 & 1.01 & 0.99 & 0.88 & 1.05 & 0.99 & 1.00 & 0.97 & 1.00 & 0.97 & 0.99 & 1.05 \\
\hline $1 \mathbf{i}$ & 0.95 & 1.04 & 1.08 & 1.02 & 1.11 & 0.75 & 1.01 & 1.06 & 0.89 & 0.97 & 0.94 & 1.00 & 1.02 & 1.06 & 1.08 & 0.98 & 0.98 \\
\hline $1 \mathrm{j}$ & 1.04 & 0.99 & 1.08 & 0.99 & 1.05 & 0.77 & 0.97 & 0.98 & 0.93 & 0.99 & 1.06 & 0.97 & 1.01 & 1.02 & 1.06 & 0.96 & 1.00 \\
\hline $1 k$ & 1.01 & 1.07 & 0.96 & 0.93 & 1.02 & 0.75 & 1.08 & 0.90 & 0.83 & 0.95 & 0.93 & 1.06 & 0.91 & 0.94 & 1.01 & 0.99 & 0.98 \\
\hline 11 & 1.09 & 1.15 & 1.06 & 0.96 & 1.07 & 0.74 & 1.07 & 0.92 & 0.89 & 1.03 & 0.96 & 0.99 & 0.96 & 1.04 & 1.16 & 0.96 & 1.01 \\
\hline $1 \mathrm{~m}$ & 1.07 & 1.08 & 1.09 & 1.04 & 1.07 & 0.80 & 1.15 & 1.05 & 0.96 & 1.07 & 0.99 & 0.97 & 0.99 & 1.02 & 1.08 & 1.03 & 1.01 \\
\hline 1n & 1.04 & 1.07 & 1.05 & 1.00 & 1.07 & 0.78 & 1.12 & 1.04 & 0.94 & 1.05 & 0.99 & 0.96 & 0.97 & 1.01 & 1.11 & 1.00 & 1.03 \\
\hline 10 & 0.95 & 1.02 & 1.05 & 0.99 & 1.01 & 0.72 & 1.08 & 1.08 & 0.91 & 0.97 & 0.99 & 1.00 & 0.96 & 1.05 & 1.07 & 0.96 & 0.98 \\
\hline $1 p$ & 1.11 & 1.08 & 1.06 & 0.99 & 1.08 & 0.78 & 1.12 & 1.01 & 0.95 & 1.05 & 1.00 & 1.01 & 0.96 & 1.03 & 1.10 & 0.98 & 0.99 \\
\hline $1 q$ & 0.93 & 1.12 & 0.94 & 0.93 & 1.00 & 0.71 & 1.01 & 1.05 & 0.83 & 0.91 & 0.95 & 1.05 & 0.95 & 0.99 & 1.01 & 0.91 & 0.93 \\
\hline $1 \mathbf{r}$ & 1.06 & 1.13 & 1.10 & 0.97 & 1.07 & 0.79 & 1.08 & 0.98 & 0.88 & 1.04 & 1.00 & 1.01 & 1.05 & 1.03 & 1.08 & 0.95 & 1.01 \\
\hline 1s & 1.02 & 1.11 & 1.02 & 0.94 & 0.99 & 0.74 & 0.91 & 1.00 & 0.86 & 0.93 & 0.93 & 0.97 & 0.98 & 0.97 & 1.01 & 1.04 & 1.05 \\
\hline $1 t$ & 1.11 & 1.11 & 1.06 & 0.97 & 1.09 & 0.79 & 1.08 & 0.97 & 0.98 & 1.02 & 0.93 & 0.96 & 0.98 & 1.03 & 1.14 & 1.01 & 1.02 \\
\hline $1 \mathbf{u}$ & 0.93 & 1.13 & 0.96 & 0.97 & 0.99 & 0.73 & 1.01 & 1.00 & 0.85 & 0.97 & 0.92 & 1.00 & 0.93 & 0.99 & 1.03 & 0.93 & 0.92 \\
\hline $2 a$ & 0.99 & 0.98 & 1.07 & 0.95 & 1.00 & 0.74 & 0.99 & 1.08 & 0.88 & 0.97 & 0.95 & 0.99 & 0.91 & 0.95 & 1.00 & 0.96 & 0.94 \\
\hline $2 b$ & 1.05 & 1.06 & 0.96 & 0.93 & 1.05 & 0.76 & 1.09 & 0.98 & 0.87 & 1.02 & 1.00 & 1.03 & 0.96 & 1.00 & 1.03 & 0.93 & 0.94 \\
\hline $2 c$ & 0.98 & 0.96 & 0.99 & 0.98 & 0.99 & 0.74 & 1.07 & 1.12 & 0.84 & 0.98 & 0.92 & 0.93 & 0.93 & 1.00 & 1.05 & 1.00 & 0.98 \\
\hline $2 d$ & 0.93 & 0.94 & 0.99 & 0.96 & 0.93 & 0.76 & 1.07 & 1.06 & 0.83 & 0.94 & 0.90 & 0.91 & 0.92 & 0.90 & 0.98 & 0.97 & 0.90 \\
\hline $2 e$ & 0.86 & 0.96 & 1.06 & 1.04 & 1.03 & 0.74 & 1.07 & 1.11 & 0.88 & 0.95 & 0.93 & 0.95 & 1.01 & 1.04 & 1.15 & 1.00 & 0.98 \\
\hline $2 f$ & 1.10 & 0.99 & 1.13 & 1.09 & 1.12 & 0.76 & 1.13 & 1.07 & 1.05 & 1.00 & 0.98 & 1.03 & 0.97 & 1.06 & 1.04 & 1.21 & 1.03 \\
\hline $2 \mathrm{~g}$ & 1.02 & 0.99 & 1.13 & 0.92 & 1.02 & 0.74 & 1.04 & 1.11 & 0.92 & 0.98 & 0.99 & 0.98 & 0.94 & 1.00 & 0.99 & 1.00 & 0.96 \\
\hline $2 h$ & 1.07 & 1.08 & 0.95 & 0.92 & 1.07 & 0.74 & 1.12 & 0.99 & 0.84 & 1.01 & 0.91 & 0.98 & 0.98 & 1.05 & 1.07 & 0.92 & 0.96 \\
\hline $2 \mathrm{i}$ & 0.96 & 0.96 & 0.98 & 0.95 & 1.03 & 0.76 & 0.96 & 1.01 & 1.00 & 0.90 & 0.86 & 0.98 & 0.99 & 1.04 & 0.98 & 1.06 & 1.06 \\
\hline $2 j$ & 1.15 & 1.14 & 1.15 & 0.96 & 1.10 & 0.80 & 1.07 & 1.08 & 1.02 & 0.98 & 0.96 & 0.92 & 0.99 & 1.07 & 1.10 & 1.16 & 0.99 \\
\hline $2 k$ & 1.15 & 1.08 & 1.15 & 0.95 & 1.02 & 0.79 & 0.96 & 1.14 & 0.99 & 1.01 & 0.93 & 0.95 & 1.03 & 1.08 & 1.14 & 1.14 & 1.07 \\
\hline 21 & 0.99 & 0.98 & 0.99 & 0.95 & 0.98 & 0.76 & 1.04 & 0.99 & 0.85 & 0.92 & 0.96 & 0.91 & 0.94 & 0.93 & 1.03 & 0.95 & 1.02 \\
\hline $2 \mathrm{~m}$ & 1.06 & 1.02 & 1.09 & 0.94 & 0.94 & 0.74 & 0.99 & 0.99 & 0.90 & 0.96 & 1.01 & 0.88 & 0.97 & 1.00 & 0.95 & 1.07 & 1.05 \\
\hline $2 n$ & 0.99 & 0.99 & 0.96 & 0.97 & 0.97 & 0.74 & 1.08 & 1.08 & 0.89 & 1.02 & 0.97 & 0.88 & 1.01 & 0.99 & 1.14 & 0.92 & 0.95 \\
\hline 20 & 1.00 & 1.00 & 0.97 & 1.01 & 0.99 & 0.78 & 0.98 & 1.08 & 0.88 & 0.95 & 0.99 & 0.91 & 0.98 & 1.08 & 1.13 & 0.97 & 0.96 \\
\hline $2 p$ & 0.96 & 1.06 & 0.98 & 0.99 & 0.88 & 0.80 & 1.10 & 1.21 & 0.96 & 1.03 & 1.00 & 0.91 & 1.04 & 1.03 & 1.04 & 1.09 & 1.10 \\
\hline $3 \mathbf{a}$ & 0.86 & 0.92 & 0.90 & 0.95 & 0.92 & 0.70 & 0.92 & 0.94 & 0.84 & 0.87 & 0.86 & 0.90 & 0.91 & 0.88 & 0.86 & 0.92 & 0.90 \\
\hline $3 b$ & 0.99 & 1.08 & 1.00 & 1.02 & 1.05 & 0.80 & 1.06 & 1.06 & 0.93 & 0.93 & 0.88 & 1.00 & 0.97 & 0.95 & 1.06 & 0.98 & 0.96 \\
\hline $3 c$ & 1.17 & 1.13 & 1.17 & 1.09 & 1.08 & 0.85 & 1.05 & 1.00 & 0.99 & 0.98 & 0.99 & 0.93 & 1.02 & 0.97 & 1.00 & 1.11 & 1.08 \\
\hline 3d & 1.06 & 1.12 & 1.15 & 1.11 & 1.11 & 0.87 & 1.08 & 1.06 & 0.96 & 0.99 & 1.01 & 1.04 & 1.01 & 1.03 & 1.13 & 1.08 & 1.03 \\
\hline $3 e$ & 1.03 & 1.14 & 1.06 & 1.08 & 1.20 & 0.77 & 1.04 & 1.09 & 0.99 & 1.00 & 0.98 & 0.96 & 0.99 & 0.94 & 1.08 & 1.00 & 1.06 \\
\hline 3f & 1.00 & 1.11 & 1.11 & 1.07 & 1.14 & 0.72 & 1.07 & 0.82 & 0.97 & 0.87 & 0.92 & 1.06 & 1.00 & 0.95 & 1.05 & 0.97 & 1.06 \\
\hline
\end{tabular}




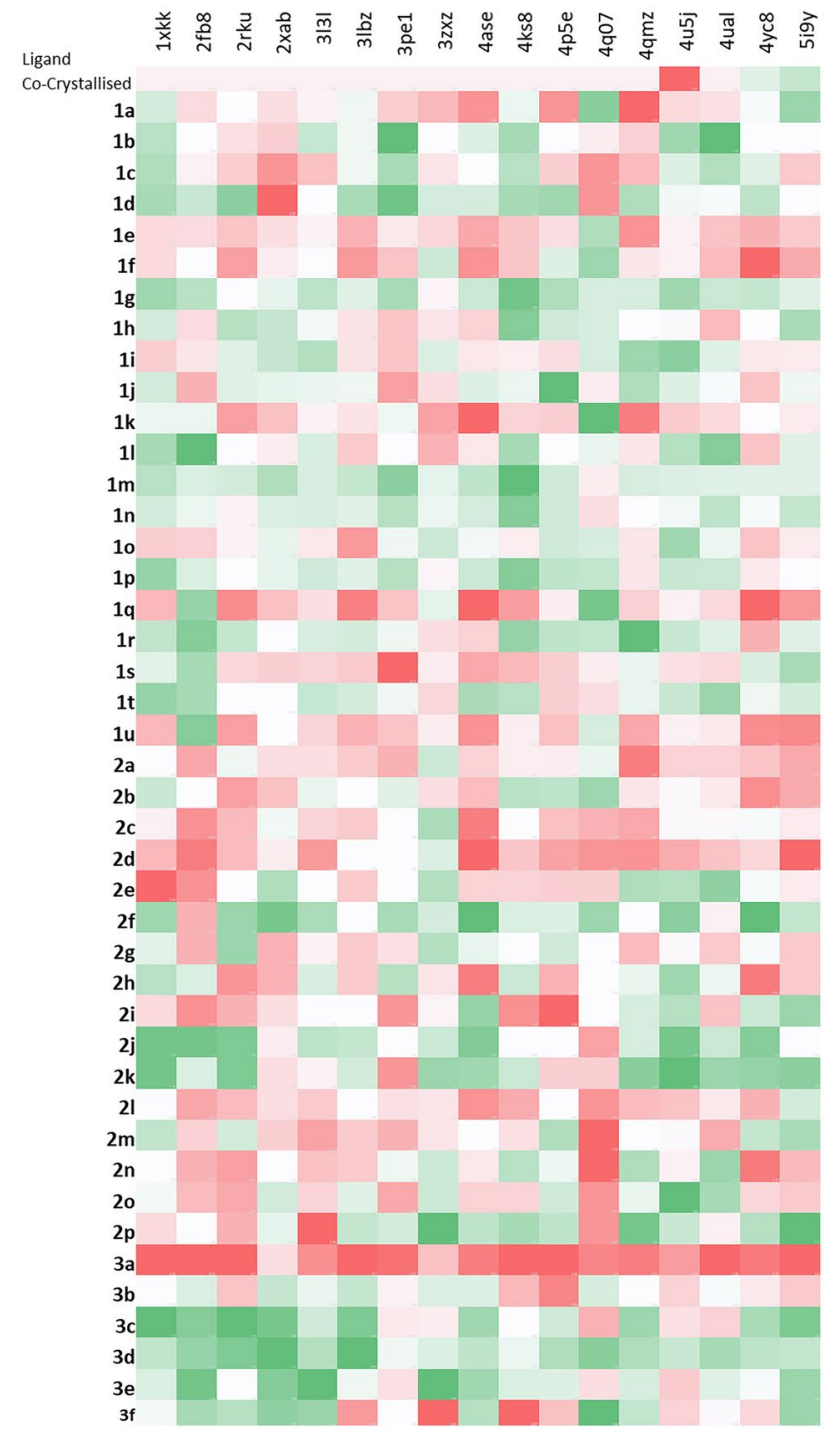

Fig. 4 Matrix of the calculated V values used to rank binding affinities between ligands and cellular targets, for selected proteins with $\delta \geq 1$. Green-high value, red-low value oral administration is taken into consideration, while $\mathbf{3 b}$ and 1a still possess a bioavailability of 44.6 and $36.3 \%$. Vd values for 3b, 1q and 1a are 337.7, 278.6 and $442.1 \mathrm{~mL} /$ $\mathrm{kg}$, respectively. These low values suggest that the reduced lipophilic nature of $\mathbf{3 b}, \mathbf{1 q}$ and $\mathbf{1 a}$ may be a determinant for their lower volume of distribution, influencing their lower absorption and bioavailability after oral administration. In this regard, a correlation coefficient of 0.4 was found (Fig. 5) for both cLogP (i.e. lipophilicity measure) versus $\mathrm{Vd}$, and $\mathrm{cLogP}$ versus bioavailability, in line with the assumption that the lipophilicity of the molecules may play a role in the absorption when administrated orally.

Among the terms with pyrimido[4,5-d]pyridazine4,8-dione scaffold, compound $\mathbf{1 t}$ showed the highest value of bioavailability $(95.2 \%)$ followed by compounds $\mathbf{1 i}$ and $1 \mathbf{1}$, having a bioavailability of 92.4 and $90.8 \%$, respectively (Table S9, Supporting Information). Therefore, these compounds demonstrate optimal features for oral administration. Compound $1 \mathrm{t}$ has a high $\operatorname{cog} \mathrm{P}$ (i.e. 2.18) due to the presence of the $\mathrm{CH}_{2} \mathrm{cC}_{6} \mathrm{H}_{11}$ residue at $\mathrm{R} 1$ and the ethyl group at $\mathrm{R} 3$ of the pyrimido[4,5-d]pyridazine-4,8-dione core, in addition to the $\mathrm{Ph}$ at $\mathrm{R}$ and the $\mathrm{H}$ at $\mathrm{R} 2$ which are common to the majority of the other compounds with the same scaffold (Table 1). Similarly, also compounds $1 \mathbf{i}$ and 11 possess relatively high values of cLogP (i.e. 1.866 and 1.9261 respectively), suggesting higher absorption in the case of oral formulations. With regard to the analogues with 1,8-naphthyridin-4-one scaffold, $\mathbf{2} \mathbf{g}$ and $\mathbf{2} \mathbf{h}$ (with 4- $\mathrm{Cl}-\mathrm{CH}_{2} \mathrm{Ph}$ and $3-\mathrm{F}-\mathrm{C}_{6} \mathrm{H}^{4}$ residues in the $\mathrm{X}$ position; Table 2) display the highest bioavailability (i.e. $98.1 \%$ and 98.5\%) within the library (Table S9, Supporting Information). The cLogP values of $\mathbf{2} \mathbf{h}$ and $\mathbf{2} \mathbf{g}$ are also high (i.e. 2.107 and 2.0004). For the analogues with pyrido(1,2-a) pyrimidine scaffold, compound 3d possess the highest bioavailability (i.e. 96.4\%; Table S9, Supporting Information), having a cLogP of 2.29 which is most likely determined by the presence of the $\mathrm{CH}(\mathrm{Ph})_{2}$ residue at $\mathrm{R} 1$ position (Table 3). However, also 3c and 3e have high cLogP and
Fig. 5 Scatter plots for the correlation between cLogP and PK parameters (Vd and bioavailability). a Correlation of R2 $=0.4$ between cLogP and $\mathrm{Vd}$. b Correlation of $\mathrm{R} 2=0.4$ between cLogP and bioavailability (\%)

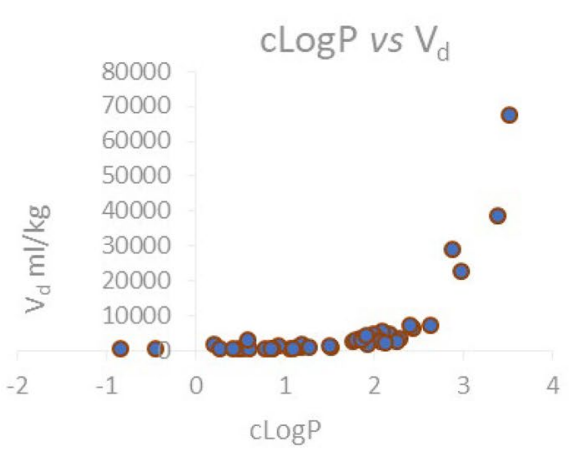

(a)

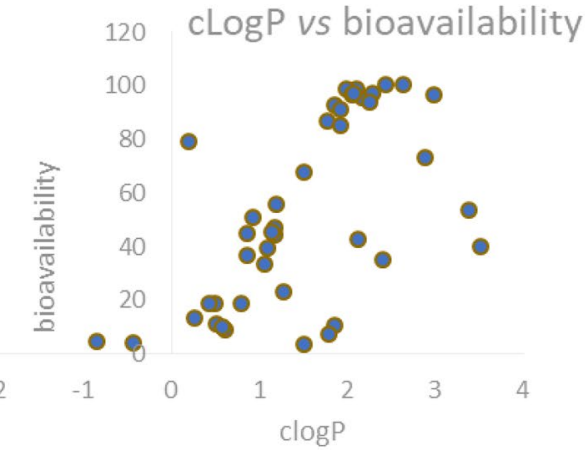

(b) 
bioavailability values in close range. Lastly, compounds $\mathbf{1 c}$, $\mathbf{1} \mathbf{~ m}, \mathbf{1 n}, \mathbf{2} \mathbf{~ m}$ and 3a, resulted in very low bioavailability values of 2.9, 8.3, 9.6, 3.1, 3.2\% (Table S9, Supporting Information), suggesting that these analogues would have poor absorption after oral administration.

\section{Conclusions}

In this study, a new set of scaffold-diverse compounds was developed by means of a two-step molecular design process based on scaffold hopping/bio-isosteric replacement and automated ligand growing methodologies. We have used this approach to carry out a preliminary evaluation of a compound dataset versus a panel of cellular proteins involved in cancer cell survival and cancer progression. Standard synthetic procedures allow the ease production of the molecular series used in this work, which are based on three bi-cyclic heterocycles, i.e. pyrimido[4,5-d]pyridazine4,8-dione, 1,8-napthyridin-4-one and pyrido(1,2-a)pyrimidine. Our data further confirm the advantages of inverse virtual screening protocols as an in silico approach to implement conventional methods in drug design and discovery, allowing the preliminary assessment of affinity between a set of ligands and suitable pharmacological targets. The normalisation of the binding energies proves valid to identify significant interactions between the ligands and several protein targets used in the study (i.e. HSP90, PARP, VEGFR2, mammalian sterile20-like kinase 3, EGFR kinase, B-Raf kinase, PLK1, protein kinase CK2, c-Met, PAK6 kinase, DNPH1, carbonic anhydrase IX, c-Src, MRCK beta, c-Abl kinase, and ephrin A2 receptor protein kinase). In parallel, the normalisation process also proposes nine ligands $(\mathbf{1 b}$, $1 \mathbf{k}, 1 \mathbf{1 q}, \mathbf{2 c}, \mathbf{2 j}, \mathbf{2 p}, \mathbf{3 a}, \mathbf{3 c}$ and $\mathbf{3 f}$ ) as useful tools for biomedical investigations in the context of cancer chemotherapy. With a bioavailability higher than $90 \%$, compounds $\mathbf{1 t}, \mathbf{2} \mathbf{~ g}$, $\mathbf{2} \mathbf{h}$ and $\mathbf{3 d}$ also demonstrate permeability and absorption features worth to be assessed in subsequent pharmacological studies.

Supplementary information The online version contains supplementary material available at https://doi.org/10.1007/s11224-022-01889-0.

Acknowledgements Andrew Beavil (King's College London) is gratefully acknowledged for providing software resources via King's Virtual Desktop.

Author contribution Conceptualisation, G.F., L.C. and A.C. Software, G.F and S.N. In silico methodology and experimental work, A.B., G.F. and S.N. Synthetic methodology and experimental work, C.B. and N.C. Data analysis, C.B., N.C., L.C., C.V., A.B., G.F. and S.N. Data curation, L.C., C.V., G.F. and S.N. Writing-original draft preparation, L.C and G.F. Writing - review and editing, M.P. and A.C. Supervision and project administration, M.P and A.C.
Funding S.N. was funded by the Post-Doc Fellowships Programme (3-1/PDFP/HEC/2020/856), Higher Education Commission, Pakistan. G.F. is currently a Marie Skłodowska-Curie fellow funded by the European Union's Horizon 2020 Research and Innovation Programme under grant agreement No. 893784.

Availability of data and material The online version contains supplementary material and data available at https:

\section{Declarations}

Conflict of interest The authors declare no competing interests.

Open Access This article is licensed under a Creative Commons Attribution 4.0 International License, which permits use, sharing, adaptation, distribution and reproduction in any medium or format, as long as you give appropriate credit to the original author(s) and the source, provide a link to the Creative Commons licence, and indicate if changes were made. The images or other third party material in this article are included in the article's Creative Commons licence, unless indicated otherwise in a credit line to the material. If material is not included in the article's Creative Commons licence and your intended use is not permitted by statutory regulation or exceeds the permitted use, you will need to obtain permission directly from the copyright holder. To view a copy of this licence, visit http://creativecommons.org/licenses/by/4.0/.

\section{References}

1. Dolle RE (2004) Comprehensive survey of combinatorial library synthesis: 2003. J Comb Chem 6:623-679. https://doi.org/10. $1021 / \mathrm{cc} 0499082$

2. Kaushik NK, Kaushik N, Attri P, Kumar N, Kim CH, Verma AK, Choi EH (2013) Biomedical importance of indoles. Molecules 18:6620-6662. https://doi.org/10.3390/molecules18066620

3. Macarron R, Banks MN, Bojanic D, Burns DJ, Cirovic DA, Garyantes T, Green DVS, Hertzberg RP, Janzen WP, Paslay JW et al (2011) Impact of high-throughput screening in biomedical research. Nat Rev Drug Discov 10:188-195. https://doi.org/10. 1038/nrd3368

4. Shi F, Zeng XN, Cao XD, Zhang S, Jiang B, Zheng WF, Tu SJ (2012) Design and diversity-oriented synthesis of novel 1,4-thiazepan-3-ones fused with bioactive heterocyclic skeletons and evaluation of their antioxidant and cytotoxic activities. Bioorg Med Chem Lett 22:743746. https://doi.org/10.1016/j.bmcl.2011.09.081

5. Isidro-Llobet A, Murillo T, Bello P, Cilibrizzi A, Hodgkinson JT, Galloway WRJD, Bender A, Welch M, Spring DR (2011) Diversity-oriented synthesis of macrocyclic peptidomimetics. PNAS 108:6793. https://doi.org/10.1073/pnas.1015267108

6. Lipinski C, Hopkins A (2004) Navigating chemical space for biology and medicine. Nature 432:855-861. https://doi.org/10.1038/ nature 03193

7. Sauer WHB, Schwarz MK (2003) Size doesn't matter: scaffold diversity, shape diversity and biological activity of combinatorial libraries. Chimia 57:276-283. https://doi.org/10.2533/ 000942903777679253

8. Sauer WHB, Schwarz MK (2003) Molecular shape diversity of combinatorial libraries: a prerequisite for broad bioactivity. $\mathrm{J}$ Chem Inform Comput Sci 43:987-1003. https://doi.org/10.1021/ ci025599w 
9. Spandl RJ, Bender A, Spring DR (2008) Diversity-oriented synthesis; a spectrum of approaches and results. Org Biomol Chem 6:1149-1158. https://doi.org/10.1039/B719372F

10. Burke MD, Berger EM, Schreiber SL (2003) Generating diverse skeletons of small molecules combinatorially. Science 302:613618. https://doi.org/10.1126/science.1089946

11. Kennedy JP, Williams L, Bridges TM, Daniels RN, Weaver D, Lindsley CW (2008) Application of combinatorial chemistry science on modern drug discovery. J Comb Chem 10:345-354. https://doi.org/10.1021/cc700187t

12. Shelat AA, Guy RK (2007) Scaffold composition and biological relevance of screening libraries. Nat Chem Bio 3:442-446. https:// doi.org/10.1038/nchembio0807-442

13. Austin ND, Sahinidis NV, Trahan DW (2016) Computer-aided molecular design: an introduction and review of tools, applications, and solution techniques. Chem Eng Res Des 116:2-26. https://doi.org/10.1016/j.cherd.2016.10.014

14. Floresta G, Pittalà V, Sorrenti V, Romeo G, Salerno L, Rescifina A (2018) Development of new HO-1 inhibitors by a thorough scaffold-hopping analysis. Bioorg Chem 81:334-339. https://doi. org/10.1016/j.bioorg.2018.08.023

15. Lavecchia A, Di Giovanni C (2013) Virtual screening strategies in drug discovery: a critical review. Curr Med Chem 20:2839-2860. https://doi.org/10.2174/09298673113209990001

16. Ruiz-Torres V, Encinar JA, Herranz-López M, Pérez-Sánchez A, Galiano V, Barrajón-Catalán E, Micol V (2017) An updated review on marine anticancer compounds: the use of virtual screening for the discovery of small-molecule cancer drugs. Molecules 22:1037. https://doi.org/10.3390/molecules22071037

17. Cilibrizzi A, Floresta G, Abbate V, Giovannoni MP (2019) iVS analysis to evaluate the impact of scaffold diversity in the binding to cellular targets relevant in cancer. J Enzyme Inhib Med Chem 34:44-50. https://doi.org/10.1080/14756366.2018.1518960

18. Floresta G, Crocetti L, Giovannoni MP, Biagini P, Cilibrizzi A (2020) Repurposing strategies on pyridazinone-based series by pharmacophore- and structure-driven screening. J Enzyme Inhib Med Chem 35:1137-1144. https://doi.org/10.1080/14756366. 2020.1760261

19. Xu X, Huang M, Zou X (2018) Docking-based inverse virtual screening: Methods, applications, and challenges. Biophys Rep 4:1-16. https://doi.org/10.1007/s41048-017-0045-8

20. Lauro G, Masullo M, Piacente S, Riccio R, Bifulco G (2012) Inverse virtual screening allows the discovery of the biological activity of natural compounds. Bioorg Med Chem 20:3596-3602. https://doi.org/10.1016/j.bmc.2012.03.072

21. Lauro G, Romano A, Riccio R, Bifulco G (2011) Inverse virtual screening of antitumor targets: Pilot study on a small database of natural bioactive compounds. J Nat Prod 74:1401-1407. https:// doi.org/10.1021/np100935s

22. Dal Piaz V, Aguilar IN, Buil AMA, Garrido RY, Giovannoni MP, Gracia FJ (2005) Pyridazin-3-(2H)-one derivatives and their use as PDE4 inhibitors. PCT WO2005049581

23. Hirose T, Mishio S, Matsumoto J, Minami S (1982) Pyridonecarboxylic acids as antibacterial agents. I. Synthesis and antibacterial activity of 1-alkyl-1,4-dihydro-4-oxo-1,8- and 1,6naphthyridine-3-carboxylic acids. Chem Pharm Bull 30:23992409. https://doi.org/10.1248/cpb.30.2399

24. Shur M, Israelstam SS (1968) The reaction of aminoheterocycles with reactive esters. I. Aminopyridines. J Org Chem 33:30153020. https://doi.org/10.1021/jo01272a002

25. Hermecz I, Vasvari-Debreczy L, Horvath A, Sipos J, Balogh M, Podanyi B, Kovacs K (1998) Nitrogen bridgehead compounds. Part 92. ACH - Models Chem 135:515-528

26. Floresta G, Cilibrizzi A, Abbate V, Spampinato A, Zagni C, Rescifina A (2019) 3D-QSAR assisted identification of FABP4 inhibitors: an effective scaffold hopping analysis/QSAR evaluation.
Bioorg Chem 84:276-284. https://doi.org/10.1016/j.bioorg.2018. 11.045

27. Floresta G, Cilibrizzi A, Abbate V, Spampinato A, Zagni C, Rescifina A (2019) FABP4 inhibitors 3D-QSAR model and isosteric replacement of BMS309403 datasets. Data Brief 22:471-483. https://doi. org/10.1016/j.dib.2018.12.047

28. Encinas LA (2016) Chapter 6 quinolines: privileged scaffolds in medicinal chemistry. Privileged Scaffolds in Medicinal Chemistry: Design Synthesis, Evaluation. The Royal Society of Chemistry, pp 132-146

29. Singh S, Kaur G, Mangla V, Gupta MK (2015) Quinoline and quinolones: promising scaffolds for future antimycobacterial agents. J Enzyme Inhib Med Chem 30:492-504. https://doi.org/10.3109/ 14756366.2014.930454

30. Floresta G, Amata E, Gentile D, Romeo G, Marrazzo A, Pittalà V, Salerno L, Rescifina A (2019) Fourfold filtered statistical/computational approach for the identification of imidazole compounds as HO-1 inhibitors from natural products. Mar Drugs 17:113. https:// doi.org/10.3390/md17020113

31. Floresta G, Gentile D, Perrini G, Patamia V, Rescifina A (2019) Computational tools in the discovery of FABP4 ligands: a statistical and molecular modeling approach. Mar Drugs 17:624. https:// doi.org/10.3390/md17110624

32. Cheeseright T, Mackey M, Rose S, Vinter A (2006) Molecular field extrema as descriptors of biological activity: Definition and validation. J Chem Info Model 46:665-676. https://doi.org/10. 1021/ci050357s

33. Rogers D, Hahn M (2010) Extended-connectivity fingerprints. J Chem Inf Model 50:742-754. https://doi.org/10.1021/ci100050t

34. Renzi G (1969) Isoxazolo[3,4-d]pyridazin-7-one derivatives. Il Farmaco 24:885-892

35. Renzi G, Dal Piaz V, Musante C (1968) Pyridazin-3-(2H)-one derivatives and their use as PDE4 inhibitors. Gazz Chim Ital 98:656-666

36. Hermecz I, Mészáros Z, Vasvári-Debreczy L, Horváth Á, Horváth G, Pongor-Csákvári M (1977) Nitrogen bridgehead compounds. Part 4. $1 \rightarrow 3 \mathrm{~N} \rightarrow$ C-acyl migration. Part 2. J Chem Soc Perkin Trans I:789-795. https://doi.org/10.1039/P19770000789

37. Lappin GR (1948) Cyclization of 2-aminopyridine derivatives. I. Substituted Ethyl 2-Pyridylaminomethylenemalonates 1,2. J Am Chem Soc 70:3348-3350. https://doi.org/10.1021/ja01190a038

38. Lesher GY, Froelich EJ, Gruett MD, Bailey JH, Brundage RP (1962) 1,8-naphthyridine derivatives. A new class of chemotherapeutic agentS. J Med Pharm Chem 5:1063-1065. https://doi.org/ 10.1021/jm01240a021

39. Matsumoto J, Miyamoto T, Minamida A, Nishimura Y, Egawa H, Nishimura H (1984) 1,4-Dihydro-4-oxopyridinecarboxylic acids as antibacterial agents. 2. Synthesis and structure-activity relationships of 1,6,7-trisubstituted 1,4-dihydro-4-oxo-1,8-naphthyridine3 -carboxylic acids, including enoxacin, a new antibacterial agent. J Med Chem 27:292-301. https://doi.org/10.1021/jm00369a011

40. Mishio S, Hirose T, Minamida A, Matsumoto J, Minami S (1985) Pyridonecarboxylic acids as antibacterial agents. V. Synthesis of 1-vinyl-1,4-dihydro-4-oxo-1,8- and 1,6-naphthyridine-3-carboxylic acids. Chem Pharm Bull 33:4402-4408. https://doi.org/10.1248/ cpb.33.4402

41. Agui H, Saji I, Nakashita M (1979) 1-Substituted-1,4-dihydro4-oxo-3-pyridinecarboxylic acid derivatives. Jpn. Kokai Tokkyo Koho. JP 54112877 A 19790904

42. Abad-Zapatero C, Metz JT (2005) Ligand efficiency indices as guideposts for drug discovery. Drug Discov Today 10:464-469. https://doi.org/10.1016/s1359-6446(05)03386-6

43. Giovannoni MP, Ciciani G, Cilibrizzi A, Crocetti L, Daniele S, Di Cesare ML, Ghelardini C, Giacomelli C, Guerrini G, Martini C et al (2015) Further studies on pyrazolo[1',5':1,6]pyrimido[4,5-d] pyridazin- $4(3 \mathrm{H})$-ones as potent and selective human $\mathrm{A} 1$ adenosine 
receptor antagonists. Eur J Med Chem 89:32-41. https://doi.org/ 10.1016/j.ejmech.2014.10.020

44. Giovannoni MP, Vergelli C, Cilibrizzi A, Crocetti L, Biancalani C, Graziano A, Dal Piaz V, Loza MI, Cadavid MI, Díaz JL et al (2010) Pyrazolo[1',5':1,6]pyrimido[4,5-d]pyridazin-4(3H)-ones as selective human $\mathrm{A}(1)$ adenosine receptor ligands. Bioorg Med Chem 18:7890-7899. https://doi.org/10.1016/j.bmc.2010.09.043

45. Ahmed MF, Santali EY, Mohi El-Deen EM, Naguib IA, El-Haggar $R$ (2021) Development of pyridazine derivatives as potential EGFR inhibitors and apoptosis inducers: design, synthesis, anticancer evaluation, and molecular modeling studies. Bioorg Chem 106:104473. https://doi.org/10.1016/j.bioorg.2020.104473

46. Biagini P, Biancalani C, Graziano A, Cesari N, Giovannoni MP, Cilibrizzi A, Dal Piaz V, Vergelli C, Crocetti L, Delcanale M et al (2010) Functionalized pyrazoles and pyrazolo[3,4-d]pyridazinones: Synthesis and evaluation of their phosphodiesterase 4 inhibitory activity. Bioorg Med Chem 18:3506-3517. https://doi. org/10.1016/j.bmc.2010.03.066

47. He ZX, Gong YP, Zhang X, Ma LY, Zhao W (2021) Pyridazine as a privileged structure: an updated review on anticancer activity of pyridazine containing bioactive molecules. Eur J Med Chem 209:112946. https://doi.org/10.1016/j.ejmech.2020.112946

48. Newhouse BJ, Wenglowsky S, Grina J, Laird ER, Voegtli WC, Ren L, Ahrendt K, Buckmelter A, Gloor SL, Klopfenstein N et al (2013) Imidazo[4,5-b]pyridine inhibitors of B-Raf kinase. Bioorg
Med Chem Lett 23:5896-5899. https://doi.org/10.1016/j.bmcl. 2013.08.086

49. Montoir D, Barillé-Nion S, Tonnerre A, Juin P, Duflos M, Bazin MA (2016) Novel 1,6-naphthyridin-2(1H)-ones as potential anticancer agents targeting Hsp90. Eur J Med Chem 119:17-33. https://doi.org/10.1016/j.ejmech.2016.04.050

50. Poulin P, Theil F-P (2002) Prediction of pharmacokinetics prior to In Vivo studies. II. Generic physiologically based pharmacokinetic models of drug disposition. J Pharm Sci 91:1358-1370. https://doi.org/10.1002/jps.10128

51. Mould D, Upton R (2013) Basic concepts in population modeling, simulation, and model-based drug development-part 2: Introduction to pharmacokinetic modeling methods. CPT: Pharmacomet Syst Pharmacol 2:38. https://doi.org/10.1038/psp.2013.14

52. Gerlowski LE, Jain RK (1983) Physiologically based pharmacokinetic modeling: Principles and applications. J Pharm Sci 72:1103-1127. https://doi.org/10.1002/jps.2600721003

53. Kuepfer L, Niederalt C, Wendl T, Schlender J-F, Willmann S, Lippert J, Block M, Eissing T, Teutonico D (2016) Applied concepts in PBPK modeling: how to build a PBPK/PD model. CPT: Pharmacomet Syst Pharmacol 5:516-531. https://doi.org/10.1002/ psp4.12134

Publisher's Note Springer Nature remains neutral with regard to jurisdictional claims in published maps and institutional affiliations. 